ANÁLISIS TERMOFLUIDO Y NEUTRÓNICO

DE UN REACTOR NUCLEAR RÁPIDO

ENFRIADO CON PLOMO

Tesis que presenta

Alejandría Denisse Pérez Valseca

Para obtener el grado de

Maestro en Ciencias (Energía y Medio Ambiente) 



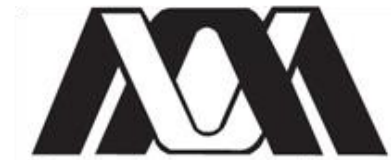

Casa abierta al tiempo UNIVERSIDAD AUTÓNOMA METROPOLITANA
UNIVERSIDAD AUTÓNOMA METROPOLITANA

UNIDAD IZTAPALAPA

POSGRADO EN ENERGÍA Y MEDIO AMBIENTE

\title{
ANÁLISIS TERMOFLUIDO Y NEUTRÓNICO DE UN REACTOR NUCLEAR RÁPIDO ENFRIADO CON PLOMO
}

\author{
Tesis que para obtener el grado de \\ MAESTRO EN CIENCIAS \\ (ENERGÍA Y MEDIO AMBIENTE)
}

Presenta:

Alejandría Denisse Pérez Valseca

Director:

Dr. Gilberto Espinosa-Paredes 


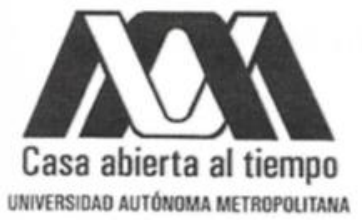

\author{
UNIVERSIDAD AUTÓNOMA METROPOLITANA \\ UNIDAD IZTAPALAPA \\ POSGRADO EN ENERGÍA Y MEDIO AMBIENTE
}

\title{
ANÁLISIS TERMOFLUIDO Y NEUTRÓNICO DE UN REACTOR NUCLEAR RÁPIDO ENFRIADO CON PLOMO
}

Tesis que para obtener el grado de

MAESTRO EN CIENCIAS

(ENERGÍA Y MEDIO AMBIENTE)

\section{Presenta:}

Alejandría Denisse Pérez Valseca

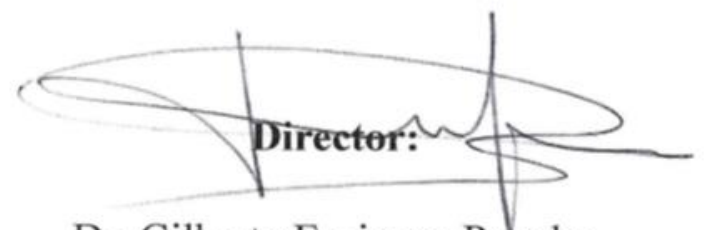

Dr. Gilberto Espinosa-Paredes 


\section{Agradecimientos}

La Maestría en Ciencias (Energía y Medio Ambiente) de la Universidad Autónoma Metropolitana Unidad Iztapalapa, pertenece al Padrón Nacional de Posgrados de Calidad del CONACyT y cuenta con apoyo del mismo Consejo con el convenio 003893; por lo que agradece la beca recibida, a través del convenio No. $\mathbf{4 0 4 8 9 0}$ para el desarrollo de esta tesis.

La autora agradece el financiamiento recibido del Consejo Nacional de Ciencia y Tecnología (CONACyT) y a la Secretaría de Energía (SENER) quienes financian el desarrollo de la plataforma AZTLAN, mediante el fondo sectorial CONACYT-SENER-SUSTENTABILIDAD ENERGÉTICA a través del proyecto No. $\mathbf{2 1 2 6 0 2 .}$ 


\section{Agradecimientos Personales}

Gracias,

A mis padres, Daniel y Adriana, por su amor y las enseñanzas puestas en mi corazón.

A Clemente, por su comprensión, su apoyo y por caminar junto a mí hacia mis sueños, pero sobre todo por su amor.

A mis hermanos, Daniela y Pedro, por su alegría y los ánimos que siempre me dan.

A Luis y Alejandra por su apoyo.

Al Dr. Gilberto, por la gran oportunidad de trabajar y aprender de él, por su forma de guiarme en el trabajo y por proporcionarme todos los recursos necesarios para la elaboración de este trabajo.

A los profesores de la comisión examinadora; Dra. Cecilia Martín del Campo, Dr. Rodolfo Vázquez y Dr. Armando Gómez, por sus oportunas observaciones y recomendaciones.

Al Fis. Alejandro Vázquez y al Dr. Sergio Quezada por su orientación.

A mis compañeros de la Tercera Generación del PEMA por el tiempo compartido, en especial a Juan Carlos y a César por la amistad y confianza que me regalaron.

A mis compañeros del Laboratorio de Simulación de Procesos Energéticos; Heriberto, Ricardo y Javier. 


\section{Comité Tutoral}

Director:

Dr. Gilberto Espinosa Paredes. Departamento de Ingeniería de Procesos e Hidráulica. División de Ciencias Básicas e Ingeniería. Universidad Autónoma Metropolitana Unidad Iztapalapa. 
El jurado designado por la

Comisión Académica del Posgrado en Energía y Medio Ambiente

de la Unidad Iztapalapa, aprobó la tesis que presentó

El día 28 de febrero del año 2017

Miembros del Jurado

Dra. Cecilia Martín del Campo Márquez

Presidente

Dr. Rodolfo Vázquez Rodríguez

Secretario

Dr. Armando Gómez Torres

Vocal 
El jurado designado por la

Comisión Académica del Posgrado en Energía y Medio Ambiente de la Unidad Iztapalapa, aprobó la tesis que presentó

El día 28 de febrero del año $\mathbf{2 0 1 7}$

Miembros del Jurado

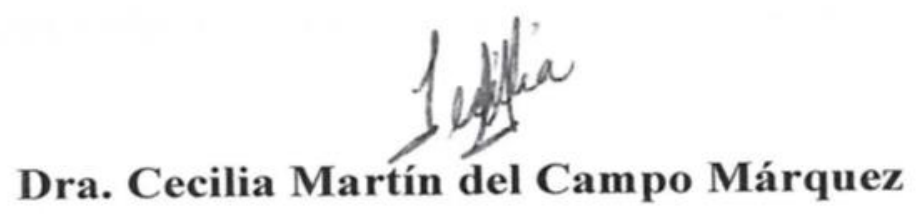

Presidente

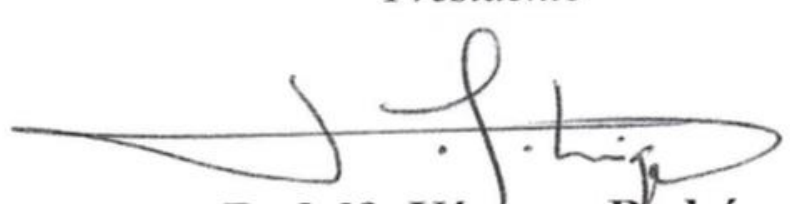

Dr. Rodolfo Vázquez Rlodríguez

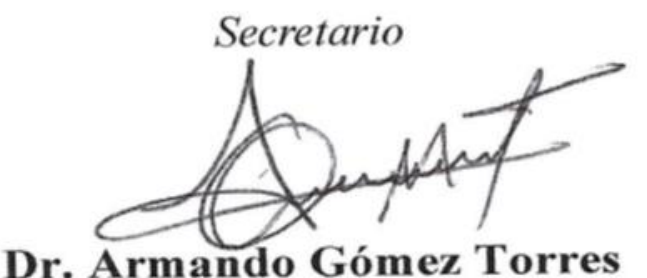

Vocal 


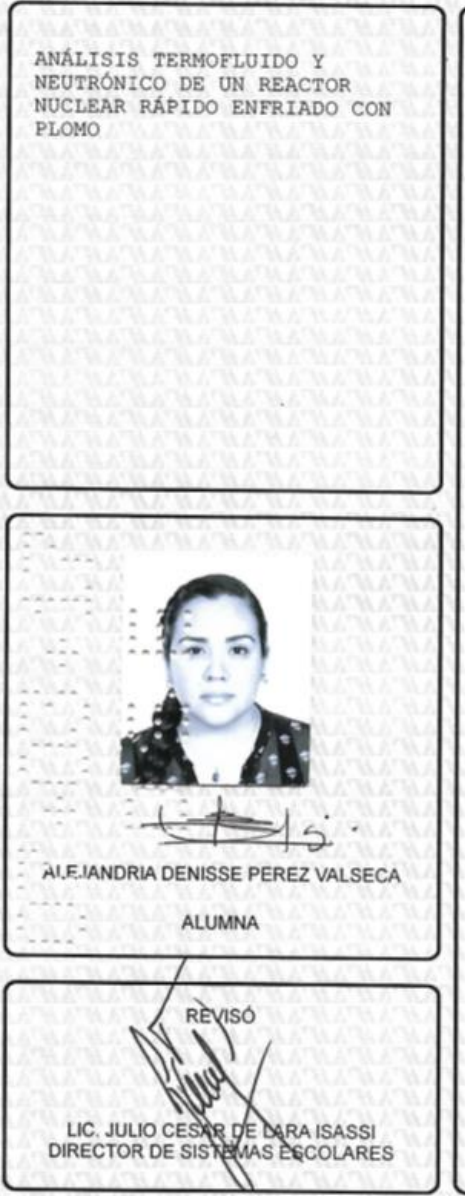

ANÁLISIS TERMOFLUIDO Y

UTRONICO DE UN REAC

En la Ciudad de México, se presentaron a 1as 16:00 horas del dia 28 del mes de febrero del año 2017 en la Unidad ztapalapa de la Universidad Autónoma Metropolitana, los suscritos miembros del jurado:

DRA. CECILIA MARTIN DEL CAMPO MARQUEZ

DR. ARMANDO MIGUEL GOMEZ TORRES

DODLER Z RODRIGZ

Bajo la Presidencia de la primera y con carácter de Secretario el último, se reunieron para proceder al Examen de Grado cuya denominacion aparece al margen, para la obtencion del grado de:

MAESTRA EN CIENCIAS (ENERGIA Y MEDIO AMBIENTE)

DE: ALEJANDRIA DENISSE PEREZ VALSECA

$y$ de acuerdo con el articulo 78 fraccion III del Reglamento de Estudios Superiores de la Universidad Autónoma Metropolitana, los miembros del jurado resolvieron:

\section{APROBAZ}

Acto continuo, la presidenta del jurado comunico a la interesada el resultado de la evaluacion $y$, en caso aprobatorio, le fue tomada la protesta.

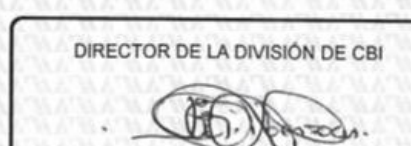

DR. JOSE GILBERTO CORDOBA HERRERA
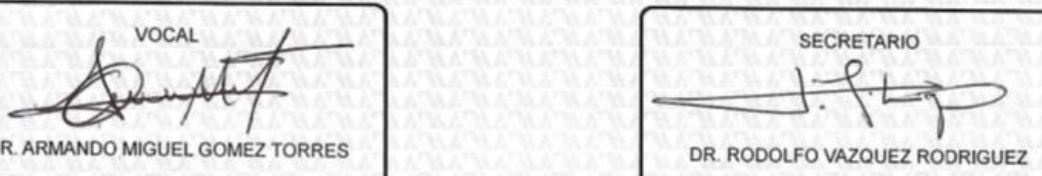

DR. ARMANDO MIGUEL GOMEZTORRES 


\section{Índice general}

Resumen..................................................................................................................................................................... 1

Abstract................................................................................................................................................................

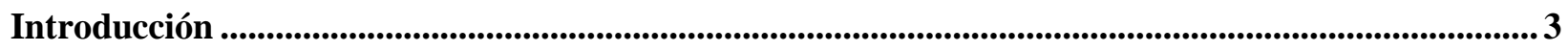

1. Antecedentes.............................................................................................................................................................

1.1 Situación energética mundial...........................................................................................................................5

1.2 Evolución de los Reactores Nucleares ..........................................................................................................

1.3 Reactores Nucleares de Generación IV....................................................................................................9

1.4. Los reactores nucleares y el desarrollo sustentable ..........................................................................18

2. Hipótesis y objetivos ................................................................................................................................................ 19

2.1. Hipótesis............................................................................................................................................................ 19

2.2. Objetivo general ............................................................................................................................................. 19

2.3. Objetivos específicos ..................................................................................................................................... 19

3. Reactor rápido enfriado con plomo..................................................................................................................21

3.1. Conceptos básicos de un LFR .........................................................................................................22

3.2. Descripción del reactor ALFRED ........................................................................................................25

4. Propiedades físicas ..................................................................................................................................................29

4.1. Propiedades del combustible, gap y encamisado..................................................................................29

4.2. Propiedades del plomo.......................................................................................................................................31

5. Análisis del reactor .....................................................................................................................................................39

5.1. Análisis de los procesos neutrónicos...................................................................................................39

5.1.1. Modelo puntual de la Cinética Neutrónica............................................................................. 39

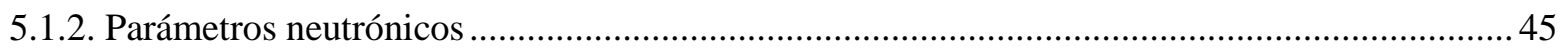

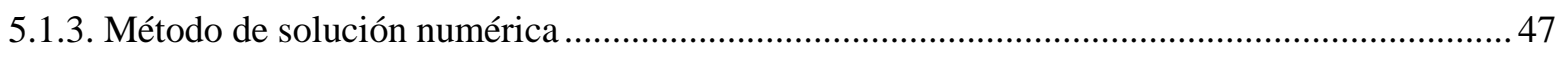

5.2. Análisis de transferencia de calor en la barra de combustible ........................................................ 48

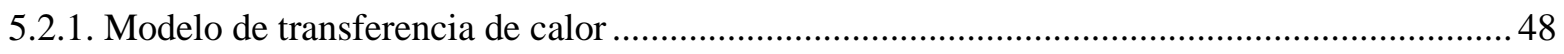

5.2.2. Parámetros de transferencia de calor................................................................................. 52 


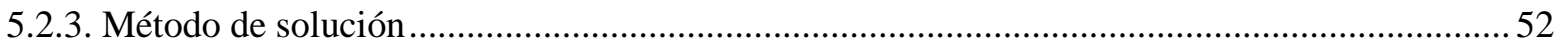

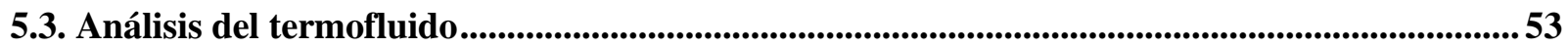

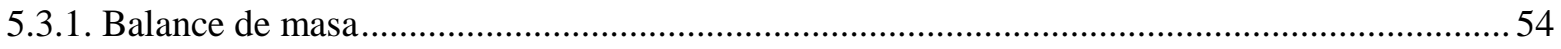

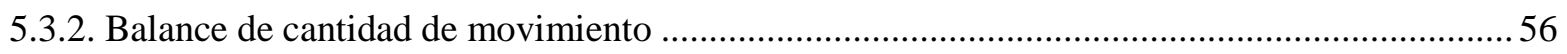

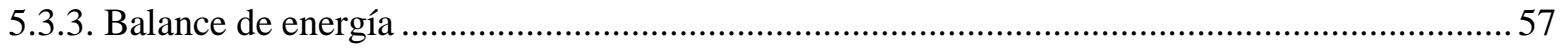

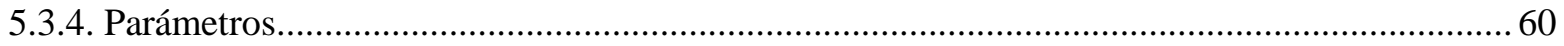

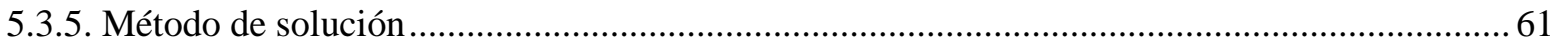

6. Acoplamiento e Implementación .........................................................................................................63

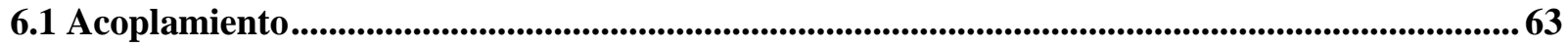

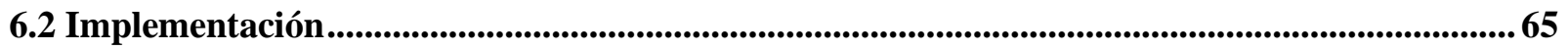

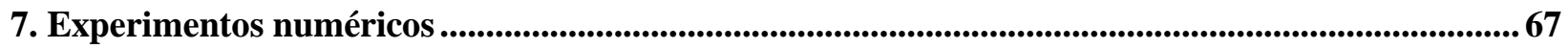

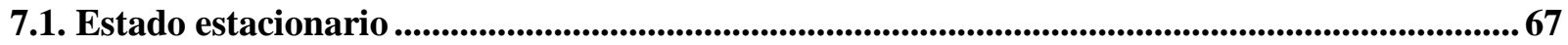

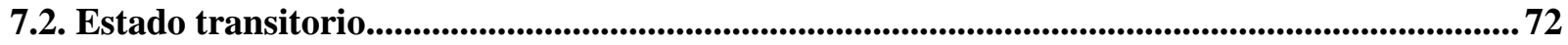

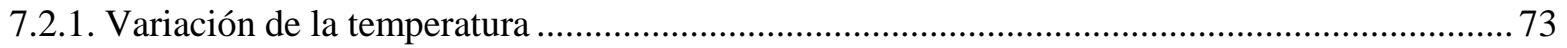

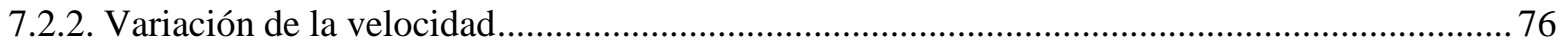

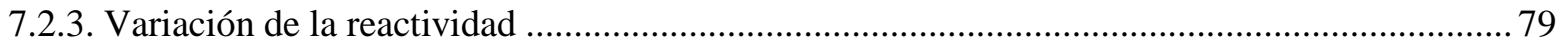

8. Análisis de sensibilidad e incertidumbre .............................................................................................83

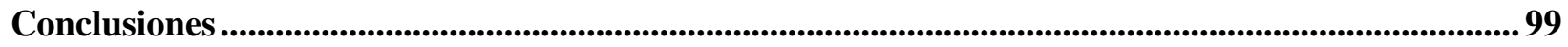

Recomendaciones o Perspectivas ................................................................................................................. 103

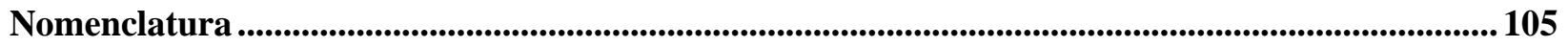

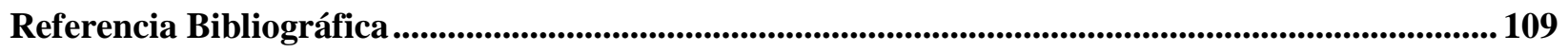




\section{Índice de figuras}

Figura 1.1. Porcentaje de generación de energía eléctrica por fuente de energía............................................5

Figura 1.2. Histórico de la generación de electricidad por fuentes nucleares.................................................

Figura 1.3. Distribución actual de los tipos de reactores. (BWR: reactor de agua en ebullición; FBR: reactor rápido; GCR: reactor refrigerado por gas; LWGR: reactor refrigerado por agua ligera y moderado por grafito; PHWR: reactor de agua pesada a presión; PWR: reactor de

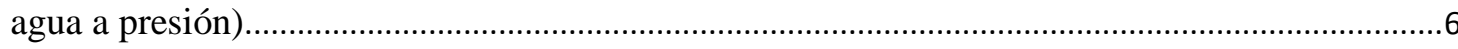

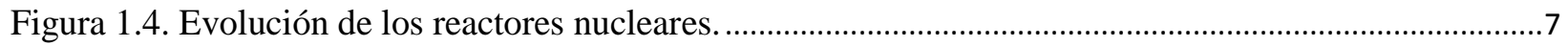

Figura 1.5. Mapa conceptual de los reactores de Generación IV. .....................................................................9

Figura 1.6. Esquema general del Reactor de Temperatura Muy Alta ............................................................12

Figura 1.7. Esquema general del Reactor Rápido enfriado con Sodio .........................................................13

Figura 1.8. Esquema general del Reactor Rápido enfriado con Agua Supercrítica .......................................14

Figura 1.9. Esquema general del Reactor Rápido enfriado con plomo ........................................................15

Figura 1.10. Esquema general del Reactor de Sal fundida ..........................................................................16

Figura 1.11. Esquema general del Reactor Rápido enfriado por Gas ...........................................................17

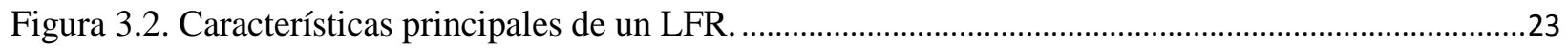

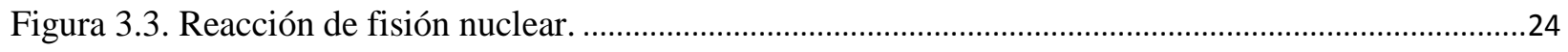

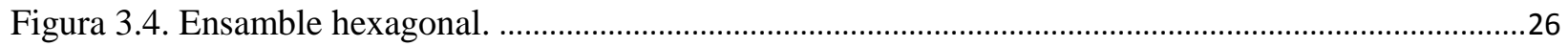

Figura 3.5. Barra de combustible del reactor ALFRED (izquierda), sección transversal de una barra de

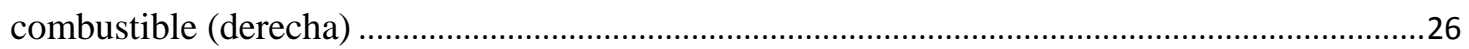

Figura 3.6. Configuración del núcleo del reactor ALFRED .........................................................................27

Figura 4.1. Perfil de la densidad del plomo a diferentes temperaturas ..........................................................34

Figura 4.2. Perfil del coeficiente de expansión del plomo a diferentes temperaturas .....................................35

Figura 4.3. Perfil de la capacidad calorífica del plomo a diferentes temperaturas..........................................36

Figura 4.4. Perfil de la entalpía del plomo a diferentes temperaturas ...........................................................37

Figura 4.5. Perfil de la viscosidad del plomo a diferentes temperaturas........................................................38

Figura 4.6. Perfil de la conductividad térmica del plomo a diferentes temperaturas.....................................38

Figura 5.1.Distribución de potencia axial en la barra de combustible. ............................................................40

Figura. 5.2. Radios de cada sección en la barra de combustible ....................................................................49

Figura 5.3. Sistema para el análisis termofluido. ...................................................................................

Figura 5.4. Barra de combustible en arreglo hexagonal............................................................................6

Figura 5.5. Canal promedio para el análisis termofluido...........................................................................61

Figura. 6.1. Acoplamiento Multi-físico ................................................................................................... 
Figura 6.2. Diagrama de variables para el acoplamiento multi-físico.

Figura 6.3. Diagrama del código PLOMO_REACTOR por llamadas............................................................66

Figura 7.1. Temperatura del combustible (fuel), gap y encamisado (clad) en estado estacionario. .............68

Figura 7.2. Perfil axial de temperatura del combustible (fuel), gap encamisado (clad) y refrigerante (lead) en estado estacionario a100\% de potencia.

Figura 7.3. Distribución de temperatura en la coordenada radial y axial de la barra de combustible a $100 \%$ de potencia..

Figura 7.4. Distribución radial de temperatura en la barra de combustible a diferentes potencias de operación a $0.36 \mathrm{~m}$.

Figura 7.5. Perfil axial de temperatura a $100 \%, 75 \%, 50 \%$ y $25 \%$ de potencia de la sección a) combustible, b) gap, c) clad y d) refrigerante.

Figura 7.6. Campo de temperaturas de temperatura en una barra de combustible a $25 \%, 50 \%, 75 \%$ y $100 \%$ de potencia.

Figura 7.7. Potencia normalizada durante una perturbación en la temperatura del refrigerante....................73

Figura 7.8. Reactividades durante la perturbación de la temperatura. .............................................................74

Figura 7.9. Acercamiento del comportamiento de reactividades en una perturbación de temperatura........75

Figura 7.10. Reactividad total durante una perturbación en la temperatura del refrigerante........................76

Figura 7.11. Generación de percusores durante una perturbación en la temperatura del refrigerante. .........76

Figura 7.12. Potencia (normalizada) durante una perturbación en la velocidad del refrigerante..................77

Figura 7.13. Reactividad total durante una perturbación velocidad del refrigerante. ....................................77

Figura 7.14. Reactividades en una perturbación de velocidad de refrigerante. …............................................78

Figura 7.15. Acercamiento del comportamiento de reactividades en una perturbación de velocidad del refrigerante.

Figura 7.16.Concentración de precursores durante una perturbación en la velocidad del refrigerante.........79

Figura 7.17. Potencia (normalizada) durante la perturbación de reactividad..................................................80

Figura 7.18. Reactividad total durante la perturbación de la reactividad. .....................................................80

Figura 7.19. Acercamiento del comportamiento de reactividades en una perturbación de reactividad. .......81

Figura 7.20. Reactividades en una perturbación de reactividad..................................................................82

Figura 7.21. Concentración de precursores durante la perturbación de la reactividad. ..................................82

Figura 8.1.Variación de la reactividad para determinar el tiempo de estabilidad. ........................................83

Figura 8.2. Números pseudoaleatorios distribuidos uniformemente en el espacio $\mathrm{U}(0,1)$..........................86

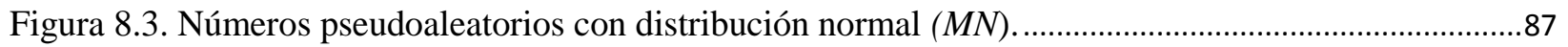

Figura 8.4. Frecuencia de los números pseudoaleatorios en una distribución normal....................................87

Figura 8.5. Distribución de los valores de flujo generados...........................................................................8 
Figura 8.6. Distribución de los valores de temperatura generados.

Figura 8.7. DER del flujo de entrada $\left(W_{i n}\right)$ y de la temperatura de entrada del refrigerante $\left(T_{\text {lead }}\right)$........91

Figura 8.8. Distribución de los valores de a) potencia, b) reactividad, c) temperatura de entrada del refrigerante y d) temperatura máxima de clad generados con la variación del flujo....................93

Figura 8.9. Distribución de los valores de a) potencia, b) reactividad, c) temperatura de entrada del refrigerante y d) temperatura máxima de clad generados con la variación de temperatura de entrada del refrigerante.

Figura 8.10. Desviación estándar relativa (DER) para $P, T_{\text {lead }_{\text {out }}}, T_{\text {clad }_{\max }}$ con variación de flujo de entrada.

Figura 8.11. Desviación estándar relativa (DER) para $P, T_{\text {lead }_{\text {out }}}, T_{\text {clad }_{\max }}$ con variación de temperatura de entrada. . .95

Figura 8.12. Efecto de la variación del flujo de refrigerante sobre: a) la potencia, b) la reactividad, c) temperatura de salida del refrigerante y d) temperatura máxima de clad. . .96

Figura 8.13. Efecto de la variación de la temperatura de entrada del refrigerante sobre: a) la potencia, b) la reactividad, c) temperatura de salida del refrigerante y d) temperatura máxima de clad. .97 


\section{Índice de tablas}

Tabla 1.1. Principales características de los sistemas de Generación IV .......................................................10

Tabla 1.2. Objetivos de los reactores de Generación IV .............................................................................11

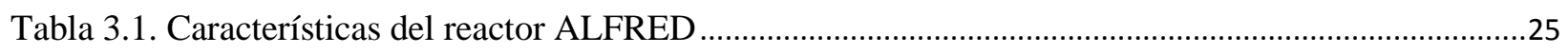

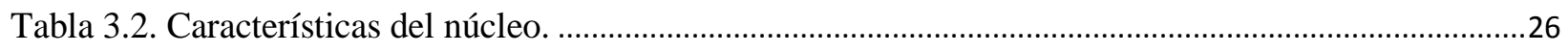

Tabla 4.1. Constantes para el cálculo de la densidad......................................................................................30

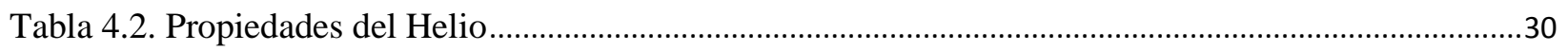

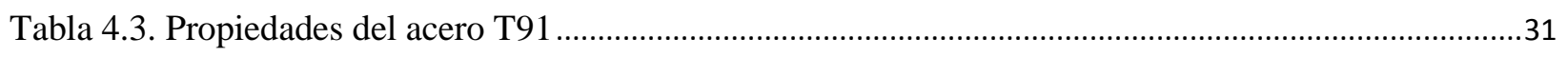

Tabla 5.1. Parámetros de los neutrones retardados para el Plutonio-239 ….................................................47

Tabla 5.2. Parámetros termofluido por canal promedio............................................................................61

Tabla 5.3. Números adimensionales para la transferencia de calor al refrigerante.......................................61

Tabla 7.1. Diferencia de temperatura radial inicial y final de las secciones de la barra...............................70

Tabla 8.1. Valor máximo, mínimo e incertidumbre generados para la variación de flujo del refrigerante. 92

Tabla 8.2. Valor máximo, mínimo e incertidumbre generados para la variación del temperatura de

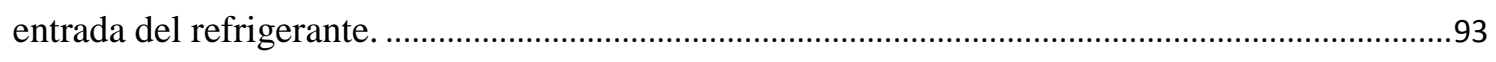

Tabla 8.3. Correlaciones entre las variables de entrada y las variables de salida, y valor de $R^{2} \ldots \ldots \ldots \ldots \ldots \ldots . . .98$ 


\section{Resumen}

En la actualidad, la demanda energética crece a medida que la población aumenta, se estima que dentro de algunas décadas, los recursos fósiles no serán suficientes para satisfacer esta demanda. Esto ha motivado a muchos países en realizar investigación para el diseño y construcción para la aplicación de alternativas energéticas, tales como solar, eólica, biomasa y nuclear, por citar algunas.

Dentro de las tecnologías para generación de energía con base en fuentes nucleares, se han propuesto los reactores de Generación IV (GIV), estos reactores pueden ayudar a satisfacer la demanda de energía preservando en gran medida el ambiente. Por tal motivo es importante el estudio y desarrollo de estos reactores.

El objetivo de este trabajo fue el desarrollo de un modelo matemático que describe los fenómenos de un reactor nuclear enfriado con plomo, el cual es clasificado como un reactor de GIV. En el análisis se consideraron los procesos neutrónicos, los procesos de transferencia de calor en la barra de combustible y los procesos de transferencia de calor en el refrigerante (termofluido). Este análisis permitió desarrollar un modelo multi-físico y multi-escala.

Para los procesos neutrónicos se utilizó el modelo puntual de la cinética neutrónica con seis grupos de precursores de neutrones retardados, que considera efectos de reactividad por efecto Doppler y expansión de combustible nuclear, incluyendo el huelgo u holgura ("gap") y encamisado ("clad"). El modelo de transferencia de calor en la barra de combustible considera la conducción de calor en el combustible y en el clad, y los efectos de convección del gas en el gap y del plomo líquido como refrigerante. El termofluido (refrigerante) fue modelado a partir de los balances de masa con efectos de acumulación y convectivos, cantidad de movimiento y energía en régimen transitorio y unidimensional en la dirección promedio del fluido.

Posteriormente se llevó a cabo la etapa de acoplamiento, en donde se relacionan cada uno de los procesos a partir de las variables que comparten. Con el modelo acoplado se realizaron experimentos numéricos en estado estacionario a diferentes niveles de potencia, también se ejecutaron experimentos para analizar el comportamiento transitorio en condiciones de pérdida de refrigerante, pérdida de recalentadores y la inserción o extracción de barras de control.

Finalmente se realizó un análisis de sensibilidad e incertidumbre con técnicas de simulación Monte Carlo. La sensibilidad e incertidumbre se analizó con flujo y temperatura de entrada al núcleo del reactor. 


\begin{abstract}
At present, energy demand grows as the population increases, it is estimated that in some decades fossil resources will not be enough to satisfy the demand. For this reason, many countries have been motivated to carry out research in design and develop for the application of energy alternatives like solar, wind, biomass and nuclear.
\end{abstract}

Within nuclear technologies, the Generation IV reactors (GIV) have been proposed. These reactors can help to satisfy energy demand while care the environment. For this reason, study and development of these reactors are important.

The objective of this work was the development of a mathematical model that describes the phenomena of a lead-cooled fast reactor (LFR), which is classify as a GIV reactor. The analysis of neutron processes, heat transfer processes in the fuel rod and heat transfer processes in the coolant (thermofluid), were considered. This analysis allowed the development of a multi-physics and multi-scale model.

For the neutron processes, was used neutron point kinetics model with six precursors of delayed neutrons, which considers effects of Doppler reactivity and expansion of nuclear fuel, including gap and clad expansion. The heat transfer model in fuel rod considers conduction of heat in fuel and clad, and considers also convection effects of gas gap and liquid lead. The thermofluid (coolant) in core was modeled from mass balance whit accumulation and convective effects, momentum balance and energy balance in transient and one-dimensional regime in direction of the mean fluid.

Subsequently, the coupling step was carried out; where the processes are related from the variables they share (interactions between models). With the coupled model, numerical experiments were performed at steady state whit different power levels. Experiments were also carried out to analyze the transient behavior under conditions of loss of coolant, loss of reheaters and control bar drop (Insertion of positive reactivity).

Finally, a sensitivity and uncertainty analysis was performed using Monte Carlo simulation techniques. Sensitivity and uncertainty were analyzed with inflow and inlet temperature to the core reactor. 


\section{Introducción}

Debido a las crecientes preocupaciones sobre la disponibilidad de recursos energéticos, el cambio climático y la seguridad energética, y puesto que la energía nuclear puede hacer una aportación importante en la tarea de satisfacer la creciente demanda mundial de energía, en las últimas décadas se han puesto en marcha diversas iniciativas internacionales en relación con los sistemas de energía nuclear innovadores.

En particular, con el nuevo siglo, varios Estados Miembros del Organismo Internacional de Energía Atómica (OIEA) reconocieron la necesidad de actuar para asegurar que el desarrollo de la energía nuclear se hiciera de forma sostenible. Como resultado de ello, en el año 2000 se inició el Proyecto Internacional sobre ciclos del combustible y reactores nucleares innovadores (INPRO), a fin de ayudar a asegurar la disponibilidad de energía nuclear para satisfacer las necesidades mundiales de energía en el siglo XXI de manera sostenible (IAEA, 2016). El objetivo principal era reunir a los propietarios y a los usuarios de tecnologías a fin de que analizaran juntos las medidas internacionales y nacionales necesarias para lograr las innovaciones deseadas en los reactores nucleares y los ciclos del combustible.

El Foro Internacional de la Generación IV (GIF) es el resultado de un esfuerzo internacional de cooperación realizado con el fin de llevar a cabo las actividades de investigación y desarrollo necesarias para determinar la viabilidad y la capacidad de rendimiento de la próxima generación de sistemas de energía nuclear. Con sus 13 miembros, el GIF se centra en seis sistemas de energía nuclear, tal como se describe en su documento titulado Technology Roadmap for Generation IV Nuclear Energy Systems, publicado en el año 2002: los reactores rápidos refrigerados con gas (GFR), los reactores de muy alta temperatura (VHTR), los reactores refrigerados con agua supercrítica (SCWR), los reactores rápidos refrigerados con sodio (SFR), los reactores rápidos refrigerados con plomo (LFR) y los reactores de sales fundidas (MSR) (GIF 2002).

En este proyecto se realiza una contribución al modelo del reactor rápido enfriado con plomo (LFR) realizando un análisis para modelar matemática y numéricamente los procesos que ocurren en el núcleo del reactor. El análisis está dividido en cuatro partes principales, la primera es un análisis de los procesos neutrónicos a partir de un modelo de orden reducido de la cinética neutrónica, la segunda y tercera etapa son un análisis de los procesos de transferencia de calor en el combustible y análisis de los procesos del termofluido en donde se estudia el comportamiento del plomo como refrigerante. Posteriormente se realiza la etapa de acoplamiento, en donde se analiza la interacción de los análisis previos con la finalidad de obtener una retroalimentación de cada uno. 
Con el modelo acoplado se llevan a cabo experimentos en estado estacionario para determinar las temperaturas del combustible, encamisado y refrigerante, y compararlas con los límites de diseño.

También se realizan experimentos en estado transitorio para simular eventos de pérdida de refrigerante, accidente por falla de los calentadores del refrigerante y la inserción y extracción de barras de control, mostrando el comportamiento seguro de la potencia del reactor, es decir, que después del estado transitorio la potencia se estabilice nuevamente.

Además de experimentación numérica, se realizó un análisis de sensibilidad e incertidumbre aplicando métodos de simulación Monte Carlo, con la finalidad de obtener la incertidumbre y correlaciones de las variables de salida (potencia, reactividad y temperaturas del clad y refrigerante) en función de las variables de entrada principales para análisis de seguridad (temperatura y flujo de entrada del refrigerante).

En varios países se realizan desarrollos de los LFR, para esta investigación se toma como referencia el reactor ALFRED (Advanced Lead-cooled Fast Reactor European Demonstrator), éste está en desarrollo en la Unión Europea y es la versión demostrativa del proyecto LEADER (Lead-cooled European Advanced Demonstrator Reactor). 


\section{Antecedentes}

\subsection{Situación energética mundial}

La generación de electricidad se satisface principalmente por combustibles fósiles, lo que ha ocasionado una gran dependencia de éstos, para 2015 la demanda total de electricidad fue de 9,858.4 TWh generados por diferentes fuentes de energía; el $60.6 \%$ generado a partir de combustibles fósiles, el $19.0 \%$ fue nuclear, el $13.8 \%$ a partir de la hidroeléctrica y el $6.6 \%$ restante por fuentes renovables (geotérmica, eólica, solar y biomasa) como se muestra en la Figura 1.1 (IEA, 2015).

\section{Generación de energía a nivel mundial \\ 9,858.4 TWh}

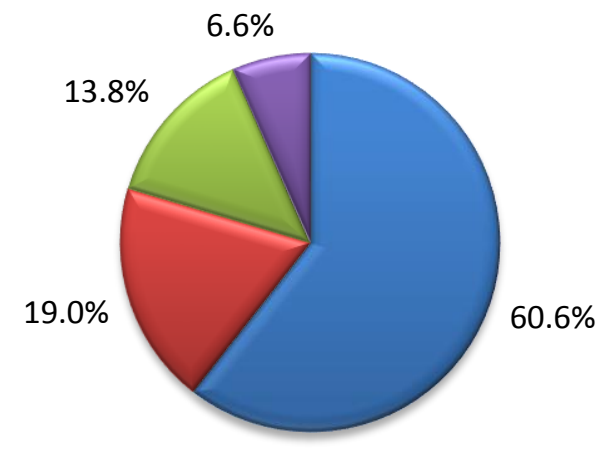

$\square$ Combustibles fósiles

$\square$ Nuclear

$\square$ Hidroeléctrica

$\square$ Geo/solar/eólica/bio

Figura 1.1. Porcentaje de generación de energía eléctrica por fuente de energía. (IEA, 2015).

En la Figura 1.2 se observa un histórico de la generación de energía eléctrica a nivel mundial por medios nucleares. Desde 1985 la generación fue en crecimiento debido a la demanda de energía, lo que motivó a la apertura de nuevas centrales, pero después del 2010 la generación disminuyó ya que se cerraron varias centrales. Sin embargo, actualmente se encuentran en construcción 60 reactores nucleares (IAEA, 2017).

Por otra parte, la energía nuclear ha contribuido constantemente con la generación de electricidad. Actualmente hay en funcionamiento 449 reactores nucleares de potencia en todo el mundo, con una capacidad total instalada de 392, 209 MWe (IAEA, 2017). De los reactores comerciales en funcionamiento, alrededor del $81.6 \%$ son reactores moderados y refrigerados por agua ligera, un $11.1 \%$ son reactores moderados y refrigerados por agua pesada, un $3.4 \%$ son reactores refrigerados por agua ligera y moderados por grafito, y un $3.1 \%$ son reactores refrigerados por gas y $0.7 \%$ son reactores rápidos refrigerados por metal líquido (Figura 1.3). 


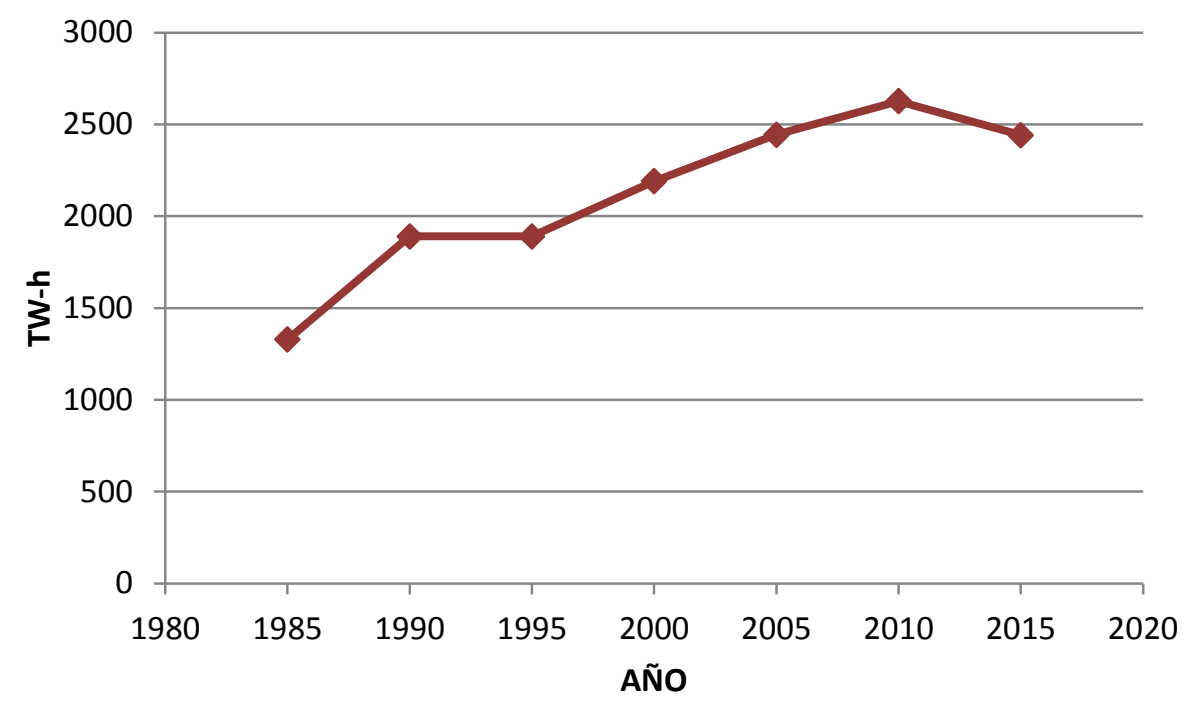

Figura 1.2. Histórico de la generación de electricidad por fuentes nucleares (IAEA, 2016).

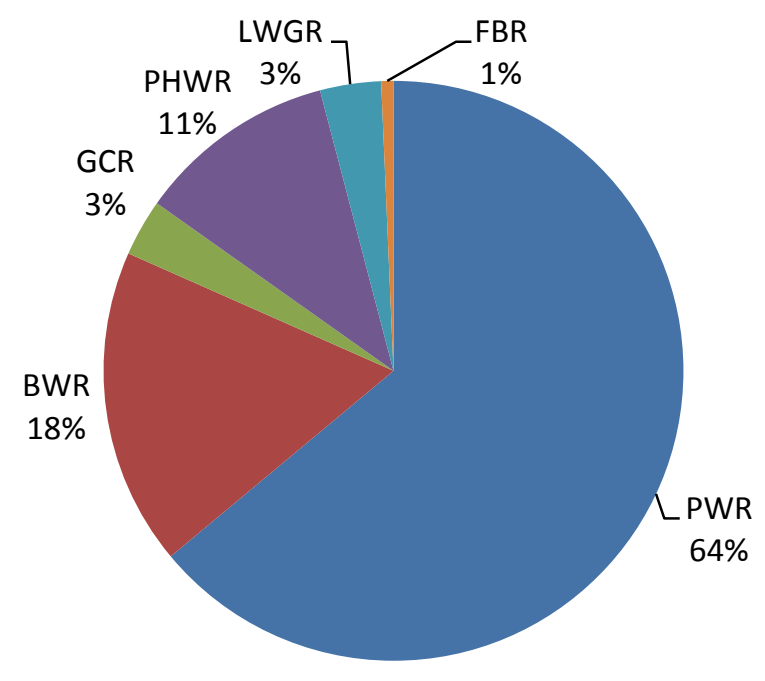

Figura 1.3. Distribución actual de los tipos de reactores. (BWR: reactor de agua en ebullición; FBR: reactor rápido; GCR: reactor refrigerado por gas; LWGR: reactor refrigerado por agua ligera y moderado por grafito; PHWR: reactor de agua pesada a presión; PWR: reactor de agua a presión) (IAEA, 2016). 


\subsection{Evolución de los Reactores Nucleares}

El desarrollo de la tecnología nuclear se inició alrededor de 1950, en donde se crearon los primeros reactores nucleares de experimentación, a estos reactores se les conoce como de Generación I (Figura 1.4) (GIF, 2009).

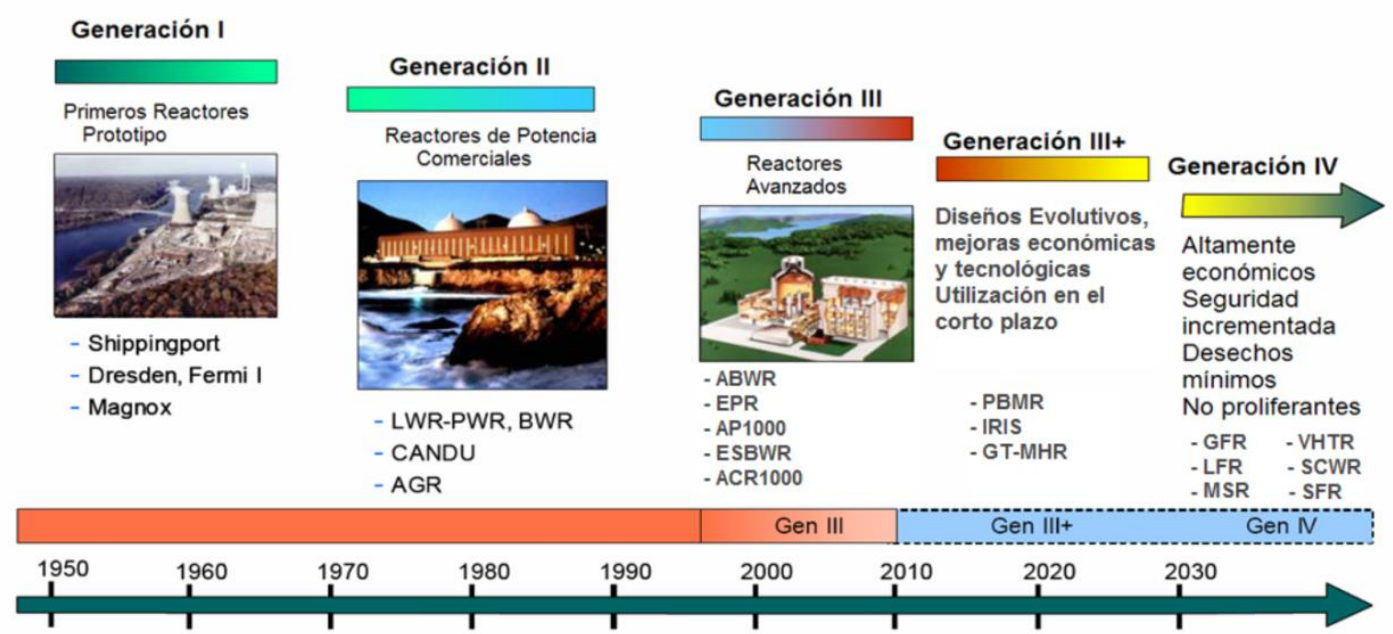

Figura 1.4. Evolución de los reactores nucleares (GIF, 2009).

A partir de la década de 1970 se crearon los reactores de generación II, éstos se caracterizan por ser los primeros reactores comerciales para la generación de electricidad, los sistemas desarrollados fueron el BWR (Boiling Water Reactor), PWR (Pressurized Water Reactor), los cuales son LWR (Light Water Reactor). En esta generación se creó el reactor CANDU (CANada Deuterium Uranium).

La Generación III se considera a partir del año 2000, en esta generación se mejoraron los diseños de la Generación II. A los reactores desarrollados en esta generación se les conoce como reactores avanzados. Seguido a estos reactores se tiene a la Generación III+, en la cual el objetivo es crear reactores con diseños evolutivos, en donde se obtengan mejoras económicas de forma que sean más competitivos y que las mejoras tecnológicas permitan construirlos en menos tiempo. En las generaciones III y III+, en donde se crean los reactores avanzados de agua y reactores enfriados por gas, el diseño de los reactores mantiene las características básicas de operación pero mejorando la fiabilidad en su operación y sistemas de seguridad.

Estos reactores se dividen en dos clases: 1) diseños usando sistemas de seguridad activados con energía y 2) diseños que utilizan sistemas de seguridad pasivos, característica que se basan en fuerzas naturales, por ejemplo, flujos movidos por gravedad o conducción térmica (Todreas y Kazimi, 2012). 
Los reactores térmicos avanzados y reactores de espectro rápido de neutrones se encuentran dentro de la Generación IV. Éstos fueron seleccionados por la el diseño del reactor, la conversión de energía y el tipo de combustible, pero esto no significa que sean de reciente diseño, estos reactores comenzaron su diseño a mediados del siglo XX y a inicios del siglo XXI se consideraron para formar parte de la siguiente generación de reactores. Los diseños incluyen núcleos con neutrones térmicos y neutrones rápidos así como ciclos de combustibles abiertos y cerrados y diversos refrigerantes. Se espera que los primeros reactores GIV comerciales se tengan alrededor de 2030-2040 (GIF, 2016).

A pesar de que estos reactores son la última generación en reactores de fisión, algunos de ellos ya han sido construidos y probados anteriormente. De los seis sistemas, todos tienen proyectos existentes, es decir, se está trabajando en su diseño para construirlos en un futuro.

Los reactores rápidos tienen sus primeros orígenes a mediados de la década de 1950, en donde se utilizaron los reactores enfriados con plomo como fuente de energía para submarinos en Rusia. El primer reactor rápido conocido, que fue también el primero en utilizar plutonio-239 como combustible, se construyó en Los Álamos para trabajos experimentales en los que se necesitaba una buena fuente de neutrones rápidos. A este reactor se le dio el nombre de "Clementine". Comenzó a funcionar en noviembre de 1946, con una potencia de 10kW, incrementada posteriormente, en marzo de 1949, a 25kW. El núcleo del reactor, cilíndrico, tenía aproximadamente 6 pulgadas de diámetro por 6 pulgadas de altura, y constaba de cierto número de barras verticales de plutonio con vainas de acero. Como refrigerante se empleó mercurio. El reactor fue desmantelado en 1953, como consecuencia de la rotura de uno de los elementos combustibles y de la contaminación del mercurio resultante (Glassone y Sesonske, 1990).

Posteriormente comenzó el desarrollo para utilizarlos como reactores de cría, es decir, reactores generadores de combustible, seguido de su utilización como reactores de quema de combustible y generación de energía eléctrica. El primer prototipo de reactor rápido de potencia fue el EBR-I (Experimental Breeder Reactor-I), que alcanzó la criticidad en agosto de 1951. Su objetivo era el de proporcionar información respecto a las posibilidades de reproducción con neutrones rápidos y producción simultanea de electricidad, utilizando un metal líquido (aleaciones de Sodio-Potasio, NaK) como refrigerante. La primera energía eléctrica -conocida- de origen nuclear la produjo el EBR-I, en diciembre de 1951. El núcleo de éste reactor estaba constituido por cierto número de barras de uranio altamente enriquecido, con todo el conjunto rodeado por una capa de uranio natural. Su potencia térmica era de 1.4 MW. Aunque se trataba de un reactor pseudo-reproductor, consiguió plenamente su objetivo, demostrando que era factible la reproducción y la producción simultanea de energía, utilizando refrigeración por medio de metales líquidos. 
Desde 1960 se han implementado en todo el mundo importantes programas de desarrollo y utilización de los reactores rápidos, que han hecho que los conocimientos sobre los reactores rápidos y las tecnologías del ciclo del combustible conexas alcancen un alto grado de madurez. La tecnología de reactores rápidos más madura es el reactor rápido refrigerado por sodio (SFR). Su historia es la de 350 años-reactor de experiencia adquirida a través del diseño, la construcción y la explotación de SFR experimentales, prototípicos, de demostración y de tamaño comercial que hay en funcionamiento en varios países, como Alemania, China, los Estados Unidos de América, la Federación de Rusia, Francia, la India, el Japón y el Reino Unido.

En líneas generales, los resultados de los SFR han sido notables, con logros importantes, como la demostración de la viabilidad de la reproducción de combustible nuevo a través del ciclo del combustible de los reactores rápidos, con eficiencias térmicas que alcanzan valores del 43-45\%, los más elevados en la esfera nuclear. Asimismo, la clausura de varios de estos reactores ha permitido acumular experiencia indispensable.

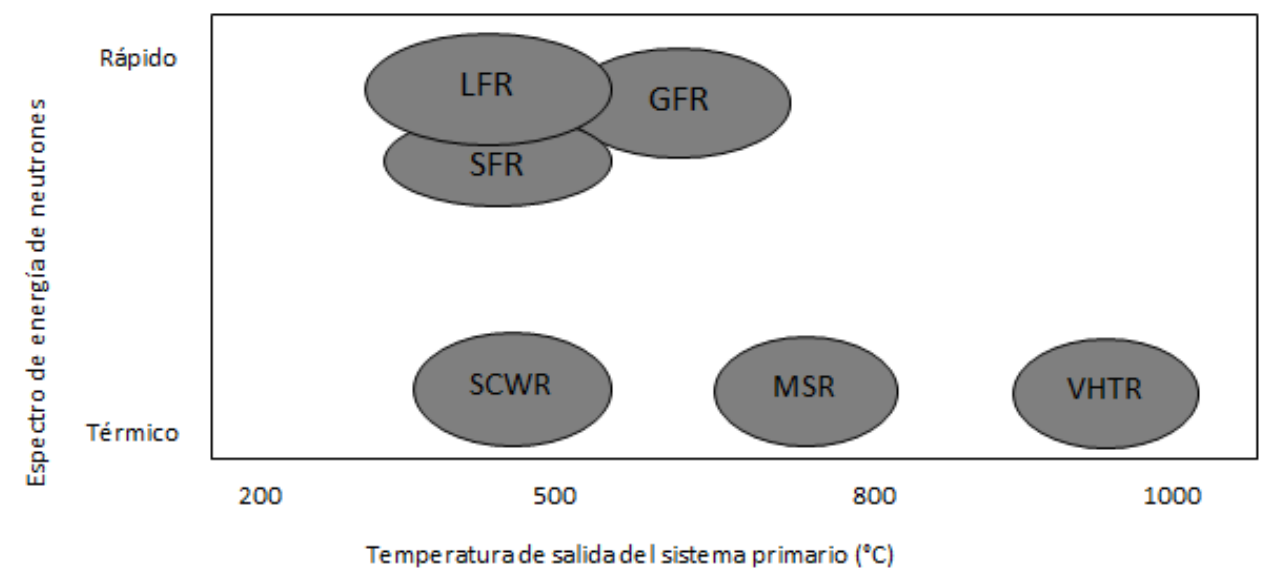

Figura 1.5. Mapa conceptual de los reactores de Generación IV (Adaptado de Todreas y Kazimi, 2012).

Las funciones de estos reactores incluyen producción de electricidad, calor de proceso para generación de hidrógeno así como un manejo de desechos por trasmutación de actínidos (neptunio, plutonio, americio y curio). Las principales características del reactor son la energía media del rector y la temperatura de salida del sistema primario (Figura 1.5).

\subsection{Reactores Nucleares de Generación IV}

Como se comentó anteriormente, dentro de los reactores GIV existen 6 sistemas mostrados en la Tabla 1.1, existe un sistema que trabaja con un espectro de neutrones térmico, dos sistemas con espectro térmico 
o rápido y tres sistemas con neutrones rápidos. Algunos de estos sistemas están diseñados para poder trabajar en rangos bajos y altos de potencia.

Tabla 1.1. Principales características de los sistemas de Generación IV (GIF, 2016).

\begin{tabular}{cccccc}
\hline Sistema & $\begin{array}{c}\text { Espectro de } \\
\text { Neutrones }\end{array}$ & Refrigerante & $\begin{array}{c}\text { Temperatura } \\
\left({ }^{\circ} \mathbf{C}\right)\end{array}$ & Combustible & $\begin{array}{c}\text { Tamaño } \\
(\mathbf{M W e})\end{array}$ \\
\hline $\begin{array}{c}\text { VHTR (Reactor de } \\
\text { muy alta temperatura) }\end{array}$ & Térmico & Helio & $900-1000$ & UO2 & $250-300$ \\
\hline $\begin{array}{c}\text { SFR (Reactor rápido } \\
\text { enfriado por sodio) }\end{array}$ & Rápido & Sodio & 550 & MOX & $30-150$ \\
\hline $\begin{array}{c}\text { SCWR (Reactor } \\
\text { enfriado con agua } \\
\text { supercrítica) }\end{array}$ & Térmico/Rápido & Agua & $510-625$ & UO2 & $1000-2000$ \\
\hline $\begin{array}{c}\text { GFR (Reactor rápido } \\
\text { enfriado por gas) }\end{array}$ & Rápido & Helio & 850 & MOX & $1000-1500$ \\
\hline $\begin{array}{c}\text { LFR (Reactor rápido } \\
\text { enfriado por plomo) }\end{array}$ & Rápido & Plomo & $480-800$ & MOX & 1200 \\
\hline $\begin{array}{c}\text { MSR (Reactor de sal } \\
\text { fundida) }\end{array}$ & Térmico/Rápido & Sales de & fluoruro & & \\
\hline
\end{tabular}

El desarrollo de los reactores de Cuarta Generación (GIV) tiene ocho objetivos principales (Tabla 1.2) distribuidos en cuatro áreas: sustentabilidad, economía, seguridad y fiabilidad además de la resistencia a la proliferación y la protección física (GIF, 2016). Estos objetivos están destinados a dar respuesta a las necesidades económicas, ambientales y sociales del Sigo XXI.

Estos objetivos guían los esfuerzos de investigación y desarrollo llevados a cabo principalmente por los países miembros de GIF ${ }^{1}$ (Generation IV International Forum). Los retos planeados están destinados a estimular dichos esfuerzos considerando todos los aspectos tecnológicos relacionados con el diseño y la implementación de los reactores, los sistemas de conversión de energía y las instalaciones del ciclo de combustible.

\footnotetext{
${ }^{1}$ El Foro internacional de Generación IV surgió a partir de la necesidad de enfocar esfuerzos para la investigación y desarrollo de reactores nucleares de cuarta generación, se fundó en el año 2000 y actualmente cuenta con doce países miembros y la representación de la Comunidad Europea. Para más información véase https://www.gen4.org/gif/jcms/c_9260/Public.
} 
Tabla 1.2. Objetivos de los reactores de Generación IV (GIF, 2016).

\begin{tabular}{|c|c|}
\hline Área & Objetivo específico \\
\hline Sustentabilidad & $\begin{array}{l}\text { Generación de energía de forma sustentable, a modo que cumpla los objetivos } \\
\text { de aire limpio así como ofrecer disponibilidad de los sistemas a largo plazo y } \\
\text { la utilización eficaz del combustible para la producción de energía en todo el } \\
\text { mundo. } \\
\text { Gestión y reducción al mínimo de los residuos nucleares, reducir } \\
\text { notablemente la carga de la administración a largo plazo con objeto de } \\
\text { optimizar la protección de la salud pública y el medio ambiente. }\end{array}$ \\
\hline Economía & $\begin{array}{l}\text { Tener una ventaja de costos del ciclo de vida sobre otras fuentes de energía. } \\
\text { Tener un nivel de riesgo financiero comparable a otros proyectos de energía. }\end{array}$ \\
\hline $\begin{array}{l}\text { Seguridad y } \\
\text { confiabilidad }\end{array}$ & $\begin{array}{l}\text { Las operaciones de los reactores sobresaldrán en condiciones de seguridad y } \\
\text { fiabilidad. } \\
\text { Tener baja probabilidad del grado de daño al núcleo del reactor. } \\
\text { Eliminar la necesidad de una respuesta de emergencia fuera del sitio. }\end{array}$ \\
\hline $\begin{array}{l}\text { Resistencia a la } \\
\text { proliferación y la } \\
\text { protección física }\end{array}$ & $\begin{array}{l}\text { Aumentar la seguridad, evitar la desviación o el robo de material utilizable } \\
\text { para armas y proporcionar una mayor protección física contra los actos de } \\
\text { terrorismo. }\end{array}$ \\
\hline
\end{tabular}

El cumplimiento de estos objetivos ayudará al desarrollo de la industria nuclear para generación de electricidad, permitiendo una mejor utilización de los recursos y una mayor aceptación en su utilización.

De acuerdo con el documento "Examen de la Tecnología Nuclear -2014" elaborado por la Agencia Internacional de Energía Atómica (IAEA), actualmente hay cuatro SFR en funcionamiento: el Reactor Experimental Rápido de China, en China; el reactor reproductor rápido de ensayo, en la India; y los reactores BOR-60, BN-600 y BN-800, en la Federación de Rusia. Hay dos SFR en régimen de parada temporal, Joyo y Monju, en Japón.

En la Federación de Rusia se ha reunido algo de experiencia con metales líquidos pesados, como el plomo o la mezcla eutéctica plomo-bismuto, a partir de la explotación de siete submarinos nucleares Project $705 / 705 \mathrm{~K}$ equipados con un reactor refrigerado por $\mathrm{Pb}-\mathrm{Bi}$ de $155 \mathrm{MW}_{\text {th. }}$.

A la fecha se han construido 21 reactores rápidos, cuyo tiempo de operación suma 433 años. Con el esfuerzo de diversos países se han logrado acumular varias décadas de experiencia en el diseño y fabricación de los reactores de cuarta generación (IAEA, 2016). A nivel nacional o internacional se están desarrollando cuatro tipos diferentes de reactores rápidos para cumplir normas de seguridad, sustentabilidad, economía, protección física y resistencia a la proliferación. Estos reactores son: el reactor rápido refrigerado por sodio (SFR), el reactor rápido refrigerado con plomo (LFR), el reactor rápido refrigerado por gas (GFR) y el reactor rápido de sales fundidas (MSR) (IAEA, 2014). 
A continuación se realiza una breve descripción de los reactores de Generación IV.

\section{Reactor de Temperatura Muy Alta (VHTR)}

El VHTR (Very-High Temperature Reactor) (Figura 1.6) es la siguiente generación en desarrollo de reactores de muy alta temperatura y es el primer diseño dedicado a la cogeneración de electricidad, hidrógeno y procesos de calor para la industria. El hidrógeno puede ser extraído del agua usando termoquímica, electro-química o procesos híbridos. El reactor es enfriado con gas helio y moderado por grafito, con una temperatura de salida alrededor de $900^{\circ} \mathrm{C}$ lo cual es necesario para la una producción eficiente de hidrógeno por procesos termoquímicos.

Este tipo de reactores tienen un potencial para un gran quemado de combustible, seguridad pasiva y bajos costos de operación y mantenimiento además de una construcción modular (GIF, 2009).

Las dos opciones base disponibles para el núcleo del VHTR son: tipo lecho de bolas (pebble bed) y el tipo de bloque prismático. La conversión de electricidad puede ser directa (a través de una turbina de gas helio) o indirecto (turbina de mezcla de gases) del ciclo Brayton.

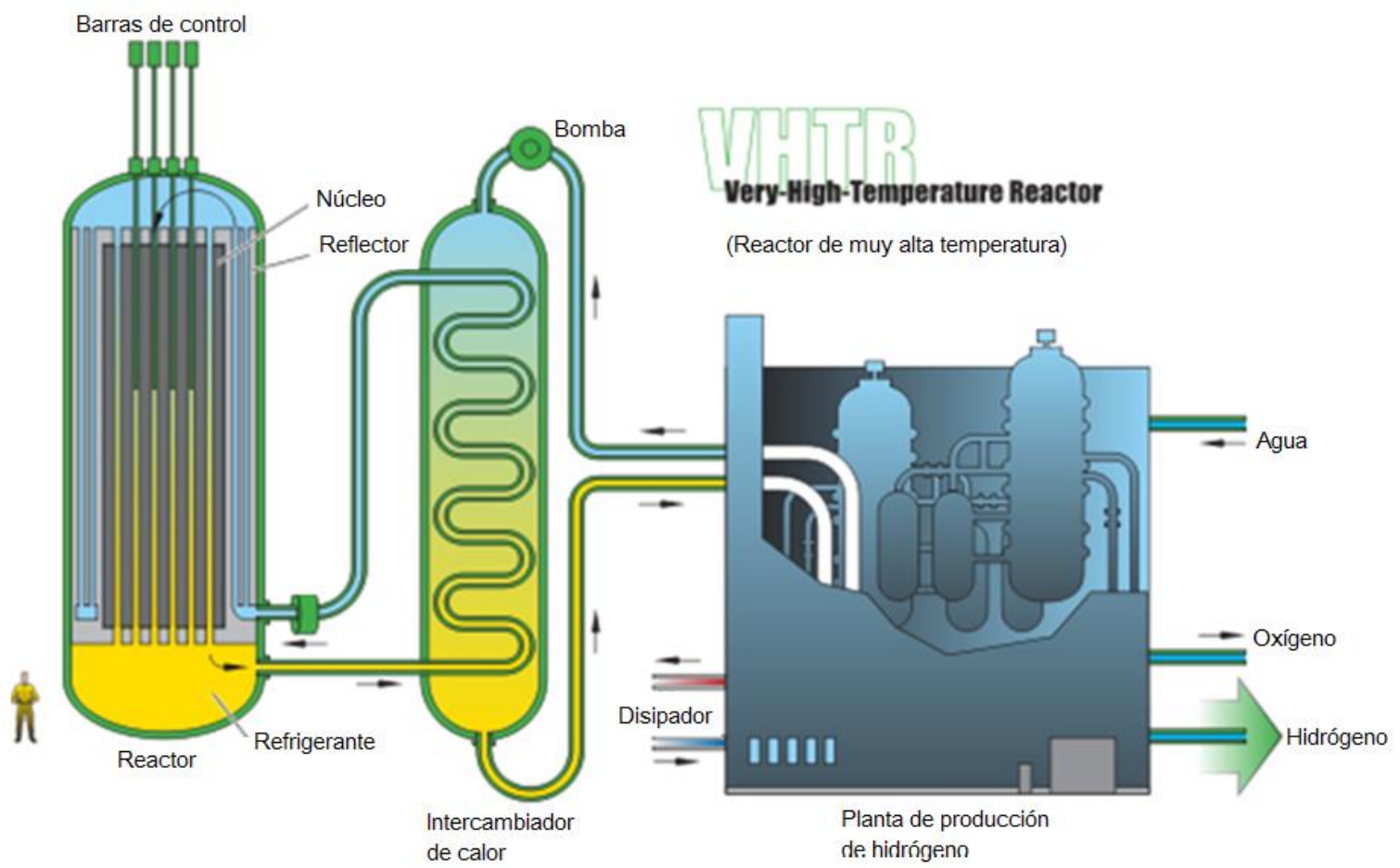

Figura 1.6. Esquema general del Reactor de Temperatura Muy Alta (GIF, 2016). 


\section{Reactor Rápido Enfriado con Sodio (SFR)}

El reactor rápido enfriado por sodio (Sodium-Cooled Fast Reactor) (Figura 1.7) es un proyecto que parte del diseño de dos sistemas de reactores; el reactor reproductor rápido (Liquid Metal Fast Breeder Reactor, LMFBR) y el Reactor rápido integral.

Los reactores rápidos enfriados por sodio usan sodio líquido como refrigerante, permitiendo una alta densidad de poder con una fracción baja de refrigerante además permite al sistema trabajar a presión atmosférica, lo que reduce el riesgo de fugas. Mientras que el ambiente libre de oxigeno previene la corrosión. Es alimentado por un combustible de una aleación metálica de uranio y plutonio (Figura 1.7). El ciclo cerrado de este tipo de reactores permite la regeneración del combustible fisil y facilidades en el manejo de desechos de alto nivel, en particular el plutonio y actínidos menores. Al utilizar un espectro rápido de neutrones extiende en gran medida las fuentes de combustible, comparado con lo reactores térmicos, ya que utiliza una mezcla de óxidos que permite tener Uranio-235, Uranio-238 y Plutonio-239, lo que disminuye la necesidad de enriquecimiento de Uranio y favorece la utilización del Plutonio. El SFR es considerado como el sistema más próximo para un adecuado manejo de actínidos (GIF, 2009).

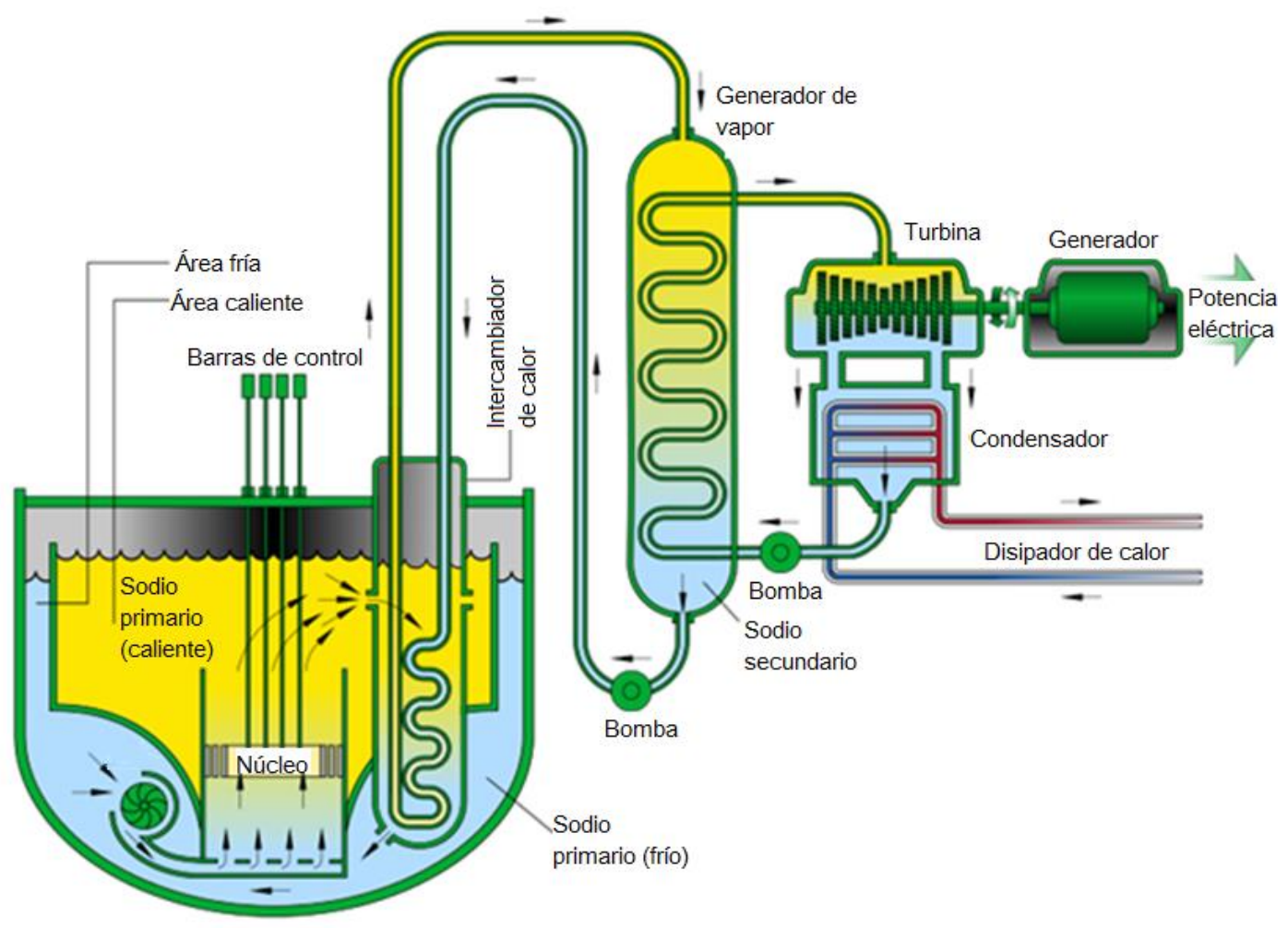

Figura 1.7. Esquema general del Reactor Rápido enfriado con Sodio (GIF, 2016). 


\section{Reactor Enfriado con Agua Supercrítica (SCWR)}

El SCWR (Supercritical-Water-Cooled Reactor) (Figura 1.8) es un reactor de alta temperatura y alta presión enfriado por agua que opera sobre el punto crítico termodinámico del agua $\left(374^{\circ} \mathrm{C}, 22.1 \mathrm{MPa}\right)$. Éste es básicamente un reactor de agua ligera (Light Water Reactor, LWR) operando a mayores presiones y temperaturas.

Como el fluido de operación sólo tiene una fase presente, puede operar a temperaturas mucho más altas de los actuales PWR y BWR. Los reactores enfriados por agua supercrítica son sistemas nucleares avanzados muy prometedores debido a su alta eficiencia termodinámica (arriba del 50\% contra el 34\% de los actuales LWR y considerable simplificación de la planta) (GIF, 2009).

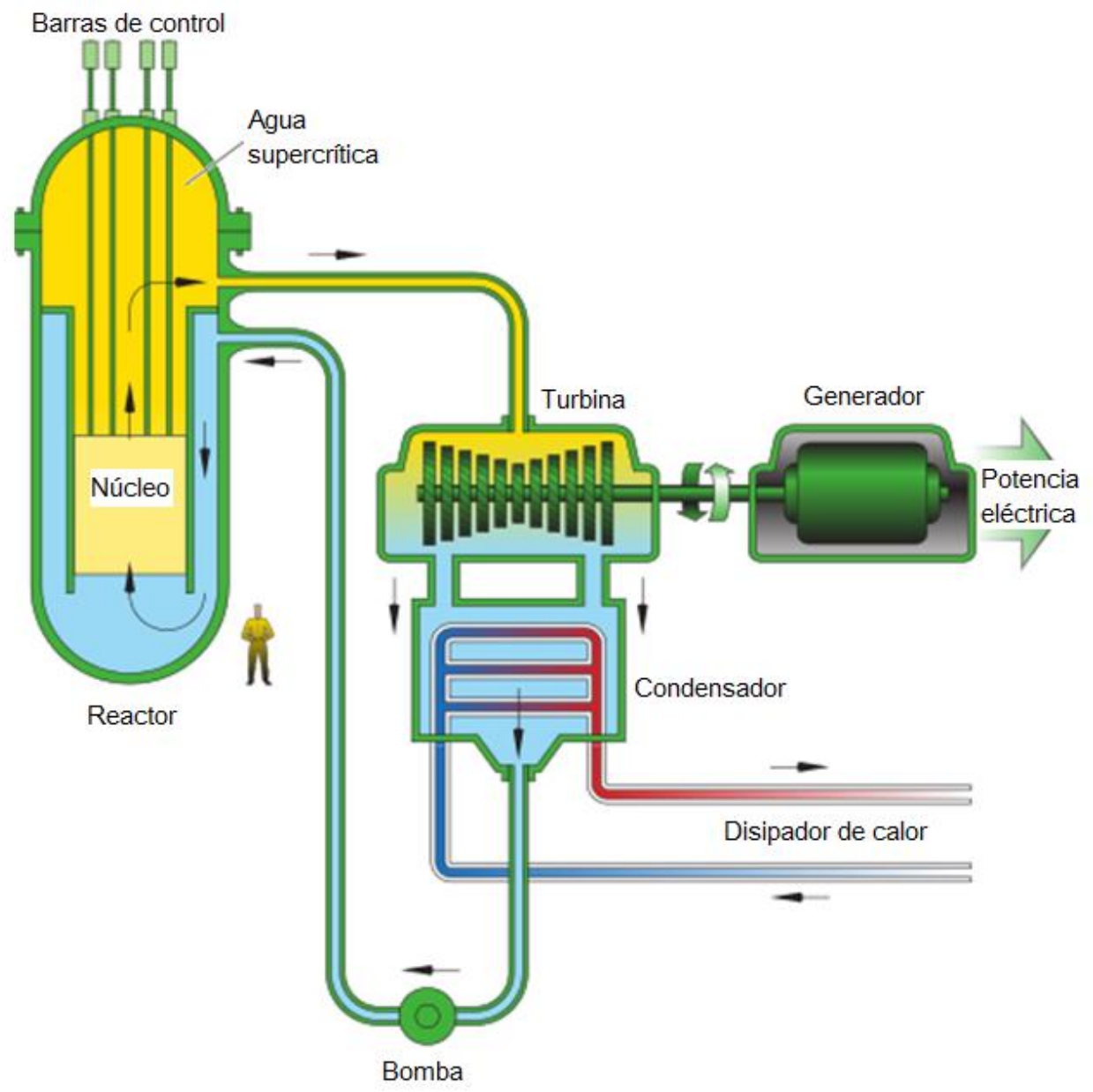

Figura 1.8. Esquema general del Reactor Rápido enfriado con Agua Supercrítica (GIF, 2016). 


\section{Reactor Rápido Enfriado con plomo (LFR)}

Las características del LFR (Lead-Cooled Fast Reactor) (Figura 1.9) son un espectro rápido de neutrones y un ciclo cerrado de combustible para una eficiente conversión del uranio fértil, lo que le da excelentes capacidades de gestión de materiales. Se caracteriza por usar un reactor enfriado por metal líquido de plomo o una mezcla eutéctica de plomo-bismuto. También puede utilizarse como quemador para consumir actínidos a partir de combustible LWR gastado y como quemador/criador con matrices de torio. Una característica importante del LFR es la mayor seguridad que resulta de la elección del plomo fundido como un refrigerante relativamente inerte y de baja presión. En términos de sustentabilidad, el plomo es abundante y, por lo tanto, disponible, incluso en caso de despliegue de un gran número de reactores.

Más importante aún, al igual que con otros sistemas rápidos, la sustentabilidad del combustible se ve reforzada por las capacidades de conversión del ciclo de combustible LFR, debido a que incorporan un refrigerante líquido con un margen muy alto a la ebullición e interacción benigna con el aire o el agua, los conceptos de LFR ofrecen un gran potencial en términos de seguridad, simplificación del diseño, resistencia a la proliferación y los resultados económicos resultantes. Un factor importante es la posibilidad de un estado final benigno a los accidentes graves (GIF, 2009).

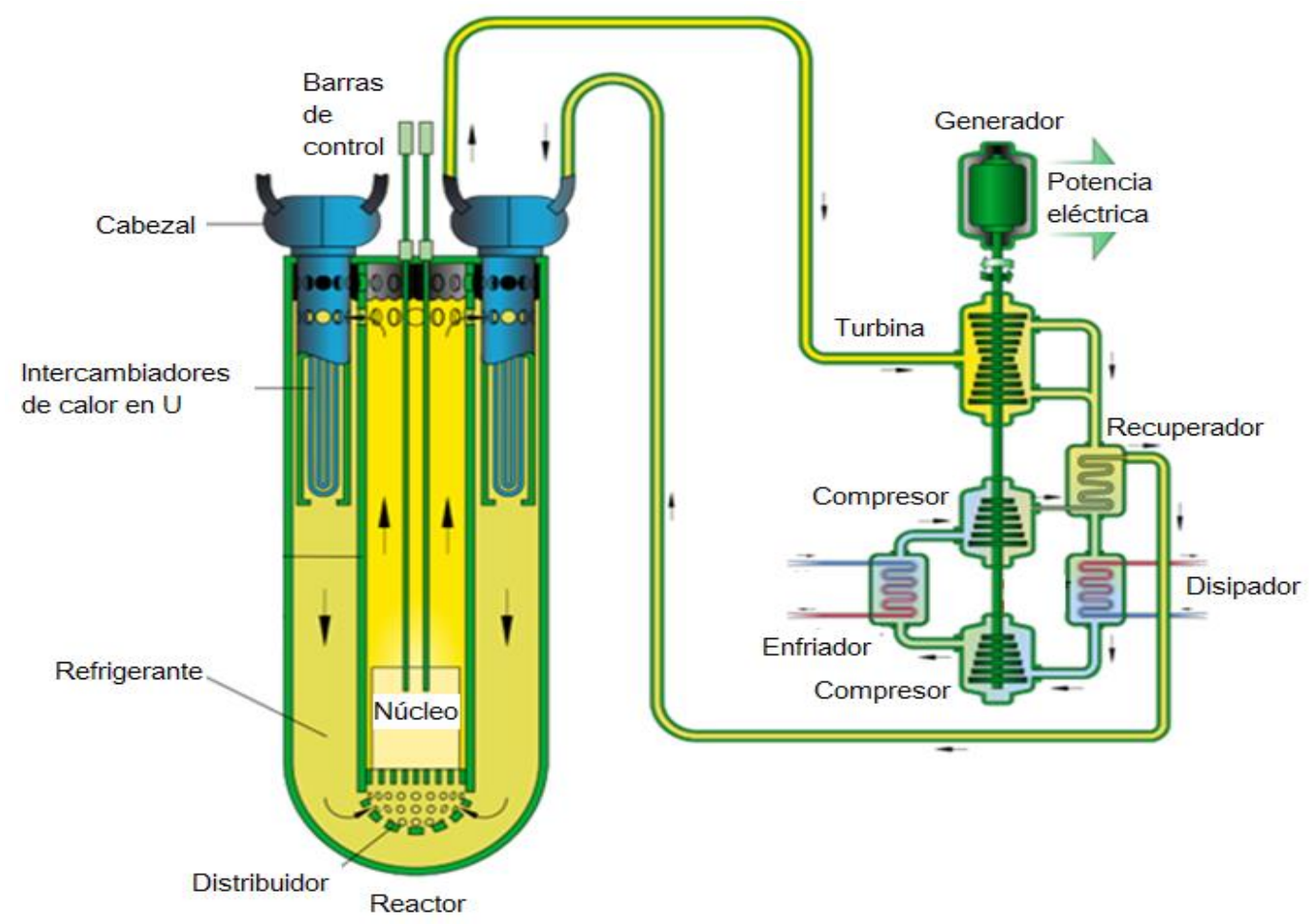

Figura 1.9. Esquema general del Reactor Rápido enfriado con plomo (GIF, 2016). 


\section{Reactor de Sal Fundida (MSR)}

El combustible del MSR (Molten Salt Reactor) (Figura 1.10) es el único que esta disuelto en el refrigerante, fluoruro de sodio. Es un tipo de reactor nuclear donde el refrigerante es una sal fundida. Han existido muchos diseños que han usado este concepto y se han construido unos pocos prototipos. Los primeros conceptos y muchos de los actuales se basan en un combustible nuclear disuelto en una sal de fluoruro fundida tal como el tetrafluoruro de uranio $\left(\mathrm{UF}_{4}\right)$ o tetrafluoruro de torio $\left(\mathrm{ThF}_{4}\right)$, el fluido alcanzaría criticidad al fluir en un núcleo de grafito que también serviría como el moderador. El sistema de reactor de sal fundida incorpora la característica muy especial de un combustible líquido.

Los MSR pueden utilizarse como quemadores de elementos transuránicos, también tienen una capacidad de reproducción en cualquier tipo de espectro de neutrones que va desde térmica (con un ciclo de combustible de torio) hasta rápida (ciclo del combustible uranio-plutonio), además de ser una tecnología considerable para la minimización de los residuos nucleares radiotóxicos.

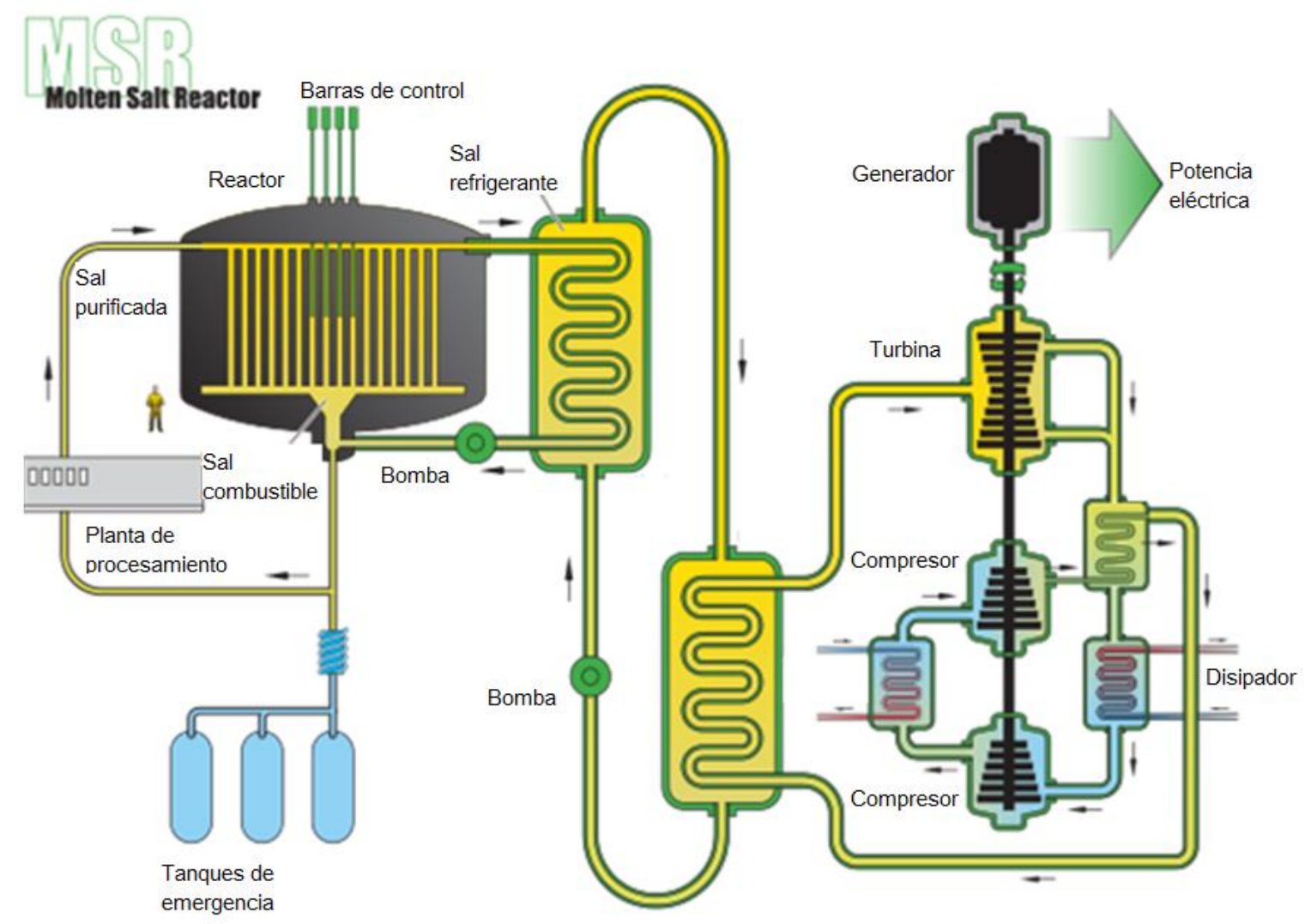

Figura 1.10. Esquema general del Reactor de Sal fundida (GIF, 2016). 


\section{Reactor Rápido Enfriado por Gas (Gas-Cooled Fast Reactor, GFR)}

El GFR (Figura 1.11) es un reactor de alta temperatura enfriado con helio y ciclo cerrado de combustible. Éste combina los avances de los sistemas de espectro rápido con los sistemas se alta temperatura. El usar un espectro rápido contribuye a un uso sustentable de las fuentes de uranio y a la minimización de los desechos a través del reciclado del combustible y el quemado de actínidos de larga vida y la temperatura alta provee la eficiencia de un ciclo térmico alto e industrial usado para generación de calor para procesos.

El GFR utiliza los mismos procesos de reciclaje de combustible que el SFR y la misma tecnología del reactor que el VHTR. Por lo tanto, su enfoque de desarrollo debe basarse, en la medida de lo posible, en tecnologías desarrolladas para el VHTR para estructuras, materiales, componentes y sistema de conversión de energía. Sin embargo, pide una investigación y desarrollo específicos más allá de los trabajos actuales y previstos sobre el sistema VHTR, principalmente sobre el diseño básico y el enfoque de seguridad.

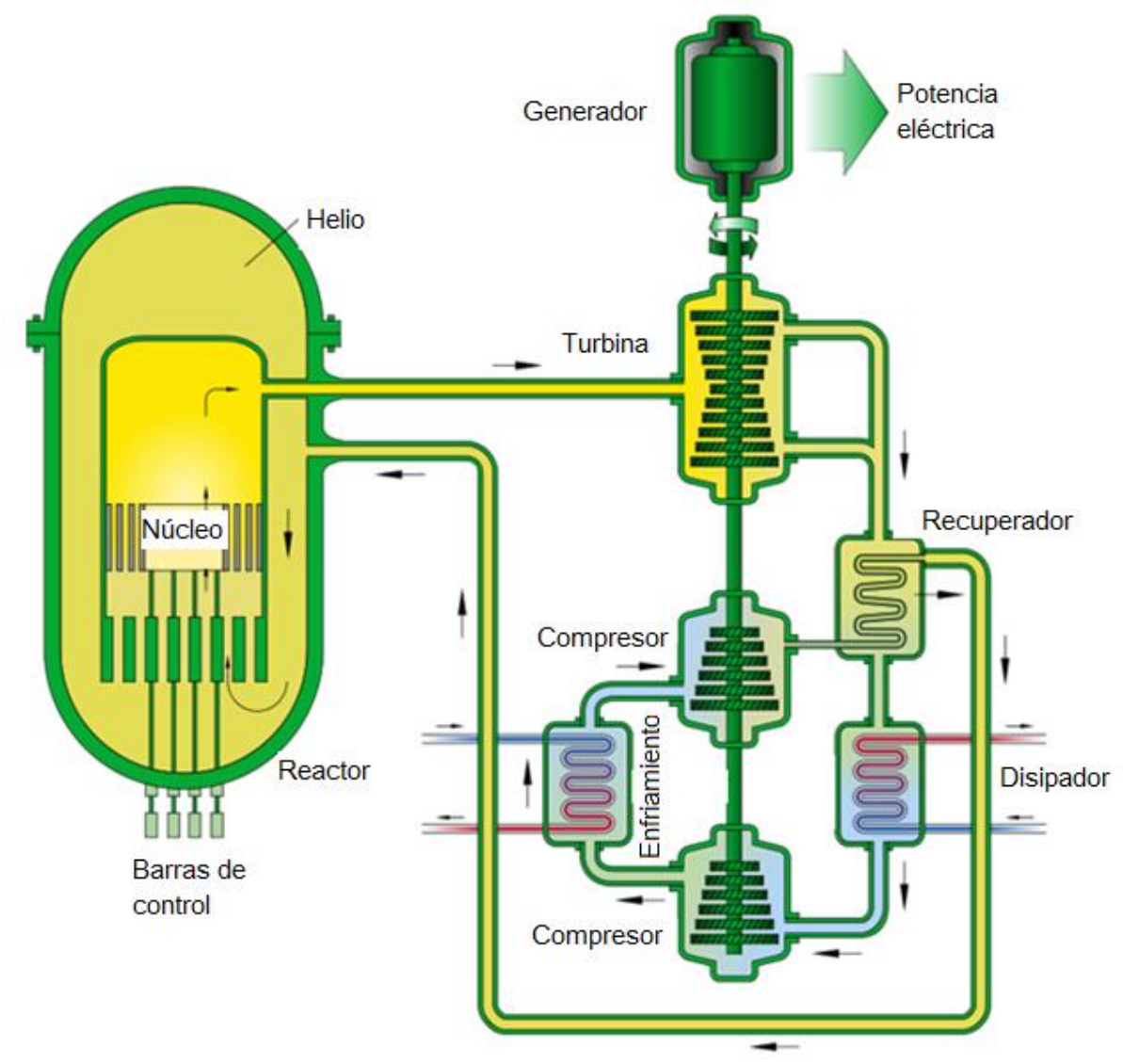

Figura 1.11. Esquema general del Reactor Rápido enfriado por Gas (GIF, 2016). 


\subsection{Los reactores nucleares y el desarrollo sustentable}

En 1987, el desarrollo sustentable fue presentado formalmente por la Comisión Mundial de Medio Ambiente y Desarrollo de la Organización de las Naciones Unidas, como una alternativa al desarrollo socioeconómico tradicional, causante de graves daños ambientales al planeta. Entre los factores clave del desarrollo sustentable, se encuentra el crecimiento poblacional, la demanda energética, el cambio climático, la escasez de recursos y del agua, y el manejo de residuos.

Los esfuerzos mundiales se han enfocado en promover y motivar a las poblaciones a dirigirse hacia un desarrollo sustentable utilizando una mezcla de fuentes de energía de forma que no se continúe con la dependencia de los combustibles fósiles.

En este sentido, los sistemas nucleares son una gran alternativa para el desarrollo sustentable, debido a que durante su operación no emiten gases de efecto invernadero y puede responder al aumento en la demanda de la energía. Es necesario construir centrales nucleares de diseño avanzado, que permitan aumentar la eficiencia y reducir los desechos, así como competir económicamente con otras tecnologías.

Dentro de los desarrollos de reactores nucleares, los reactores de Generación IV prometen cumplir con estas características, de modo que contribuyan en gran medida al desarrollo sustentable ya que los objetivos propuestos para estas tecnologías consideran la eficiencia del sistema, la competitividad económica, seguridad para la población y el entorno, manejo y disminución de los residuos y evitar la proliferación de armas nucleares.

El diseño de ciclo cerrado para los reactores rápidos promete disminuir en dos órdenes de magnitud el consumo de uranio natural, comparado con los reactores de agua ligera utilizados actualmente y en la misma magnitud disminuir los desechos finales (Artioli et al., 2010), esto evitaría la extracción del uranio y las consecuencias que de esta actividad se derivan así como la inversión y riesgo del manejo de los residuos. 


\section{Hipótesis y objetivos}

\subsection{Hipótesis}

Es posible modelar los procesos físicos presentes en un canal promedio del núcleo de un reactor rápido enfriado con plomo, a partir del acoplamiento de los procesos del termofluido, de transferencia de calor en el combustible y los procesos neutrónicos presentes.

\subsection{Objetivo general}

Analizar y modelar los procesos neutrónicos, de trasferencia de calor y de termofluido de un reactor rápido enfriado con plomo y realizar experimentación numérica que permita un análisis de seguridad nuclear.

\subsection{Objetivos específicos}

- Analizar las ecuaciones del termofluido y su efecto en los parámetros importantes del reactor rápido, como las temperaturas del combustible, la potencia térmica, el flujo del metal líquido y el flujo neutrónico.

- Construir una herramienta para el diseño y análisis de seguridad de reactores rápidos enfriados con plomo, con modelos matemáticos y numéricos.

- Realizar un análisis de sensibilidad paramétrica e incertidumbre con técnicas de simulación Monte Carlo, para determinar el alcance del diseño del reactor. 


\section{Reactor rápido enfriado con plomo}

El Foro Internacional de Generación IV identificó al Reactor rápido enfriado con plomo (LFR) como una buena opción tecnológica para generación de electricidad, producción de hidrógeno y manejo de actínidos en un ciclo cerrado de combustible. Una de las características más importantes en este rector es el refrigerante relativamente inerte, dicha característica significa ventajas en términos de seguridad, simplificación del sistema y un gran potencial económico.

Rusia (La unión Soviética y posteriormente la Federación de Rusia) tiene una gran experiencia industrial y operacional de reactores enfriados con plomo o plomo-bismuto. Iniciando con su programa de diseño y producción de reactores enfriados con plomo-bismuto usados para proveer energía a los submarinos desde la década de 1950 a la de los 90 . En este periodo se construyeron 12 reactores y 14 núcleos de reactor. En total, el funcionamiento de estos reactores dio como resultado 80 años-reactor de experiencia en operación.

Entre los 6 sistemas de Generación IV propuestos por el GIF para investigación y desarrollo, el Reactor rápido enfriado con plomo junto a los Reactores rápidos enfriados por sodio y por gas han sido seleccionados por la Unión Europea para enfocar su investigación. Estos sistemas son los mejores candidatos para tener un ciclo cerrado de combustible, asegurando una explotación de alta eficiencia de las fuentes de uranio así como la minimización de deshechos de larga vida (Grasso et al., 2013).

Dentro de los planes de investigación de la Unión Europea (Framework Programme, FP,) se han desarrollado los programas para Investigación y Desarrollo (I+D) de los reactores rápidos enfriados con plomo. Dentro del FP7 (Seventh Framework Programme) se inició el desarrollo del proyecto LEADER para I+D del LFR, este proyecto se basa en la experiencia obtenida con el diseño del reactor ELSY (European Lead System) desarrollado dentro del FP6 (Sixth Framework Programme). El proyecto LEADER contempla el desarrollo y construcción de dos reactores, un reactor de tamaño industrial llamado ELFR (European Lead-cooled Fast Reactor) de $1500 \mathrm{MW}_{\text {th }} \mathrm{y}$ el reactor ALFRED (Advanced Lead- cooled Fast Reactor) el cual es de tipo demostrativo de $300 \mathrm{MW}_{\text {th }}$ (Grasso et al., 2014).

En la Figura 3.1 se muestran los reactores enfriados con plomo que están considerados en el FP7 para su desarrollo y construcción, esta proyección se realizó a partir de 2010 con la experiencia de los programas anteriores y los reactores desarrollados en estos. De acuerdo al avance que se tiene en los reactores y los resultados se da una retroalimentación para los siguientes desarrollos. En este trabajo se toma como referencia el reactor ALFRED, actualmente está en etapa de desarrollo y se espera que para finales de la década de 2020 se pueda tener en operación. 


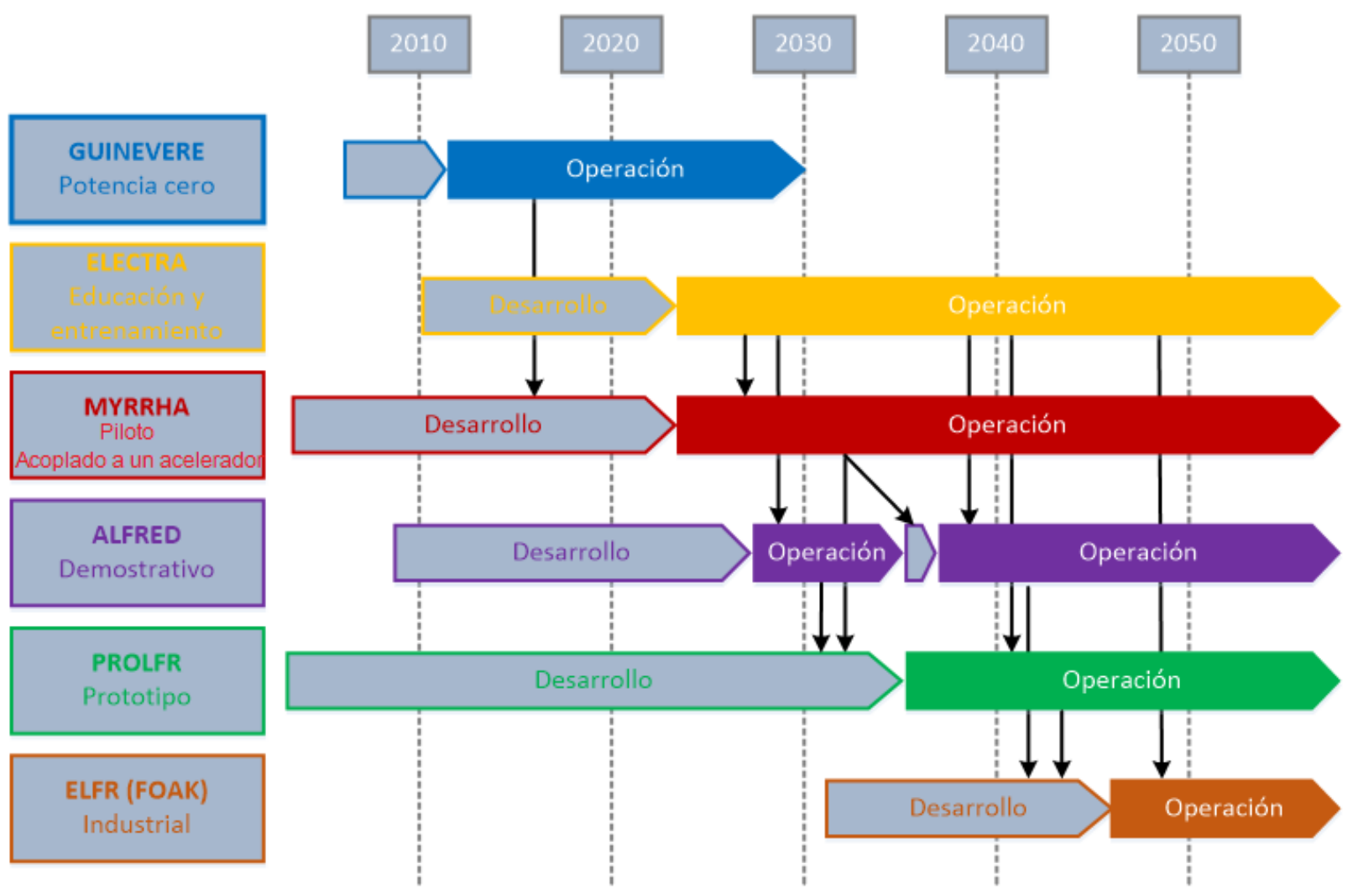

Figura 3.1. Reactores LFR programados dentro del FP7 de la Unión Europea (GIF, 2016).

\subsection{Conceptos básicos de un LFR}

Dentro de los sistemas nucleares de fisión existen los reactores térmicos y los reactores rápidos, los reactores rápidos pueden ser enfriados con metales líquidos (como sodio o plomo) o enfriados por gas (helio). Estos reactores tienen dos características principales:

- Los neutrones son de alta energía

- No utilizan moderador

En la Figura 3.2 se muestran las características principales de un reactor nuclear enfriado con plomo. Éste consta de un núcleo activo en el que se mantiene la cadena de fisiones y en donde se libera casi toda la energía de fisión en forma de calor. El núcleo del reactor contiene el combustible nuclear, este combustible contiene una mezcla de óxidos de Uranio y Plutonio (MOX). A través del núcleo circula el 
refrigerante, en este caso es el plomo, cuya función es extraer el calor generado por el núcleo, al salir de éste, el refrigerante entra a un generador de vapor, en donde como su nombre lo indica se genera el vapor que va hacia la turbina, este sistema evita la utilización de otro fluido entre el refrigerante y el vapor. Posteriormente la turbina de vapor envía trabajo mecánico al generador eléctrico y se produce la electricidad. Por otro lado, el vapor que sale de la turbina pasa por un sistema de condensado y vuelve a ingresar al generador de vapor.

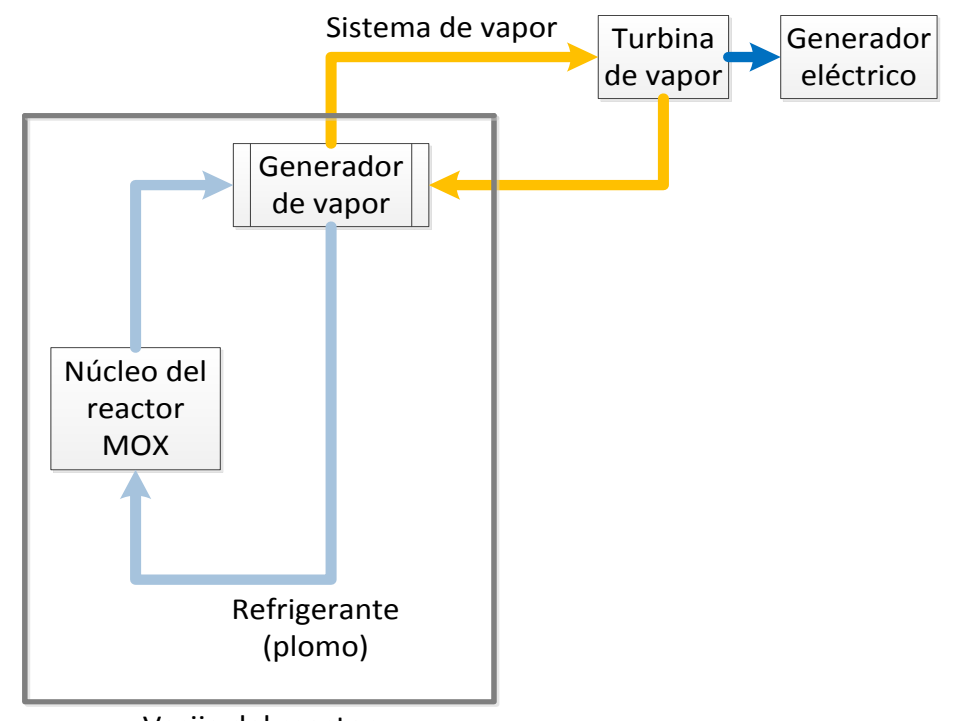

Vasija del reactor

Figura 3.2. Características principales de un LFR.

En la Figura 3.3 se muestra el proceso de fisión, el cual inicia con un neutrón que colisiona a un átomo de material fisionable (U-235, Pu-239), el neutrón es absorbido y el átomo es excitado, lo que ocasiona que se divida en dos fragmentos, liberando a su vez en promedio 2.5 neutrones y $200 \mathrm{MeV}$ de energía, los neutrones liberados son de alta energía (alrededor de $2.5 \mathrm{MeV}$ ) y se conocen como neutrones rápidos.

Para lograr la fisión de los átomos, la energía del neutrón absorbido depende de la naturaleza del átomo fisionable, para el U-235 se requieren neutrones térmicos, los cuales tienen una energía menor de 0.025 eV y para el Pu-239 se requieren neutrones rápidos y térmicos (Smith, 2010). 

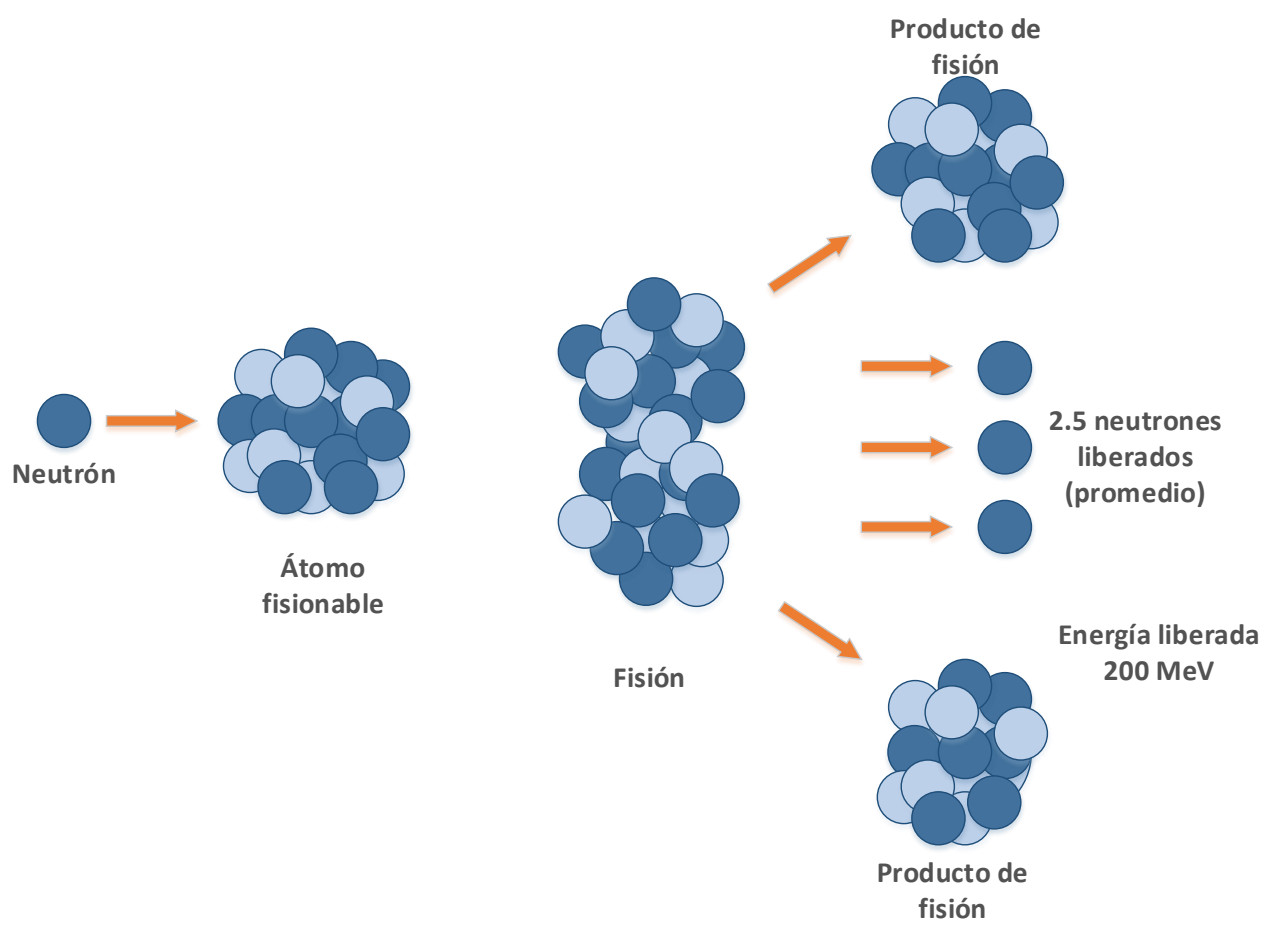

Figura 3.3. Reacción de fisión nuclear.

A continuación se muestran las reacciones de fisión y la energía que liberan, en el caso del Torio-232 y el Uranio-238 primero se absorbe un neutrón, generándose un átomo fisionable, el cual absorbe otro neutrón y se logra la reacción de fisión (ININ, 2014).

Las reacciones de fisión son:

- Uranio-235

${ }^{235} \mathrm{U}+1 \mathrm{n} \rightarrow$ Productos de Fisión $+2.4 \mathrm{n}+192.9 \mathrm{MeV}$

- Uranio-233 y Torio-232

${ }^{232} \mathrm{Th}+1 \mathrm{n} \rightarrow{ }^{233} \mathrm{U}+1 \mathrm{n} \rightarrow$ Productos de Fisión $+200 \mathrm{MeV}$

- Uranio-238

${ }^{238} \mathrm{U}+1 \mathrm{n} \rightarrow{ }^{239} \mathrm{U} \rightarrow{ }^{239} \mathrm{~Np} \sim>\beta \rightarrow{ }^{239} \mathrm{Pu} \sim>\beta^{-}+1 \mathrm{n} \rightarrow$ Productos de Fisión $+2.9 \mathrm{n}+198.5 \mathrm{MeV}$

- Plutonio-239

${ }^{239} \mathrm{Pu}+1 \mathrm{n} \rightarrow$ Productos de Fisión $+2.9 \mathrm{n}+198.5 \mathrm{MeV}$ 


\subsection{Descripción del reactor ALFRED}

Como se mencionó anteriormente ALFRED (Advanced Lead-cooled Fast Reactor European Demonstrator) es un reactor rápido enfriado con plomo desarrollado por la Unión Europea dentro del FP7. En esta sección se realizará la descripción de las características principales del reactor, partiendo del núcleo, ya que es la parte con mayor interés dentro de este trabajo. (Grasso et al., 2014)

En la Tabla 3.1 se muestran las características principales de operación de ALFRED, este reactor se considera pequeño, ya que su potencia térmica es de $300 \mathrm{MW}$, aproximadamente $125 \mathrm{MW}$ eléctricos.

Tabla 3.1. Características del reactor ALFRED (Grasso et al., 2013)

\begin{tabular}{lcc}
\hline \multicolumn{1}{c}{ Parámetro } & Unidad & Valor \\
\hline Potencia térmica & $\mathrm{MW}$ & 300 \\
Máximo radio interno de la vasija & $\mathrm{cm}$ & 170 \\
Tipo de ensamble & - & Hexagonal cerrado \\
Tipo de combustible & - & $\mathrm{MOX}$ \\
Enriquecimiento máximo de Pu & $\%$ & 30 \\
Temperatura máxima del combustible & ${ }^{\circ} \mathrm{C}$ & 2000 \\
Presión máxima & $\mathrm{MPa}$ & 5 \\
Material del encamisado & - & $15-15 \mathrm{Ti}$ \\
Temperatura máxima del encamisado & ${ }^{\circ} \mathrm{C}$ & 550 \\
Refrigerante & - & $\mathrm{Plomo}$ \\
Temperatura de entrada del refrigerante & ${ }^{\circ} \mathrm{C}$ & 400 \\
Temperatura de salida del refrigerante & ${ }^{\circ} \mathrm{C}$ & 480 \\
Velocidad máxima del refrigerante & $\mathrm{m} / \mathrm{s}$ & 3 \\
\hline
\end{tabular}

El tipo de ensamble es hexagonal, cada ensamble cuenta con 127 barras de combustible (Figura 3.4.) y en total en el núcleo se tienen 171 ensambles. La potencia térmica por barra es de $13.81 \mathrm{~kW}$ (Tabla 3.2).

Las dimensiones de la barra de combustible se muestran en la Figura 3.5, dentro de la barra se tiene la región activa, que es en donde se encuentra el combustible. En la sección transversal de la barra se tienen la sección de combustible (MOX), el gap que es la zona que evita que el combustible toque la pared de la barra y es un espacio que contiene gas, en este caso es helio, y la sección de encamisado, la cual envuelve el combustible y permite la transferencia de calor hacia el refrigerante. 


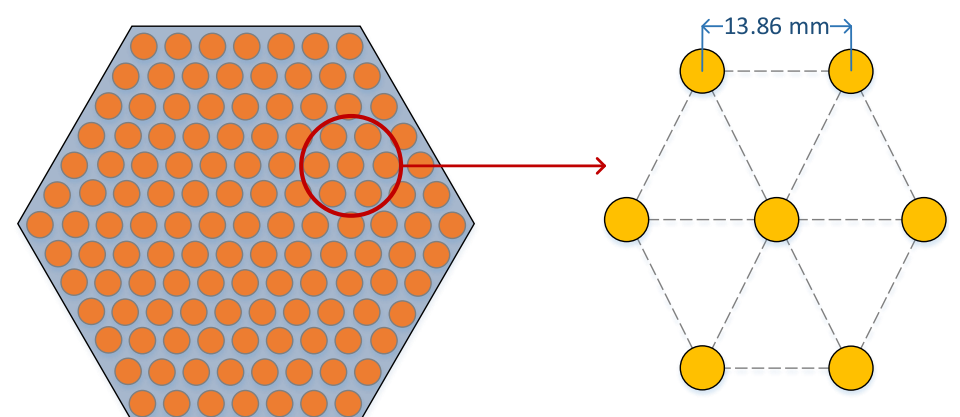

Figura 3.4. Ensamble hexagonal.

Tabla 3.2. Características del núcleo.

\begin{tabular}{lcc}
\hline \multicolumn{1}{c}{ Parámetro } & Unidad & Valor \\
\hline Número de ensambles & - & 171 \\
Barras de combustible por ensamble & - & 127 \\
Potencia térmica por barra & KW/barra & 13.81 \\
Distancia entre barras de combustible & $\mathrm{mm}$ & 13.86 \\
\hline
\end{tabular}

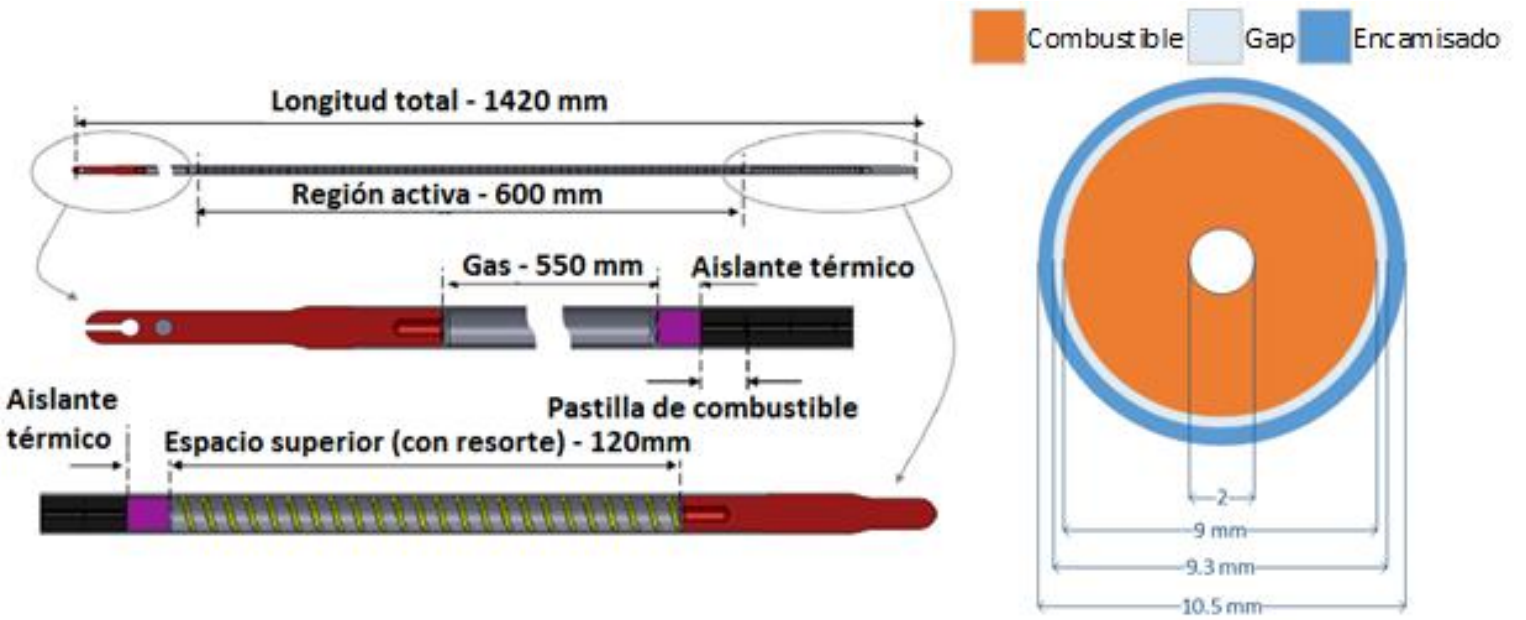

Figura 3.5. Barra de combustible del reactor ALFRED (izquierda), sección transversal de una barra de combustible (derecha) [Modificado de (Grasso et al., 2014)].

En el núcleo del reactor la distribución de ensambles de combustible se divide en zona interna y externa de acuerdo al porcentaje de enriquecimiento del combustible que tienen (Figura 3.6), la zona interna tiene 57 ensambles de combustible con un enriquecimiento de $21.7 \%\left(\mathrm{Pu}+{ }^{241} \mathrm{Am}\right) /\left(\mathrm{Pu}+{ }^{241} \mathrm{Am}+\mathrm{U}\right)$, y los restantes 114 ensambles constituyen la zona externa con 27.8\% (Grasso et al., 2014).

La configuración del núcleo del reactor considera la posición de las barras de control y las barras de seguridad. Para este modelo se tienen 16 barras de control y 4 barras de seguridad, el material absorbente considerado para estas barra es el $\mathrm{B}_{4} \mathrm{C}$ (con $90 \%$ de ${ }^{10} \mathrm{~B}$ ) y una densidad de $2.2 \mathrm{~g} / \mathrm{cm}^{3}$. 


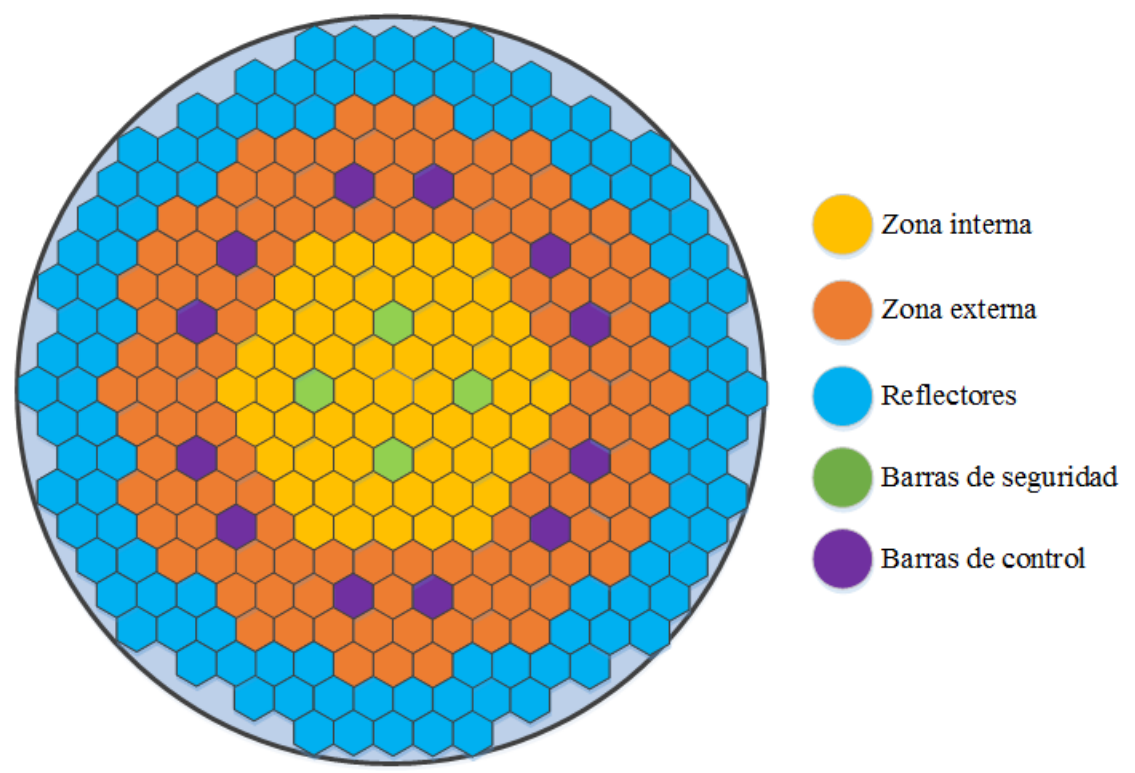

Figura 3.6. Configuración del núcleo del reactor ALFRED [Adaptado de (Grasso et al., 2014)].

Las barras de control permiten encender y apagar el reactor, mantener controlada la reactividad durante el ciclo de combustible y el ajuste de la potencia. Las barras de seguridad solo son usadas para casos de SCRAM (Grasso et al., 2013).

Las barras de control son enfriadas con el plomo, son accionadas mediante un motor durante la operación del reactor, para emergencias de falta de energía las barras funcionan con una conexión electromagnética que permite la rápida inserción dentro del núcleo. Las barras de control están provistas de un mecanismo guía para asegurar una segura inserción dentro del núcleo en caso de distorsión del sistema, como en caso de sismos.

Las barras de seguridad también son enfriadas por el plomo, durante la operación normal del reactor se encuentran arriba de la zona activa. Su única operación es en caso de SCRAM, cuando es necesario un sistema electromagnético las inserta rápidamente dentro del núcleo. 


\section{Propiedades físicas}

\subsection{Propiedades del combustible, gap y encamisado}

En esta sección se describirán las propiedades principales de los componentes de una barra de combustible en su vista transversal. Las barras tienen tres componentes principales; MOX (mezcla de óxidos), Helio (He) y T91. A continuación se muestran las correlaciones que determinan las propiedades del MOX (Carbajo et al., 2001). La densidad del MOX está en función de la temperatura, pero para poder calcularla es necesaria calcular la densidad a $273 \mathrm{~K}$, con la siguiente ecuación se puede calcular la densidad de la mezcla de óxidos, considerando a y como la fracción molar de $\mathrm{PuO}_{2}$.

$$
\rho_{\text {MOX }}(273)=10970+490 \cdot y
$$

Con la densidad de referencia de $273 \mathrm{~K}$ es posible calcular la densidad en función de la temperatura de acuerdo a la siguiente ecuación

$$
\rho_{M O X}=\frac{\rho_{M O X}(273)}{\left(9.9672 \times 10^{-1}+1.179 \times 10^{-5} T-2.429 \times 10^{-9} T^{2}+-1.219 \times 10^{-12} T^{3}\right)^{3}}
$$

La ecuación de la capacidad calorífica está dada por

$$
C p_{\mathrm{MOX}}=(1-y) C p_{\mathrm{UO}_{2}}+y C p_{\mathrm{PuO}_{2}}
$$

En donde es necesario conocer la capacidad calorífica del $\mathrm{UO}_{2}$ y del $\mathrm{PuO}$, así como la fracción molar del óxido de plutonio. Para calcular el $C p$ de cada uno se utiliza la ecuación (4.4). En la Tabla 4.1 se muestran los valores de $C_{1}, C_{2}, C_{3}, \theta, E_{a}$ para $U O_{2}$ y del $P u O_{2}$.

$$
C p=\frac{C_{1} \theta^{2} e^{\frac{\theta}{T}}}{T^{2}\left(e^{\frac{\theta}{T}}-1\right)}+2 C_{2} T+\frac{C_{3} E_{a} e^{-\frac{E_{a}}{T}}}{T^{2}}
$$


Tabla 4.1. Constantes para el cálculo de la densidad (Carbajo et al., 2001).

\begin{tabular}{cccc}
\hline Constate & $\mathrm{UO}_{2}$ & $\mathrm{PuO}_{2}$ & Unidades \\
\hline$c_{1}$ & 302.27 & 322.49 & $\mathrm{KJ} / \mathrm{kg} / \mathrm{K}$ \\
$c_{2}$ & $8.463 \times 10^{-3}$ & $1.4679 \times 10^{-2}$ & $\mathrm{~J} / \mathrm{kg} / \mathrm{K}^{2}$ \\
$c_{3}$ & $8.7421 \times 10^{7}$ & 0 & $\mathrm{~J} / \mathrm{Kg}$ \\
$\theta$ & 548.68 & 587.41 & $\mathrm{~K}$ \\
$E_{a}$ & 18541.7 & 0 & $\mathrm{~K}$ \\
\hline
\end{tabular}

Finalmente para la conductividad térmica se tiene la ecuación (4.5)

$$
k_{M O X}=1.158\left[\frac{1}{A+C \tau}+\frac{6400 e^{\frac{-16.35}{\tau}}}{\tau^{\frac{5}{2}}}\right]
$$

En donde cada variable esta descrita por una correlación, en donde $x$ es la desviación estándar de la estequiometria. En este caso la desviación es $x=0.03$ (Carbajo et al., 2001).

$$
A=2.85 x+0.035 \quad C=-0.715 x+0.286 \quad \tau=\frac{T}{1000}
$$

El Helio es el gas que se encuentra en el gap y en el huelgo que tiene la pastilla de combustible, este evita que la pastilla toque el encamisado y permite la dilatación de las pastillas. Las propiedades requeridas del He para el presente trabajo son la densidad $(\rho)$, la capacidad calorífica $(C p)$ y la conductividad térmica $(k)$. En la Tabla 4.2 se muestran los valores de estas propiedades, para la conductividad térmica se cuenta con una correlación en función de la temperatura.

Tabla 4.2. Propiedades del Helio (Carbajo et al., 2001).

\begin{tabular}{lcc}
\hline \multicolumn{1}{c}{ Propiedad } & Valor/correlación & Unidades \\
\hline Densidad & 2.425 & $g / \mathrm{m}^{3}$ \\
Capacidad calorífica & 5191.0 & $J /(K \mathrm{~kg})$ \\
Conductividad térmica & $k=15.8 \times 10^{-4} T^{0.7}$ & $W /(K \mathrm{~m})$ \\
\hline
\end{tabular}


El diseño de nuevas generaciones de reactores nucleares incluye la selección del encamisado, este debe ser un material que cubra las necesidades de transferencia de calor y resistencia. El acero con T91 es utilizado como encamisado de las barras de combustible y constantemente está en mejoras para satisfacer las necesidades de las futuras generaciones de reactores.

Tabla 4.3. Propiedades del acero T91 (Carbajo et al., 2001).

\begin{tabular}{lcc}
\hline \multicolumn{1}{c}{ Propiedad } & Valor & Unidades \\
\hline Densidad & 7700.0 & $g / \mathrm{m}^{3}$ \\
Capacidad calorífica & 622.0 & $J /(K \mathrm{~kg})$ \\
Conductividad térmica & 26.0 & $W /(K \mathrm{~m})$ \\
\hline
\end{tabular}

\subsection{Propiedades del plomo}

Los metales líquidos como el sodio, plomo y la mezcla eutéctica de plomo-bismuto son considerados refrigerantes potenciales para reactores nucleares rápidos, estos han sido considerados desde el inicio de la tecnología nuclear para su uso, es por eso que el conocimiento de las propiedades de estos metales es de gran importancia para el diseño y operación de los reactores.

Los criterios que se deben considerar para elegir un metal líquido como refrigerante se enumeran a continuación (Campos et al., 2015):

1. Propiedades de la transferencia de calor: El refrigerante debe dar altos coeficientes de transferencia de calor y debe poseer una capacidad calorífica razonablemente alta.

2. Corrosión y actividad química: El líquido refrigerante debe tener baja afinidad química hacia las paredes de su contenedor, las barras de combustible y en general hacia todas las superficies sólidas con que entra en contacto, así como debe tener una inercia química con los fluidos de trabajo si se presentan fugas.

3. Punto de ebullición: el punto normal de ebullición del refrigerante debe estar muy por encima del rango de temperaturas en que opera. Esto conservará las propiedades del refrigerante líquido aun operando a altas temperaturas.

4. Descomposición: El refrigerante debe ser estable en un ambiente de alta temperatura y radiación. 
5. Punto de fusión: En ocasiones se requiere de un sistema de precalentamiento para mantener el refrigerante en estado líquido, por lo que se prefiere un punto de fusión mucho más bajo.

6. Reactividad: Lo ideal es que el refrigerante no tenga actividad de radiación gamma en la vasija. En el caso de que la tenga, se busca que la vida media de estas actividades sea corta y de baja energía.

7. Propiedades nucleares: las propiedades nucleares del refrigerante son muy importantes ya que normalmente ocupa más del 30\% del volumen del núcleo del reactor. La capacidad del moderador debe ser baja. Es deseable que el coeficiente de reactividad por temperatura sea negativo. El líquido refrigerante debe tener bajos valores de sección eficaz de absorción y dispersión inelástica.

8. Costo: El costo de inversión del metal líquido refrigerante puede ser importante, sin embargo, es sólo una pequeña fracción de la inversión total de la planta nuclear.

Es necesario saber que por ahora ningún refrigerante cumple perfectamente estos criterios. Los puntos de 1 a 4 y el 7 son los más importantes para elegir a un refrigerante primario, aunque otros refrigerantes pueden ser opción si los requisitos para el reactor no son tan estrictos, por ejemplo, si la densidad de potencia es suficientemente baja.

Campos y Osornio en su reporte de Servicio Social de la Universidad Autónoma Metropolitana en 2015 realizan una compilación de las propiedades fundamentales del plomo a presión atmosférica estándar $(0.1$ $\mathrm{MPa}$ ), a continuación se muestran las correlaciones que describen cada una de las propiedades.

\section{Temperatura de fusión}

El punto de fusión es la temperatura a la cual se encuentra el equilibrio de fases sólido-líquido, es decir la materia pasa de estado sólido a estado líquido, se funde. Cabe destacar que el cambio de fase ocurre a temperatura constante. El punto de fusión es una propiedad intensiva.

$$
T_{M, 0(P b)}[K]=600.6 \pm 0.1
$$

El punto de fusión del plomo incrementa en $0.0792 \mathrm{~K}$ por $1 \mathrm{MPa}$, cuando la presión aumenta de 15 a 200 $\mathrm{MPa}$. El incremento continua en menor medida; $0.0671 \mathrm{~K}$ por $1 \mathrm{MPa}$, en el rango de $800-1200 \mathrm{MPa}$, y disminuye en $5.4 \mathrm{~K}$ cuando la presión aumenta de 2 a 3 GPa. 


\section{Calor latente de fusión}

Se llama calor de fusión a la energía necesaria para cambiar 1 gramo de sustancia en estado sólido a estado líquido, sin cambiar su temperatura. Esta energía rompe los enlaces de sólidos, y queda una significativa cantidad, asociada con las fuerzas intermoleculares del estado líquido.

$$
\Delta H_{M, 0(P b)}=4.78 \pm 0.03\left[\mathrm{~kJ} \cdot \mathrm{mol}^{-1}\right]=23.07 \pm 0.14\left[\mathrm{~kJ}^{\mathrm{kg}} \mathrm{kg}^{-1}\right]
$$

El valor señalado para el calor latente del plomo se encuentra en el rango de $4.76-5.11 \mathrm{~kJ} / \mathrm{mol}, \sim 10^{5} \mathrm{~Pa}$.

\section{Temperatura de ebullición}

La Temperatura de ebullición se define como la temperatura a la cual la presión de vapor del líquido es igual a una atmósfera. El punto de ebullición aumenta cuando se aplica presión. En este punto el líquido pasa de fase líquida a gaseosa.

$$
T_{B, 0(P b)}[K]=2021 \pm 3
$$

El cambio en la temperatura de ebullición del plomo es $14 \mathrm{~K}$, a una presión con un orden de magnitud de $10^{5} \mathrm{~Pa}$.

\section{Calor latente de ebullición}

El calor latente es la energía requerida por una cantidad de sustancia para cambiar de fase de líquido a gaseoso. Se debe tener en cuenta que esta energía en forma de calor se invierte para el cambio de fase y no para un aumento de la temperatura.

$$
\Delta H_{B, 0(P b)}=177.9 \pm 0.4\left[\mathrm{~kJ} \cdot \mathrm{mol}^{-1}\right]=858.6 \pm 1.9\left[\mathrm{~kJ} \cdot \mathrm{kg}^{-1}\right]
$$

Este valor presenta una variación de $1 \%$ entre el valor máximo y mínimo, a una presión con un orden de magnitud de $10^{5} \mathrm{~Pa}$.

\section{Punto crítico}

Es el punto al cual los estados de líquido saturado y de vapor saturado son idénticos. La temperatura, la presión y la densidad específica de una sustancia en el punto crítico se denominan, temperatura crítica, presión crítica y densidad específica crítica. 


$$
\begin{gathered}
T_{c(P b)}[K]=5000 \pm 200 \\
\rho_{c(P b)}\left[\mathrm{kg} \cdot \mathrm{m}^{-3}\right]=3250 \pm 100 \\
p_{c(P b)}[\mathrm{MPa}]=180 \pm 30
\end{gathered}
$$

\section{Densidad}

La densidad se define como la cantidad de masa contenida en un volumen, se considera variable en función de la temperatura y la presión. En este caso se presenta la correlación en función de la temperatura a una presión constante con un orden de magnitud de $10^{5} \mathrm{~Pa}$. (Figura 4.1.)

$$
\rho_{(P b)}\left[\mathrm{kg} \cdot \mathrm{m}^{-3}\right]=11441-1.2795 \cdot T
$$

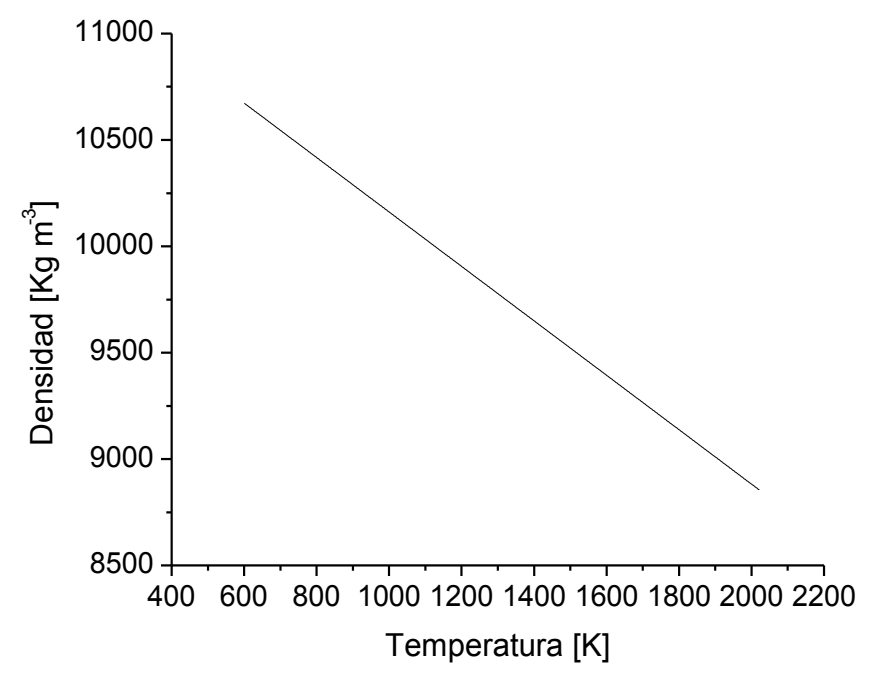

Figura 4.1. Perfil de la densidad del plomo a diferentes temperaturas (Campos et al., 2015).

\section{Coeficiente de expansión térmica}

El coeficiente de dilatación (o más específicamente, el coeficiente de expansión térmica) es el cociente que mide el cambio relativo de longitud o volumen que se produce cuando un cuerpo sólido o un fluido 
cambia de temperatura provocando una dilatación térmica. Generalmente se denota por $\alpha$ y sus unidades en el SI son $\left[K^{-1}\right]$. El comportamiento del coeficiente de expansión se presenta en la Figura 4.2.

$$
\alpha_{p}\left(T, p_{0}\right)=\frac{1}{\frac{\rho_{M, 0}}{A_{\rho, 0}}+T_{M, 0}-T}
$$

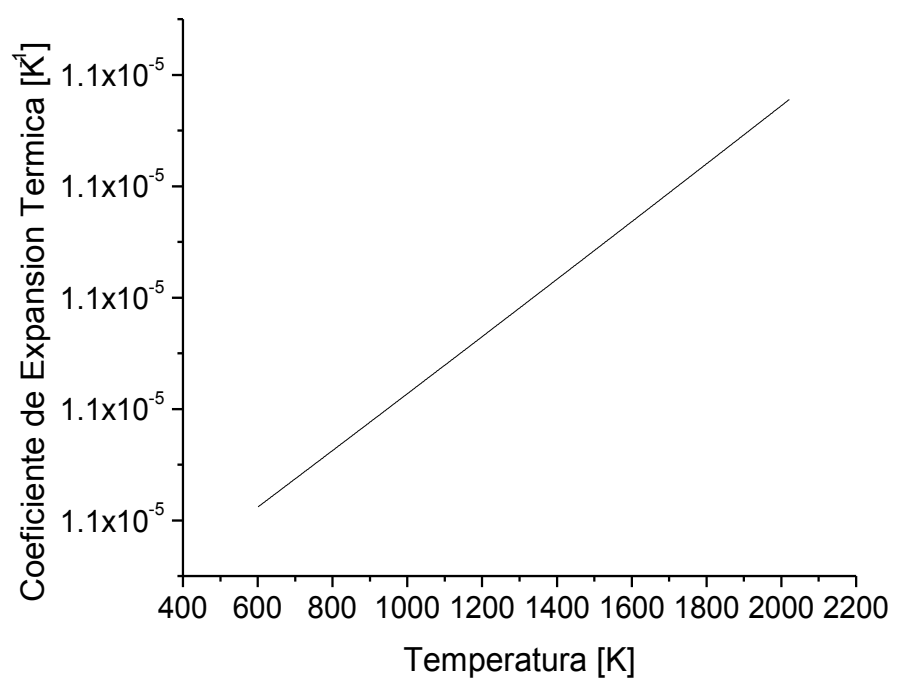

Figura 4.2. Perfil del coeficiente de expansión del plomo a diferentes temperaturas (Campos et al., 2015).

\section{Capacidad calorífica}

La capacidad calorífica o capacidad térmica de un cuerpo es el cociente entre la cantidad de energía calorífica transferida a un cuerpo o sistema en un proceso cualquiera y el cambio de temperatura que experimenta. La conductividad térmica se denota por $C p$ cuando es a presión constante y sus unidades en el SI son $\left[J \cdot \mathrm{kg}^{-1} \cdot K^{-1}\right]$. En la Figura 4.3 se muestra el comportamiento de ésta propiedad.

$$
C_{p(P b)}=175.1-4.961 \times 10^{-2} T+1.985 \times 10^{-5} T^{2}-2.099 \times 10^{-9} T^{3}-1.524 \times 10^{6} T^{-2}
$$




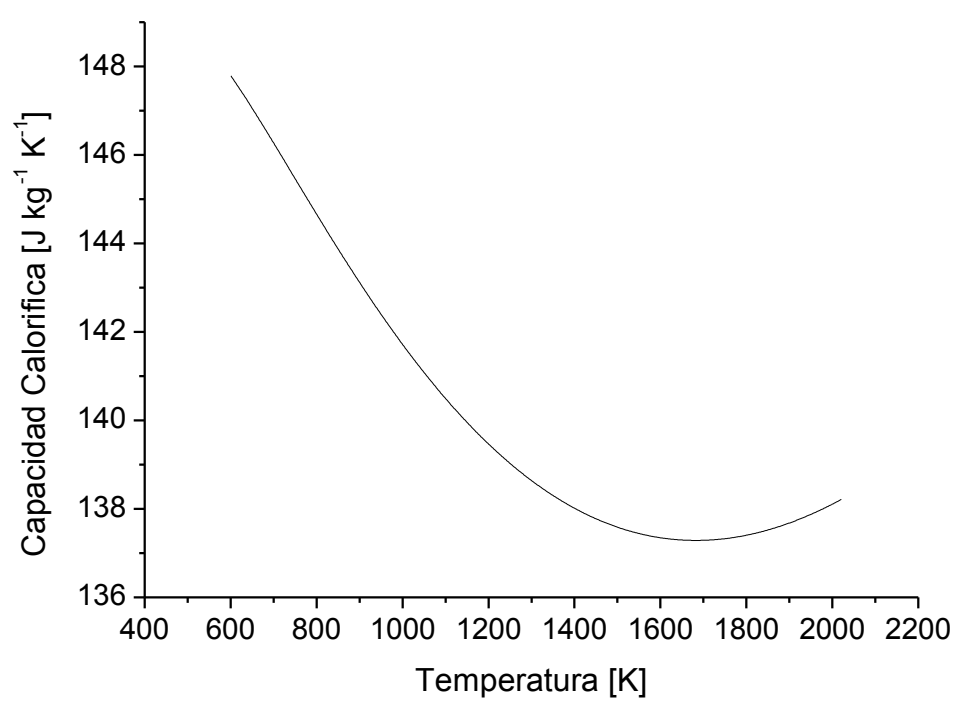

Figura 4.3. Perfil de la capacidad calorífica del plomo a diferentes temperaturas (Campos et al., 2015).

\section{Entalpía}

La entalpía expresa una medida de la cantidad de energía absorbida o cedida por un sistema termodinámico, es decir, la cantidad de energía que un sistema intercambia con su entorno. Sus unidades son $\left[J \cdot \mathrm{kg}^{-1}\right]$ y se denota por $H$. Para el plomo, en la Figura 4.4 se muestra el comportamiento de la entalpía.

$$
\begin{gathered}
H(T, p)=H\left(T_{M, 0}, p\right)+\int_{T_{M}}^{T} C_{p}(T, p) d T \\
\Delta H_{(P b)}\left(T_{M, 0(P b)}, T, p_{0}\right)=176.2\left(T-T_{M, 0(P b)}\right)-2.4615 \times 10^{-2}\left(T^{2}-T_{\left.M, 0(P b)^{2}\right)}\right. \\
+5.147 \times 10^{-6}\left(T^{3}-T_{M, 0(P b)^{3}}\right)+1.524 \times 10^{6}\left(T^{-1}-T_{M, 0(P b)^{-1}}\right)
\end{gathered}
$$




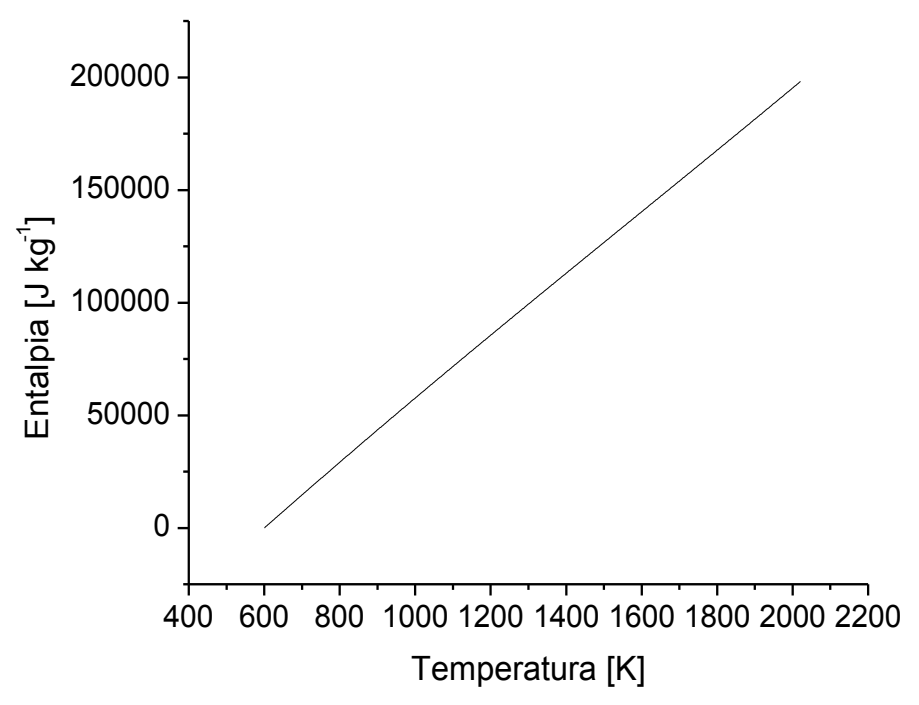

Figura 4.4. Perfil de la entalpía del plomo a diferentes temperaturas (Campos et al., 2015).

\section{Viscosidad}

La viscosidad es la resistencia que tienen las moléculas que conforman un líquido para separarse unas de otras, es decir, es la oposición de un fluido a deformarse y esta oposición es debida a las fuerzas de adherencia que tienen unas moléculas de un líquido o fluido con respecto a las otras moléculas del mismo líquido. En el SI sus unidades son $[P a \cdot s]$ y se denota como $\eta$ (Figura 4.5).

$$
\eta_{(P b)}=4.55 \times 10^{-4} \exp \left(\frac{1069}{T}\right)
$$

\section{Conductividad térmica}

La conductividad térmica se refiere a la cantidad/velocidad de calor transmitida a través de un material. La transferencia de calor se produce en mayor proporción en los materiales con alta conductividad térmica con respecto a aquellos con baja conductividad térmica. Se denota como $k$ y sus unidades son $\left[W \cdot m^{-1} \cdot K^{-1}\right]$ (Figura 4.6).

$$
k_{(P b)}=9.2+0.011 \cdot T
$$




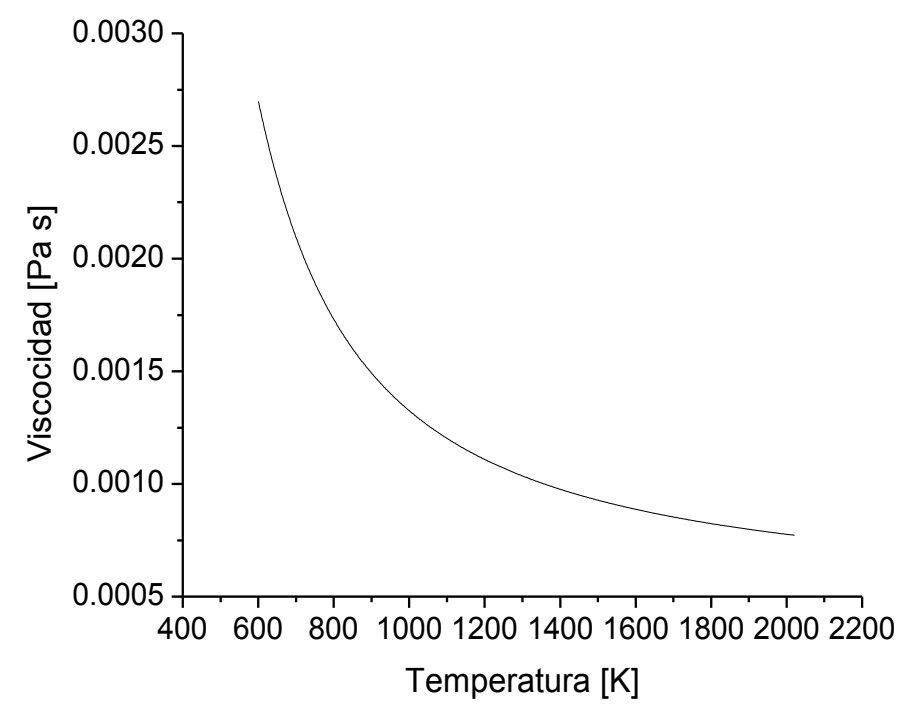

Figura 4.5. Perfil de la viscosidad del plomo a diferentes temperaturas (Campos et al., 2015).

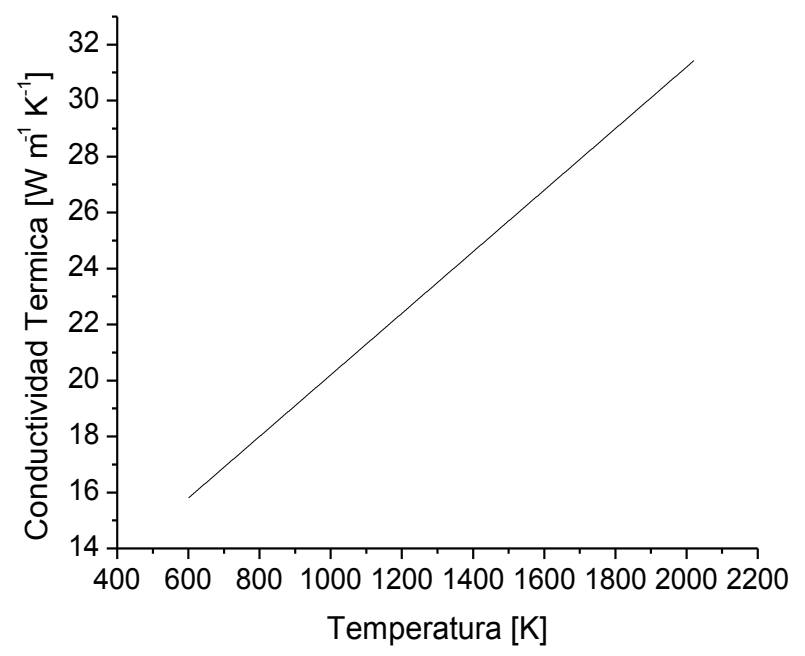

Figura 4.6. Perfil de la conductividad térmica del plomo a diferentes temperaturas (Campos et al., 2015). 


\section{Análisis del reactor}

En la investigación de los reactores nucleares existen dos áreas importantes, el diseño del núcleo del reactor y la gestión del combustible, así como el análisis de la seguridad de la planta. La primera se basa en la física del reactor o procesos neutrónicos y la segunda en análisis termohidráulico o termofluido. Ambas áreas se relacionan a través de la generación y transferencia de calor en el combustible y su transferencia al refrigerante (Barragán, 2013).

En este capítulo se describirá el modelo matemático utilizado para describir cada uno de los procesos analizados, los parámetros utilizados en cada caso y el método de solución, en primer lugar se analiza la parte neutrónica a partir del modelo puntual de la cinética neutrónica. Posteriormente el proceso de transferencia de calor en el combustible a partir de la conducción de calor y finalmente el análisis del plomo como refrigerante, en este último se realizan balances de masa, cantidad de movimiento y energía.

\subsection{Análisis de los procesos neutrónicos}

\subsubsection{Modelo puntual de la Cinética Neutrónica}

Existen tres factores que determinan la potencia de un reactor nuclear; la masa de material físil presente, la sección eficaz macroscópica de fisión y el flujo neutrónico, éste último es un factor con el cual se puede tener un control de dicha potencia.

Para describir la cinética neutrónica en función del tiempo en el LFR se utilizará la teoría de un grupo, es decir, se supone que el proceso de producción, difusión y absorción de neutrones se lleva a cabo con una sola energía y que en las colisiones de dispersión no se producen variaciones energéticas.

La potencia térmica del reactor está relacionada directamente con la densidad neutrónica por la ecuación (5.1), esta relación se lleva a cabo en cada barra de combustible, la densidad neutrónica es la cantidad de neutrones por $\mathrm{cm}^{3}$ que se generan en el reactor nuclear.

$$
P(z, t)=P_{o} n(t) \psi(z)
$$

donde

$P=$ potencia térmica promedio por barra

$P_{o}=$ potencia térmica nominal por barra 
$n(t)=$ densidad neutrónica

$\psi(z)=$ distribución de potencia axial

La $P_{o}$ se indica en la tabla 3.2, ésta fue calculada dividendo la potencia térmica nominal de reactor entre el número total de barras, considerando que todas las barras tienen el mismo enriquecimiento.

La distribución de potencia axial utilizada en este trabajo se muestra en la Figura 5.1 (Aufiero, 2013), se considera que la distribución es la misma para todas las secciones del núcleo del reactor.

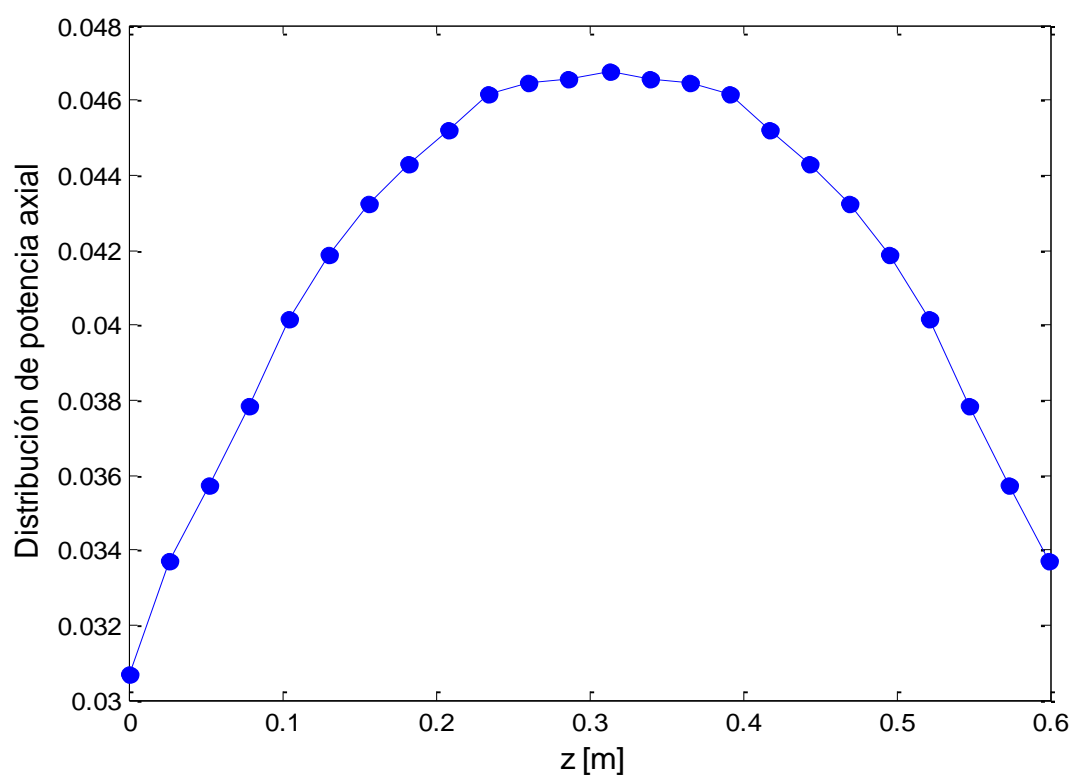

Figura 5.1.Distribución de potencia axial en la barra de combustible.

El modelo matemático para el análisis de la cinética neutrónica parte de una aproximación de la Teoría de difusión.

Teoría de difusión. La difusión se refiere al desplazamiento neto que tienen los neutrones desde regiones de mayor densidad neutrónica a otras de densidad menor (Glasstone y Sesonske, 1990). La ecuación de difusión considera la producción de neutrones por fisión, la pérdida de neutrones por escape y absorción y la ganancia neta de neutrones.

La ecuación 5.2 describe esta aproximación, el primer término es la variación del flujo neutrónico en función del tiempo, el segundo es el término de difusión de neutrones, el cual representa el desplazamiento de que los neutrones recorren antes de ser capturados o que escapen del sistema, el 
tercero considera la absorción de los neutrones en función de la sección eficaz macroscópica de absorción y $S(\vec{r}, t)$ es el término fuente.

$$
\frac{1}{v} \frac{\partial \phi(\vec{r}, t)}{\partial t}+\nabla \cdot J(\vec{r}, t)+\Sigma_{a} \phi(\vec{r}, t)=S(\vec{r}, t)
$$

Ahora, para describir la corriente, se utiliza la Ley de Fick (ecuación 5.3), en donde $D$ es el coeficiente de difusión. Se puede observar que aquí se llama Ley de Fick por la similitud con la ley constitutiva de transferencia de masa, no obstante el coeficiente de difusión del neutrón tiene unidades de longitud.

$$
J(\vec{r}, t)=-D(\vec{r}) \nabla \phi(\vec{r}, t)
$$

El coeficiente de difusión del neutrón $D(\vec{r})$ está dado por (Espinosa-Paredes y Vázquez, 2016):

$$
D(\vec{r})=\frac{1}{3 \Sigma_{t r}(\vec{r})}=\frac{1}{3\left[\Sigma_{t}(\vec{r})-\bar{\mu}_{0} \Sigma_{s}(\vec{r})\right]}
$$

Donde $\bar{\mu}_{0}$ es el promedio del coseno del ángulo de dispersión por colisión, dado por $\bar{\mu}_{0}=\frac{3}{2 A}$, con $A$ como el número de masa del moderador. Los términos $\Sigma_{t r}, \Sigma_{t} \mathrm{y} \Sigma_{s}$ son la sección eficaz de transporte, total y de dispersión del material (refrigerante y/o moderador).

Sustituyendo la ecuación (5.3) en la ecuación de difusión se tiene

$$
\frac{1}{v} \frac{\partial \phi(\vec{r}, t)}{\partial t}-D \nabla^{2} \phi(\vec{r}, t)+\Sigma_{a} \phi(\vec{r}, t)=S(\vec{r}, t)
$$

Para el término fuente es necesario considerar los neutrones inmediatos y neutrones retardados que se forman en el proceso de fisión. Los neutrones inmediatos son los que se generan inmediatamente después del proceso de fisión, estos son liberados de manera casi inmediata a partir de los productos de fisión, los neutrones retardados se generan a partir del decaimiento de los productos de fisión, ambos tipos de neutrones contribuyen con el modelo de difusión. Si $\beta$ es la fracción de neutrones retardados entonces $1-\beta$ es la fracción de neutrones instantáneos, lo que hace que el término fuente este dado por la ecuación (5.6)

$$
S(\vec{r}, t)=(1-\beta) k_{\infty} \Sigma_{f} \phi(\vec{r}, t)+\beta k_{\infty} \Sigma_{f} \phi(\vec{r}, t)
$$


El factor de multiplicación infinito $k_{\infty}$ es el cociente de la concentración de neutrones entre dos generaciones sucesivas, en un medio multiplicativo infinito. La sección eficaz para un proceso particular, que se aplica a un solo núcleo, es llamada la sección eficaz microscópica. Y la cantidad de neutrones generados es el flujo de neutrones (Espinosa-Paredes y Vázquez, 2016).

Para los neutrones retardados, la tasa de formación es igual a la tasa de desintegración radioactiva del precursor, en este caso se consideran seis grupos de neutrones retardados, es decir:

$$
\beta k_{\infty} \Sigma_{f} \phi(\vec{r}, t)=\sum_{i=1}^{6} \lambda_{i} \hat{C}_{i}(\vec{r}, t)
$$

donde

$\lambda_{i}=$ constante de desintegración del grupo $i$

$\hat{C}_{i}(\vec{r}, t)=$ concentración de los precursores del grupo $i$

Sustituyendo (5.7) en (5.6) se obtiene:

$$
S(\vec{r}, t)=(1-\beta) k_{\infty} \Sigma_{f} \phi(\vec{r}, t)+\sum_{i=1}^{6} \lambda_{i} \hat{C}_{i}(\vec{r}, t)
$$

Ahora, introduciendo las expresiones para la fuente, la ecuación de difusión queda como:

$$
\frac{1}{v} \frac{\partial \phi(\vec{r}, t)}{\partial t}-D \nabla^{2} \phi(\vec{r}, t)+\Sigma_{f} \phi(\vec{r}, t)=(1-\beta) k_{\infty} \Sigma_{f} \phi(\vec{r}, t)+\sum_{i=1}^{6} \lambda_{i} \hat{C}_{i}(\vec{r}, t)
$$

En la hipótesis de que todos los neutrones tienen la misma energía, la relación entre el coeficiente de difusión $D$ y la sección macroscópica $\Sigma_{f}$ es igual al cuadrado de la longitud de difusión para dicha energía, es decir

$$
\frac{D}{\Sigma_{f}}=L^{2}
$$

En un medio multiplicador, la distribución de flujo neutrónico puede representarse mediante la ecuación (5.11), en donde $B^{2}$ es el buckling del sistema, la cual constituye una medida de la curvatura de la distribución espacial del flujo neutrónico y es inversamente proporcional a las dimensiones del reactor.

$$
\nabla^{2} \phi(\vec{r}, t)+B^{2} \phi(\vec{r}, t)=0
$$


Sustituyendo (5.11) en (5.9) se tiene

$$
\frac{1}{v} \frac{\partial \phi(\vec{r}, t)}{\partial t}+D B^{2} \phi(\vec{r}, t)+\Sigma_{f} \phi(\vec{r}, t)=(1-\beta) k_{\infty} \Sigma_{f} \phi(\vec{r}, t)+\sum_{i=1}^{6} \lambda_{i} \hat{C}_{i}(\vec{r}, t)
$$

Aplicando el método de separación de variables para resolver la ecuación (5.12) se puede considerar que $\phi(\vec{r}, t)$ y $\hat{C}(\vec{r}, t)$ pueden ser representadas como el producto de dos funciones, una dependiente del tiempo y otra de la posición, por lo que se proponen las ecuaciones (5.13a) y (5.13b).

$$
\begin{aligned}
& \phi(\vec{r}, t)=v n(t) \psi(\vec{r}) \\
& \hat{C}(\vec{r}, t)=C(t) \psi(\vec{r})
\end{aligned}
$$

En donde $v$ es la velocidad media de los neutrones, $n(t)$ es la densidad neutrónica en función del tiempo, $C(t)$ es la concentración de neutrones en función del tiempo y $\psi(\vec{r})$ es la función de la posición.

Sustituyendo (5.13a y b) en (5.12) se obtiene

$$
\frac{d n(t)}{d t}=k_{\infty} v \Sigma_{f}\left[(1-\beta)-\frac{D B^{2}}{k_{\infty} \Sigma_{f}}-\frac{1}{k_{\infty}}\right] n(t)+\sum_{i=1}^{6} \lambda_{i} C_{i}(t)
$$

Sustituyendo la ecuación (5.10) en (5.14), la ecuación de difusión se transforma en

$$
\frac{d n(t)}{d t}=\Sigma_{f} v k_{\infty}\left[(1-\beta)-\frac{1+L^{2} B^{2}}{k_{\infty}}\right] n(t)+\sum_{i=1}^{6} \lambda_{i} C_{i}(t)
$$

Una propiedad fundamental en el sistema multiplicador de neutrones es el factor de multiplicación efectivo, $k_{\text {eff }}$, el cual se define como la relación entre el número de neutrones que se producen por fisión en cada generación y número de neutrones absorbidos en la generación anterior, correspondiente a un sistema de tamaño infinito.

Si $k_{\text {eff }}$ es igual a uno, significa que el reactor está en estado crítico, es decir, no existen alteraciones en la población de neutrones de una a otra generación. Si es mayor que uno, la condición del reactor se considera súper crítico, el sistema está produciendo más neutrones que los que son absorbidos. Y si el factor de multiplicación es menor que uno, se tiene una condición subcrítica, en donde el sistema está produciendo menos neutrones de los absorbidos, es decir, se extingue y por tal motivo no puede mantener la reacción de forma sostenida. 
En este caso, el factor de multiplicación efectivo se deduce como

$$
k_{e f f}=k_{\infty} P_{N L}
$$

En donde $P_{N L}$ es la probabilidad de no escape, es decir, es la probabilidad de que no escapen físicamente neutrones del sistema, de que un neutrón no cruce la frontera del reactor como neutrón rápido o térmico. Es la relación entre el número de neutrones absorbidos por segundo en el reactor y los que son producidos en total (Espinosa-Paredes y Vázquez, 2016), éste término está dado por:

$$
P_{N L}=\frac{1}{1+L^{2} B^{2}}
$$

Por lo tanto, el factor de multiplicación efectivo queda:

$$
k_{e f f}=\frac{k_{\infty}}{1+L^{2} B^{2}}
$$

Introduciendo la ecuación (5.18) en la ecuación de difusión, se tiene

$$
\frac{d n(t)}{d t}=\Sigma_{f} v k_{\infty}\left[\left(1-\frac{1}{k_{\text {eff }}}\right)-\beta\right] n(t)+\sum_{i=1}^{6} \lambda_{i} C_{i}(t)
$$

Ahora, para los neutrones inmediatos, la ecuación que define el tiempo de generación de éstos es

$$
\Lambda=\frac{1}{k_{\infty} v \Sigma_{f}}
$$

Este término solo depende de la composición y del tipo de reactor, siendo independiente del tamaño ya que solo depende del factor de multiplicación, la velocidad y la sección eficaz macroscópica.

Otro factor importante en el control de la potencia de un reactor es el concepto de reactividad, $\rho$, definida por la ecuación (5.21). Este término se refiere al cambio fraccional en la población de neutrones por generación.

$$
\rho \equiv \frac{k_{e f f}-1}{k_{e f f}}
$$

Sustituyendo las expresiones de la reactividad y la velocidad de generación de los neutrones inmediatos en la ecuación (5.12) se obtiene 


$$
\frac{d n(t)}{d t}=\left(\frac{\rho-\beta}{\Lambda}\right) n(t)+\sum_{i=1}^{6} \lambda_{i} C_{i}(t)
$$

Para conocer la densidad neutrónica, es necesario conocer la variación de las concentraciones $C_{i}$ de los precursores para los neutrones retardados. Esta concentración está dada por la siguiente expresión

$$
\frac{\partial \hat{C}_{i}(\vec{r}, t)}{\partial t}=\beta_{i} k_{\infty} \Sigma_{f} \phi(\vec{r}, t)-\lambda_{i} \hat{C}_{i}(\vec{r}, t)
$$

En donde $\beta_{i}$ es la fracción del grupo retardado $i$ y $\lambda_{i} C_{i}$ es la velocidad de desintegración simultánea del precursor. Recordando las ecuaciones (5.13a) y (5.13b) realizando el mismo procedimiento que para el flujo neutrónico y sustituyendo (5.20) se tiene

$$
\frac{d C_{i}(t)}{d t}=\frac{\beta_{i}}{\Lambda} n(t)-\lambda_{i} C_{i}(t)
$$

Con este desarrollo se obtienen las ecuaciones que constituyen el sistema de la cinética puntual de reactores, este sistema es el que se utilizara para describir los fenómenos neutrónicos que ocurren dentro del núcleo de un reactor nuclear rápido:

$$
\begin{array}{cc}
\frac{d n(t)}{d t}=\left(\frac{\rho-\beta}{\Lambda}\right) n(t)+\sum_{i=1}^{6} \lambda_{i} C_{i}(t) & n=n_{0} \text { en } t=0 \\
\frac{d C_{i}(t)}{d t}=\frac{\beta_{i}}{\Lambda} n(t)-\lambda_{i} C_{i}(t) & c_{i}=\frac{\beta_{i}}{\lambda_{i} \Lambda} n_{0} \text { en } \quad t=0
\end{array}
$$

\subsubsection{Parámetros neutrónicos}

Los parámetros necesarios para la solución del modelo que se requieren son:

La reactividad $\rho$, está definida por la ecuación (5.25). Ésta cuenta con varias contribuciones: el margen de reactividad inicial $\left(\rho_{0}\right)$, efecto Doppler, expansión del combustible, expansión del encamisado y el efecto de la expansión del plomo (Ponciroli, 2014; Waltar et al., 2012).

$$
\rho=\rho_{0}+\underbrace{1.1 K_{D} \ln \left(\frac{\left\langle T_{\text {fuel }}\right\rangle_{\text {out }}}{\left\langle T_{\text {fuel }}\right\rangle_{\text {in }}}\right)}_{\text {Doppler }}+\underbrace{\alpha_{f} \Delta\left\langle T_{\text {fuel }}\right\rangle}_{\text {Expasión del combustible }}+\underbrace{\alpha_{c} \Delta\left\langle T_{\text {clad }}\right\rangle}_{\text {Expansión del encamisado }}+\underbrace{\alpha_{l} \Delta\left\langle T_{\text {lead }}\right\rangle}_{\text {Expansión del plomo }}
$$


Los parámetros de cada contribución son: constante Doppler $K_{D}=-555 \mathrm{pcm}$, coeficientes de reactividad $\alpha_{f}=-0.232 p c m K^{-1}, \alpha_{c}=0.045 p c m K^{-1}$ y $\alpha_{l}=-0.247 p c m K^{-1}$ (Ponciroli, 2014; Waltar et al., 2012). Es importante notar que $\alpha_{c}$ incluye los efectos de la expansión axial y radial en el encamisado.

Los cambios en la temperatura promedio en la ecuación (5.25), son definidas como:

$$
\begin{aligned}
& \Delta\left\langle T_{\text {fuel }}\right\rangle=\left\langle T_{\text {fuel }}\right\rangle-\left\langle T_{\text {fuel }}\right\rangle_{0} \\
& \Delta\left\langle T_{\text {clad }}\right\rangle=\left\langle T_{\text {clad }}\right\rangle-\left\langle T_{\text {clad }}\right\rangle_{0} \\
& \Delta\left\langle T_{\text {lead }}\right\rangle=\left\langle T_{\text {lead }}\right\rangle-\left\langle T_{\text {lead }}\right\rangle_{0}
\end{aligned}
$$

en donde el subíndice 0 representa la temperatura de referencia. Las temperaturas promedio para calcular la reactividad total están definidas como sigue:

$$
\begin{gathered}
\left\langle T_{\text {fuel }}\right\rangle_{\text {in }}=\left.\frac{1}{L} \int_{z=0}^{z=L} T_{\text {fuel }}\right|_{r=r_{i}} d z \\
\left\langle T_{\text {fuel }}\right\rangle_{\text {out }}=\left.\frac{1}{L} \int_{z=0}^{z=L} T_{\text {fuel }}\right|_{r=r_{f}} d z \\
\left\langle T_{\text {fuel }}\right\rangle=\frac{1}{V_{\text {fuel }}} \int_{V_{\text {fuel }}} T_{\text {fuel }} d V \\
\left\langle T_{\text {clad }}\right\rangle=\frac{1}{V_{\text {clad }}} \int_{V_{\text {clad }}} T_{\text {clad }} d V \\
\left\langle T_{\text {lead }}\right\rangle=\frac{1}{L} \int_{z=0}^{z=L} T_{\text {lead }} d z
\end{gathered}
$$

En donde $r_{i}$ y $r_{f}$ son radios de la región anular interior y de la región del combustible respectivamente. En estas ecuaciones $L$ representa la longitud activa en el núcleo y $V$ es el volumen.

La fracción efectiva de neutrones retardados, $\beta$, está en función de las fracciones efectivas de los neutrones retardados del grupo $i$, estos valores se muestran en la Tabla 5.1. En este trabajo solo se consideró la fracción efectiva del Plutonio-239 debido a que es el material principal del combustible. 


$$
\beta=\sum_{i=1}^{6} \beta_{i}
$$

La constante de decaimiento $\lambda_{i}$ se considera para cada grupo de los precursores, los valores para este caso se encuentran también en la Tabla 5.1.

Tabla 5.1. Parámetros de los neutrones retardados para el Plutonio-239 (Waltar et al., 2012)

\begin{tabular}{|c|c|c|c|c|}
\hline $\begin{array}{c}\text { Total de neutrones } \\
\text { por fisión }\end{array}$ & $\beta$ & Grupo & $\lambda_{i}\left(\mathrm{~s}^{-1}\right)$ & $\beta_{i} / \beta$ \\
\hline \multirow{6}{*}{2.93} & \multirow{6}{*}{0.00215} & 1 & 0.0129 & 0.038 \\
\hline & & 2 & 0.0311 & 0.280 \\
\hline & & 3 & 0.134 & 0.216 \\
\hline & & 4 & 0.331 & 0.328 \\
\hline & & 5 & 1.26 & 0.103 \\
\hline & & 6 & 3.21 & 0.035 \\
\hline
\end{tabular}

El tiempo de generación es $\Lambda=6.116 \times 10^{-7} s$ (Ponciroli, 2014).

\subsubsection{Método de solución numérica}

La solución numérica de la potencia neutrónica considera dos métodos. El primero es el método RungeKutta de cuarto orden, y se aplica para la solución numérica de la densidad de neutrones.

Aplicando el método se tiene

$$
n^{t+\Delta t}=n^{t}+\frac{\Delta t}{6}\left(m_{1}+2 m_{2}+2 m_{3}+m_{4}\right)
$$

En donde $n$ es la densidad neutrónica y $\Delta t$ es el paso de integración, en este caso se utilizó $0.0001 \mathrm{~s}, \mathrm{y}$ cada término $m$ está dado por

$$
m_{1}=\frac{d n}{d t}(n), \quad m_{2}=\frac{d n}{d t}\left(n+\frac{m_{1} \Delta t}{2}\right), \quad m_{3}=\frac{d n}{d t}\left(n+\frac{m_{2} \Delta t}{2}\right), \quad m_{4}=\frac{d n}{d t}\left(n+m_{3} \Delta t\right)
$$

Para $i=1,2, \ldots, 6$ 
El segundo método es Euler, y se aplica para la solución numérica de la concentración del i-ésimo precursor retardado de neutrones, dado por la Ec. 5.37. La longitud de paso utilizada en este trabajo fue de $0.0001 \mathrm{~s}$.

$$
C_{i}^{t+\Delta t}=C_{i}^{t}+\Delta t \frac{d C_{i}}{d t}
$$

\subsection{Análisis de transferencia de calor en la barra de combustible}

El transporte de energía se lleva a cabo mediante diversos mecanismos: convección, conducción y radiación térmica (Bird et al., 2014).

La convección es la transmisión de calor por movimiento de las moléculas de una sustancia. Este fenómeno sólo podrá producirse en fluidos en los que por movimiento natural (diferencia de densidades) o circulación forzada (con la ayuda de ventiladores, bombas, etc.) puedan las partículas desplazarse transportando el calor sin interrumpir la continuidad física del cuerpo.

La conducción es el fenómeno consistente en la propagación de calor entre dos cuerpos o partes de un mismo cuerpo a diferente temperatura debido a la agitación térmica o vibración de moléculas o átomos.

La radiación térmica es la transmisión de calor debido a la potencia emisiva entre cuerpos, los cuales en un instante dado, tienen temperaturas distintas, sin que entre ellos exista contacto ni conexión por otro sólido conductor.

\subsubsection{Modelo de transferencia de calor}

El proceso de transferencia de calor dentro de la barra de combustible es uno de los procesos más importantes dentro del núcleo del reactor. La transferencia de calor analizada es en el perfil radial, considerando cuatro zonas; la zona I es la perforación dentro del pin de combustible, en el cual se encuentra un gas y no es una fuente de calor, la zona II el combustible y es en donde se tiene la fuente, el gap corresponde a la zona III el cual también es gas y la zona $I V$ es el encamisado, en la sección 4.1 se describen las características de cada una de estas zonas. En la Figura 5.2 se muestran los radios para cada una de las áreas a estudiar, $r_{i}$ es el radio de la perforación, $r_{f}$ es el radio del combustible, $r_{g}$ el radio del gap y $r_{c l}$ el radio del encamisado. 
$\mathrm{Al}$ tener un elemento en el cual no existe un desplazamiento de los materiales por los cuales se transfiere la energía, se considera que el fenómeno de transferencia de energía predominante es la conducción. También se considera convección debido a la interacción de los fluidos (helio y plomo), sin embargo la radiación será considerada en futuras aproximaciones del modelo.

Para la mayoría de los problemas ingenieriles la ecuación de energía calorífica resulta conveniente para operar en transmisión de calor (Bird et al., 2014).

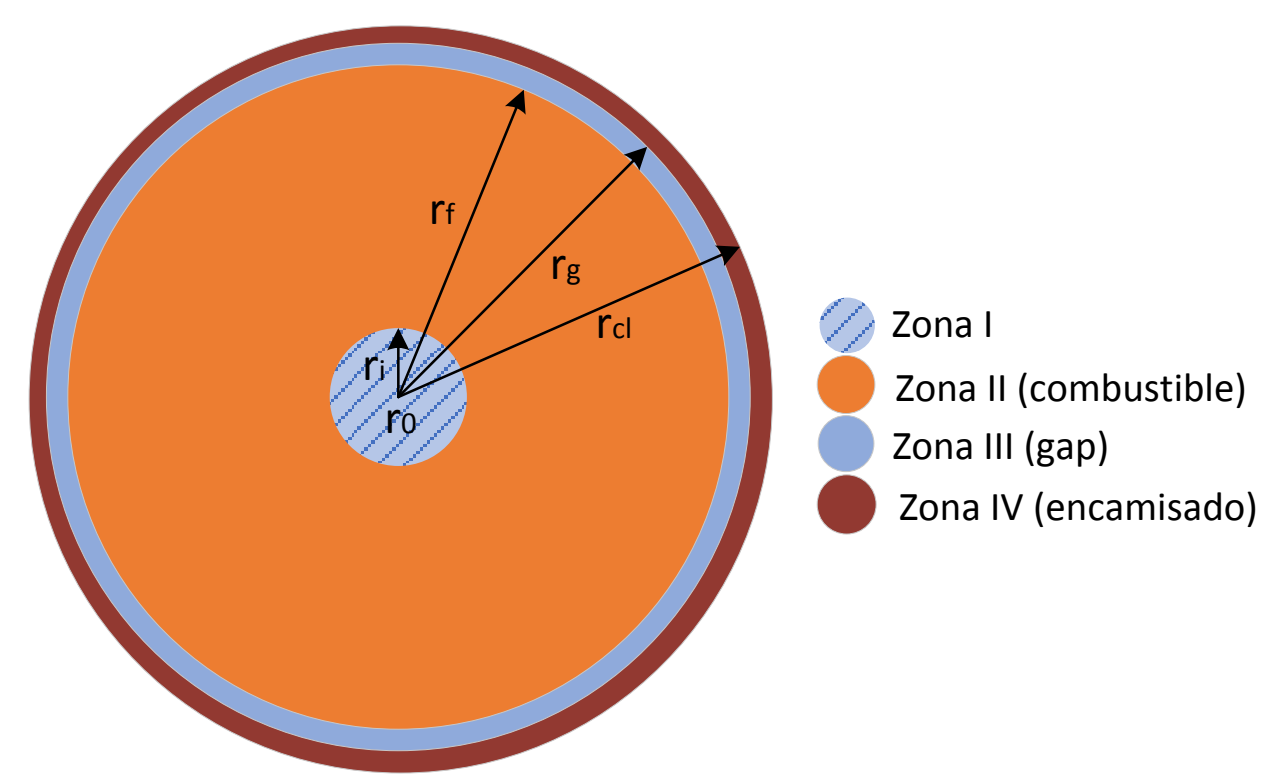

Figura. 5.2. Radios de cada sección en la barra de combustible.

$$
\rho \frac{D \hat{U}}{D t}=-(\nabla \cdot q)-p(\nabla \cdot v)-(\tau: \nabla v)
$$

En donde en primer término se refiere a la tasa de ganancia de energía interna por unidad de volumen, donde $\rho$ es la densidad, $\hat{U}$ es la energía interna y $t$ es el tiempo. El segundo término corresponde a la tasa de entrada de energía interna por unidad de volumen debido a la conducción en donde $q$ es el flujo de calor por unidad de área. El tercer término es la tasa reversible de aumento de energía interna por unidad de volumen debido a la compresión, en este término, $p$ es la presión y $v$ es la velocidad. Y finalmente el cuarto término se refiere a la tasa irreversible de aumento de energía interna por unidad de volumen debido a la disipación viscosa. 
Para este caso es necesario escribir la ecuación en función de la temperatura y del calor específico del fluido en vez de la energía interna, de forma que nos queda la ecuación de energía (5.39), ésta permite calcular los perfiles de temperatura, establece que la temperatura varía debido a: (a) conducción de calor, (b) efectos de expansión, y (c) calentamiento viscoso.

$$
\rho C_{v} \frac{D T}{D t}=k \nabla^{2} T-T\left(\frac{\partial p}{\partial T}\right)_{p}(\nabla \cdot v)+\mu \Phi_{v}
$$

Se puede observar que el operador usado en (5.38) y (5.39) es

$$
\frac{D}{D t}=\frac{\partial}{\partial t}+\vec{v} \cdot \nabla
$$

Para el caso de conducción en sólidos, se omite el término de disipación viscosa $\Phi_{v}$ y la velocidad $v=0$, quedando la ecuación de forma:

$$
\rho C_{v} \frac{\partial T}{\partial t}=k \nabla^{2} T
$$

Con la ecuación (5.41) se puede describir la sección que corresponde al combustible y al encamisado.

Para la sección de la perforación y el gap consideramos que se tiene un fluido a presión constante, de igual forma, despreciamos la disipación viscosa y tenemos la ecuación (5.42)

$$
\rho C_{v} \frac{D T}{D t}=k \nabla^{2} T
$$

Pero también se considera que la velocidad es cero, por lo tanto no se considera la derivada sustancial y la ecuación queda de la misma forma que (5.41).

La formulación de la transferencia de calor en el combustible está basada en las siguientes suposiciones:

- Transferencia de calor radial simétrica: $\frac{\partial T}{\partial r}=0$.

- La conducción de calor en la dirección axial es despreciable con respecto la conducción de calor en la dirección radial: $q_{\theta}, q_{z}<<q_{r}$.

- La tasa de generación de calor volumétrico en el combustible es uniforme en la dirección radial.

- El espacio del gap es considerado uniforme. 
Bajo estas suposiciones, las ecuaciones que describen la distribución de temperatura en cada región son (Figura 5.1):

$$
\begin{gathered}
\rho_{\text {fuel }} C p_{\text {fuel }} \frac{\partial T_{\text {fuel }}}{\partial t}=\frac{k_{\text {fuel }}}{r} \frac{\partial}{\partial r}\left(r \frac{\partial T_{\text {fuel }}}{\partial r}\right)+q^{\prime \prime \prime}(t), \quad \text { Zona I y Zona II } \quad r_{0}<r \leq r_{\text {fuel }} \\
\rho_{\text {gap }} C p_{\text {gap }} \frac{\partial T_{\text {gap }}}{\partial t}=\frac{k_{\text {gap }}}{r} \frac{\partial}{\partial r}\left(r \frac{\partial T_{\text {gap }}}{\partial r}\right), \\
\rho_{\text {clad }} C p_{\text {clad }} \frac{\partial T_{\text {clad }}}{\partial t}=\frac{k_{\text {clad }}}{r} \frac{\partial}{\partial r}\left(r \frac{\partial T_{\text {clad }}}{\partial r}\right), \quad \text { Zona III } r_{\text {fuel }}<r \leq r_{\text {gap }}
\end{gathered}
$$

En el caso de las zonas I y II se considera la misma ecuación para describir la transferencia de calor, a reserva de que en la zona I $q^{\prime \prime \prime}=0$.

La condición inicial está dada por

$$
T(r, 0)=f(r)
$$

Y las condiciones de frontera son:

$$
\begin{array}{ll}
\frac{d T_{\text {fuel }}}{d r}=0 \quad \text { en } r=r_{0} & \\
-k_{\text {gap }} \frac{d T_{\text {gap }}}{d r}=h_{\text {gap }}\left(T_{\text {fuel }}-T_{\text {gap }}\right) & \text { en } r=r_{\text {fuel }} \\
-k_{\text {clad }} \frac{d T_{\text {clad }}}{d r}=h_{\text {gap }}\left(T_{\text {gap }}-T_{\text {clad }}\right) & \text { en } r=r_{\text {gap }} \\
k_{\text {clad }} \frac{d T_{\text {clad }}}{d r}=h_{\text {lead }}\left(T_{\text {clad }}-T_{\text {lead }}\right) & \text { en } r=r_{\text {clad }}
\end{array}
$$

En estas ecuaciones $\rho$ es la densidad, $C p$ es el calor específico, $k$ es la conductividad térmica, $h$ es el coeficiente de transferencia de calor por convección y $q^{\prime \prime \prime}$ es la fuente de calor. El subíndice fuel se refiere al combustible, gap se refiere al gap o huelgo, clad al clad y lead se refiere al refrigerante.

El término fuente está dado por: 


$$
q^{\prime \prime \prime}(t, z)=\frac{P(t, z)}{V_{f}}
$$

Donde $P(t)$ es la potencia del reactor definida en la ecuación (5.1) y $V_{f}$ es el volumen del combustible.

\subsubsection{Parámetros de transferencia de calor}

El modelo de transferencia de calor en el combustible descrito anteriormente requiere de varios parámetros que ayuden a describir el fenómeno en el combustible, el gap y el encamisado.

Las correlaciones que describen la densidad, $\rho$, la capacidad calorífica, $C p$, y la conductividad térmica $k$, para el MOX de combustible se presentan en la sección 4.1. En esa misma sección se muestran las características del gap (helio) y el encamisado (T91).

\subsubsection{Método de solución}

Aplicando el método de diferencias finitas en forma implícita para las ecuaciones (5.43-45), en donde

$$
\rho_{i} C_{p_{i}} \frac{\partial T}{\partial t} \approx \rho_{i} C_{p_{i}}\left[\frac{T_{i}^{t+\Delta t}-T_{i}^{t}}{\Delta t}\right]
$$

para el lado derecho de la ecuación se tiene

$$
\frac{k_{i}}{r_{i}} \frac{\partial}{\partial r}\left(r_{i} \frac{\partial T}{\partial r}\right) \approx k_{i} \frac{T_{i+1}^{t+\Delta t}-2 T_{i}^{t+\Delta t}+T_{i-1}^{t+\Delta t}}{\Delta r^{2}}+\frac{k_{i}}{r_{i} \Delta r}\left[T_{i+1}^{t+\Delta t}-T_{i}^{t+\Delta t}\right]
$$

Sustituyendo

$$
\rho_{i} C p_{i}\left[\frac{T_{i}^{t+\Delta t}-T_{i}^{t}}{\Delta t}\right]=k_{i}\left[\frac{T_{i+1}^{t+\Delta t}-2 T_{i}^{t+\Delta t}+T_{i-1}^{t+\Delta t}}{\Delta r^{2}}\right]+\frac{k_{i}}{r_{i} \Delta r}\left[T_{i+1}^{t+\Delta t}-T_{i}^{t+\Delta t}\right]+q_{i}^{\prime \prime \prime}
$$

Reescrita la ecuación (5.54) de forma algebraica se obtiene

$$
a_{i} T_{i-1}^{t+\Delta t}+b_{i} T_{i}^{t+\Delta t}+c_{i} T_{i+1}^{t+\Delta t}=d_{i}, \quad \text { en } \quad i=2,3,4 \ldots n-1
$$

Donde 
$a_{i}=\frac{k_{i}}{\Delta r^{2}}$

$b_{i}=-\frac{2 k_{i}}{\Delta r^{2}}-\frac{k_{i}}{r_{i} \Delta r}-\frac{\rho_{i} C p_{i}}{\Delta r}$

$c_{i}=\frac{k_{i}}{\Delta r^{2}}+\frac{k_{i}}{r_{i} \Delta r}$

$d_{i}=-\frac{\rho_{i} C p_{i}}{\Delta r}-q_{i}^{\prime \prime \prime}, \quad$ en $\quad i=2,3 \ldots, 9 \quad$ (zona de combustible)

$d_{i}=-\frac{\rho_{i} C p_{i}}{\Delta r}, \quad$ en $\quad i=10,11 \ldots, 18 \quad$ (zona de gap y encamisado)

Las condiciones de frontera mostradas en las ecuaciones (5.47-50) en forma discretizada resultan en:

$$
\begin{gathered}
T_{1}^{t+\Delta t}=T_{2}^{t+\Delta t}, \quad \text { en } \quad r=r_{0} \\
T_{n}^{t+\Delta t}\left(\frac{-k_{n}}{\Delta r}-h_{\text {lead }}\right)+T_{n-1}^{t+\Delta t}\left(\frac{k_{n}}{\Delta r}\right)=-h_{\text {lead }} T_{\text {lead }}, \text { para } \quad r=r_{c l}
\end{gathered}
$$

La ecuación (5.55) tiene una estructura matricial de tipo tridiagonal, que se resuelve con el algoritmo de Thomas.

\subsection{Análisis del termofluido}

En este apartado se desarrollan los modelos para describir el refrigerante, realizando balances de materia, cantidad de movimiento y energía a nivel macroscópico. El sistema consiste en una barra de combustible y el refrigerante que la rodea.

En la Figura 5.3 se observa el sistema a modelar. Se encuentra en coordenadas cilíndricas, la sección amarilla es la barra de combustible, de donde se tiene flujo de calor hacia el refrigerante (sección azul), como parámetros de entrada al sistema tenemos flujo de refrigerante el cual lleva una velocidad y temperatura determinadas, pero al pasar por la barra, el refrigerante extrae el calor y la temperatura de salida es diferente. 


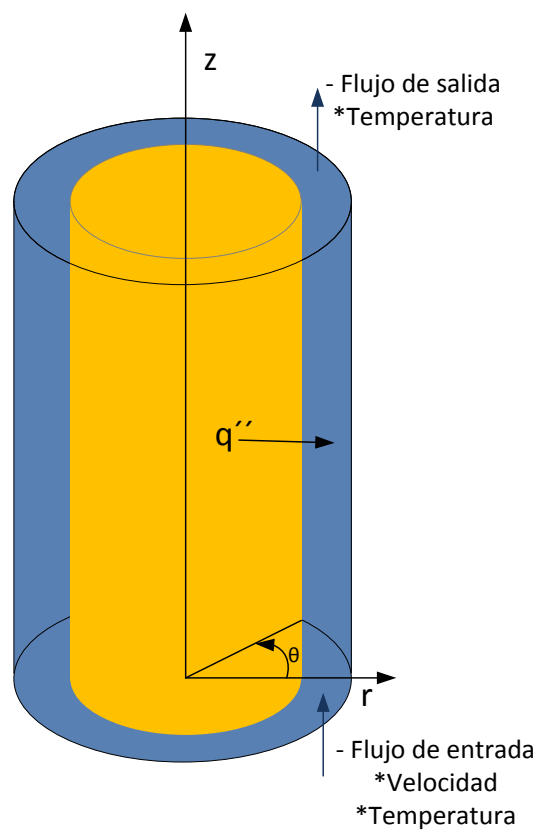

Figura 5.3. Sistema para el análisis termofluido.

\subsubsection{Balance de masa}

Una ecuación de balance nos permite considerar las entradas, salidas, tasa de generación y de consumo para determinar la tasa de acumulación de un sistema, estos balances pueden ser aplicados en masa, cantidad de movimiento o energía.

Para iniciar consideraremos la ecuación de continuidad (5.58) la cual es una expresión matemática para expresar que la masa se conserva, describe la variación de la densidad para un punto fijo, como consecuencia de las variaciones del vector velocidad másica (Bird et al.,2014).

$$
\frac{\partial \rho}{\partial t}+\nabla \cdot(\rho \mathbf{v})=0
$$

Ahora, definiendo el promedio de una propiedad como la ecuación (5.59) (Whitaker, 1983)

$$
\langle\bullet\rangle=\frac{1}{V} \int_{V}^{\bullet} d V
$$

Con esta expresión se estima el promedio de las velocidades, de forma que es posible obtener balances macroscópicos. Ahora aplicando la ecuación (5.59) a (5.58) y desarrollando el gradiente se tiene 


$$
\left\langle\frac{\partial \rho}{\partial t}\right\rangle+\left\langle\frac{1}{r} \frac{\partial}{\partial r}\left(\rho r v_{r}\right)+\frac{1}{r} \frac{\partial}{\partial \theta}\left(\rho v_{\theta}\right)+\frac{\partial}{\partial z}\left(\rho v_{z}\right)\right\rangle=0
$$

En este caso podemos despreciar el cambio de la velocidad en las coordenadas $r, \theta$ ya que el fluido se desplaza en promedio solo en dirección $z$. Ahora la ecuación de continuidad queda

$$
\frac{\partial \rho}{\partial t}+\frac{\partial \rho\left\langle v_{z}\right\rangle}{\partial z}=0
$$

En el este caso, al desarrollar el análisis sobre un metal líquido existe un término que debemos considerar, el coeficiente de expansión en volumen, denotado por $\alpha$. Este coeficiente establece la relación entre el cambio en el volumen y la temperatura a una presión constante y se define mediante la ecuación (Bird et al., 2014)

$$
\alpha=\frac{1}{V}\left(\frac{\partial V}{\partial T}\right)_{p}=\frac{1}{(1 / \rho)}\left(\frac{\partial(1 / \rho)}{\partial T}\right)_{p}=-\frac{1}{\rho}\left(\frac{\partial \rho}{\partial T}\right)_{p}
$$

Ahora, introduciendo el coeficiente de expansión en la ecuación (5.61) se tiene

$$
-\alpha \rho \frac{\partial T}{\partial t}+\frac{\partial \rho\left\langle v_{z}\right\rangle}{\partial z}=0
$$

donde

$$
\frac{\partial \rho}{\partial t}=-\alpha \rho \frac{\partial T_{l e a d}}{\partial t}
$$

Definiendo $G$ y sustituyéndolo en (5.63)

$$
\begin{gathered}
G=\rho\langle v\rangle \\
\alpha \rho \frac{\partial T_{\text {lead }}}{\partial t}-\frac{\partial G}{\partial z}=0
\end{gathered}
$$

Finalmente la expresión (5.65) es la ecuación de continuidad que describe el sistema. 


\subsubsection{Balance de cantidad de movimiento}

Para esta sección partimos de la ecuación de movimiento (Bird et al., 2014) (5.66). Esta ecuación describe la razón de cambio en el tiempo de la cantidad de movimiento en un fluido y las fuerzas que actúan sobre él.

$$
\frac{\partial \rho \mathbf{v}}{\partial t}+\nabla \cdot \rho \mathbf{v v}+\nabla \cdot \mathbf{p}+\nabla \cdot \boldsymbol{\tau}-\rho \mathbf{g}=0
$$

Aplicando la ecuación (5.59) a cada término de la ecuación (5.66) para obtener los valores promedios se obtiene

$$
\left\langle\frac{\partial \rho \mathbf{v}}{\partial t}\right\rangle=\frac{\partial}{\partial t}\langle\rho \mathbf{v}\rangle=\frac{\partial}{\partial t} \rho\langle\mathbf{v}\rangle
$$

Para el segundo término aplicamos el teorema de la divergencia, en este caso el valor de la integral es cero, ya que $v$ y $n$ son vectores ortogonales y su producto punto es igual a cero.

$$
\langle\nabla \cdot \rho \mathbf{v v}\rangle=\nabla \cdot\langle\rho \mathbf{v v}\rangle+\frac{1}{V} \int_{A} \rho(\mathbf{v v})(v \cdot n) d A
$$

El vector velocidad es la suma del promedio de la velocidad y la desviación de la velocidad, es decir

$$
\mathbf{v}=\langle\mathbf{v}\rangle+\langle\tilde{\mathbf{v}}\rangle
$$

Aplicando esta definición a (5.68) se tiene

$$
\langle\nabla \cdot \rho \mathbf{v v}\rangle=\nabla \cdot \rho\langle\mathbf{v}\rangle\langle\mathbf{v}\rangle+\nabla \cdot \rho\langle\tilde{\mathbf{v}} \tilde{\mathbf{v}}\rangle
$$

Continuando para el término de la presión y de los esfuerzos cortantes

$$
\begin{aligned}
& \langle\nabla \cdot \mathbf{p}\rangle=\frac{1}{V} \int_{V} \nabla \cdot \mathbf{p} d V=\nabla \cdot\langle\mathbf{p}\rangle \\
& \langle\nabla \cdot \boldsymbol{\tau}\rangle=\frac{1}{V} \int_{V} \nabla \cdot \boldsymbol{\tau} d V=\nabla \cdot\langle\boldsymbol{\tau}\rangle
\end{aligned}
$$

Sustituyendo las ecuaciones (5.67-72) se tiene 


$$
\frac{\partial}{\partial t} \rho\langle\mathbf{v}\rangle+\nabla \cdot \rho\langle\mathbf{v}\rangle\langle\mathbf{v}\rangle+\nabla \cdot \rho\langle\tilde{\mathbf{v}} \tilde{\mathbf{v}}\rangle+\nabla \cdot\langle\mathbf{p}\rangle+\nabla \cdot\langle\boldsymbol{\tau}\rangle-\rho \mathbf{g}=0
$$

Como se comentó en la sección anterior, se pueden despreciar los efectos en dirección $r$ y $\theta$ por lo tanto proyectamos en dirección a $z$

$$
\frac{\partial \rho\left\langle\mathbf{v}_{z}\right\rangle}{\partial t}+\frac{\partial \rho\left\langle\mathbf{v}_{z}\right\rangle\left\langle\mathbf{v}_{z}\right\rangle}{\partial z}+\nabla \cdot \rho\langle\tilde{\mathbf{v}} \tilde{\mathbf{v}}\rangle+\frac{\partial\langle\mathbf{p}\rangle}{\partial z}+\nabla \cdot\langle\boldsymbol{\tau}\rangle-\rho g_{z}=0
$$

Ahora bien, recordando que $G=\rho\langle v\rangle$ la ecuación de cantidad de movimiento queda

$$
\frac{\partial G}{\partial t}+\frac{1}{\rho} \frac{\partial G^{2}}{\partial z}+\nabla \cdot \rho\langle\tilde{\mathbf{v}} \tilde{\mathbf{v}}\rangle+\frac{\partial\langle\mathbf{p}\rangle}{\partial z}+\nabla \cdot\langle\boldsymbol{\tau}\rangle-\rho g_{z}=0
$$

A demás el coeficiente de fricción se define como

$$
\xi_{f r}=\frac{0.210}{\operatorname{Re}^{0.25}} \frac{L}{D_{h}}\left[1+\left(\frac{l_{p}}{D_{\text {rod }}}-1\right)^{0.32}\right]
$$

Donde $L$ es la longitud de la barra, $D_{h}$ es el diámetro hidráulico, $l_{p}$ es el pitch (longitud entre cada barra) y $D_{\text {rod }}$ es el diámetro de la barra.

Por lo tanto a ecuación de balance de cantidad de movimiento es

$$
\frac{\partial G}{\partial t}=-\frac{\xi_{f r}}{2}\left(\frac{G^{2}}{\rho_{\text {lead }} L}\right)-\frac{\partial}{\partial z}\left(\frac{G^{2}}{\rho_{\text {lead }}}\right)-\rho_{\text {lead }} g
$$

\subsubsection{Balance de energía}

Como en los casos anteriores el punto de partida es la ecuación en forma local, la cual se promedia para obtener:

$$
\frac{\partial \rho h}{\partial t}+\frac{\partial \rho\langle v\rangle h}{\partial z}=\frac{q^{\prime \prime} P_{c}}{A_{f}}
$$

donde $P_{c}$ es el perímetro calentado y $A_{f}$ es el área de flujo. Expresando en términos del flujo másico por unidad de área: 


$$
\frac{\partial \rho h}{\partial t}+\frac{\partial \rho G}{\partial z}=\frac{q^{\prime \prime} P}{A_{f}}
$$

El interés del modelo es poder predecir la temperatura, por lo que es necesario tener las ecuaciones en función de la temperatura, para el primer término hacemos el siguiente desarrollo

$$
\frac{\partial \rho h}{\partial t}=h \frac{\partial \rho}{\partial t}+\rho \frac{\partial h}{\partial t}
$$

Como $\rho=\rho(T)$, el segundo término del lado derecho está dado por:

$$
\rho \frac{\partial h}{\partial t}=\left(h \frac{\partial \rho}{\partial T}+\rho \frac{\partial h}{\partial T}\right) \frac{\partial T_{\text {lead }}}{\partial t}
$$

Recordando que $-\alpha \rho=\frac{\partial \rho}{\partial T}$ y que la capacidad calorífica está definida como $C_{p}=\frac{\partial h}{\partial T}$, se puede reescribir como

$$
\frac{\partial \rho h}{\partial t}=\frac{\partial T_{\text {lead }}}{\partial t}[C p-\alpha h] \rho
$$

Para el segundo término se tiene utiliza nuevamente la definición del calor específico y seguir el mismo procedimiento, de forma que se obtiene

$$
\left\langle v_{z}\right\rangle \frac{\partial \rho h}{\partial z}=\left\langle v_{z}\right\rangle \rho \frac{\partial T_{l e a d}}{\partial z}
$$

Sustituyendo las ecuaciones (5.80 y 5.81) en (5.78) se obtiene la ecuación de energía en función de la temperatura.

$$
\frac{\partial T_{\text {lead }}}{\partial t}[C p-\alpha h] \rho+\left\langle v_{z}\right\rangle \rho C_{p} \frac{\partial T_{\text {lead }}}{\partial z}=0
$$

Utilizando nuevamente la definición de $G=\rho\langle v\rangle$ y considerando el flujo de calor de la barra de combustible al fluido se tiene que

$$
\frac{\partial T_{\text {lead }}}{\partial t}\left[C_{p}-\alpha h\right] \rho+G C_{p} \frac{\partial T_{\text {lead }}}{\partial z}=\frac{P_{m} q^{\prime \prime}}{A_{f}}
$$

Definiendo el flujo de calor como 


$$
q^{\prime \prime}=h_{\text {lead }}\left(T_{\text {clad }}-T_{\text {lead }}\right)
$$

Donde $h_{\text {lead }}$ es el coeficiente convectivo del plomo y $T_{\text {clad }}, T_{\text {lead }}$ son las temperaturas del encamisado y del plomo respectivamente. Sustituyendo (5.84) en (5.83) y despejando $\frac{\partial T_{\text {lead }}}{\partial t}$ se tiene la ecuación de balance de energía

$$
\frac{\partial T_{\text {lead }}}{\partial t}=\frac{P_{m} h_{\text {lead }}\left(T_{\text {clad }}-T_{\text {lead }}\right)}{A_{f} \rho_{\text {lead }} C p_{\text {lead }}}-\frac{G}{\rho_{\text {lead }}} \frac{\partial T_{\text {lead }}}{\partial z}
$$

A continuación se presenta el modelo del termofluido con las condiciones de iniciales y de frontera, para el balance de materia (5.65) y el de cantidad de movimiento (5.75) son

$$
\alpha \rho \frac{\partial T_{\text {lead }}}{\partial t}-\frac{\partial G}{\partial z}=0 \quad \frac{\partial G}{\partial t}=-\frac{\xi_{f r}}{2}\left(\frac{G^{2}}{\rho_{\text {lead }} L}\right)-\frac{\partial}{\partial z}\left(\frac{G^{2}}{\rho_{\text {lead }}}\right)-\rho_{\text {lead }} g
$$

Condición inicial:

$$
G=G(z)=G_{e n} \text { en } t=0
$$

Condición de frontera:

$$
G=G_{\text {en }} \text { en } \quad z=0
$$

Para el balance de energía (5.85) se tienen

$$
\frac{\partial T_{\text {lead }}}{\partial t}=\frac{P_{m} h_{\text {lead }}\left(T_{\text {clad }}-T_{\text {lead }}\right)}{A_{f} \rho_{\text {lead }} C p_{\text {lead }}}-\frac{G}{\rho_{\text {lead }}} \frac{\partial T_{\text {lead }}}{\partial z}
$$

Condición inicial:

$$
T=T(z) \text { en } t=0
$$

Condición de frontera:

$$
T=T_{\text {en }} \text { en } z=0
$$




\subsubsection{Parámetros}

El análisis se realizara a un canal promedio, éste es la cantidad de flujo de refrigerante promedio por barra de combustible. A continuación se describe la estimación del canal promedio.

Como se mencionó en la sección 3.2, el ensamble de combustible tiene una geometría hexagonal, entonces para calcular el canal promedio tenemos que considerar el flujo que rodea una barra de refrigerante. En la Figura 5.4 se muestra una barra de combustible dentro de un hexágono formado por otras barras de combustible, si se trazan líneas del centro de la barra central hacia cada barra externa el círculo se divide en seis partes, y cada una de estas partes tiene el flujo que pasa, entonces necesitamos considerar todas esas partes para tener el canal promedio.

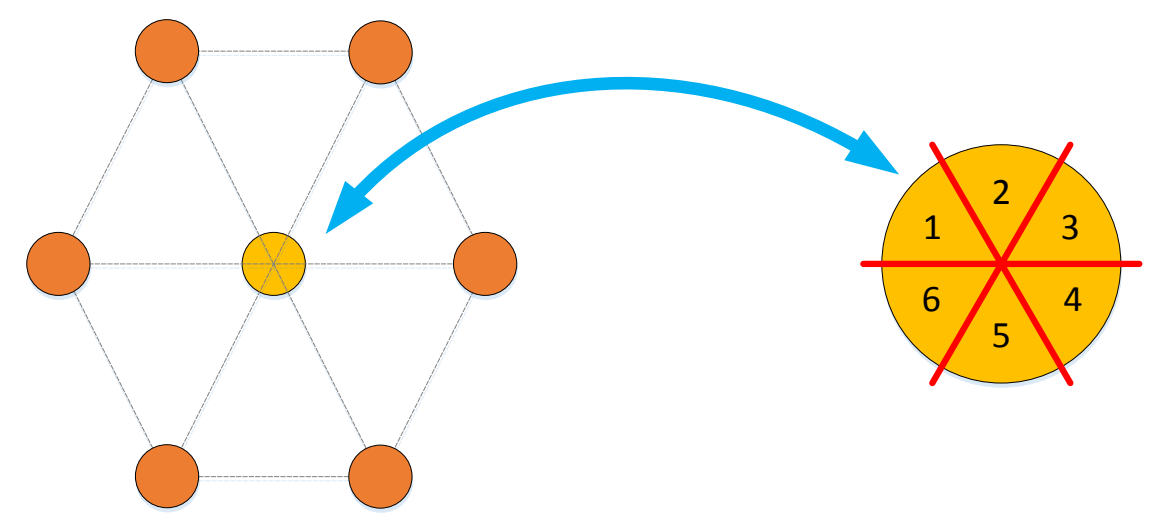

Figura 5.4. Barra de combustible en arreglo hexagonal.

En la Figura 5.5 se muestra que se tienen que considerar dos triángulos para poder calcular el canal promedio y de esta forma tener el flujo de cada barra.

Las dimensiones de las barras de combustible y del ensamble se encuentran en la sección 3.2 del presente trabajo, con estos valores y las ecuaciones descritas se tienen los elementos suficientes para continuar con el modelado. En la Tabla 5.2 se muestran las relaciones para determinar el área de flujo, $A_{f}$, el diámetro hidráulico, $D_{h}$, y el perímetro mojado $P_{m}$. El radio del combustible se denomina $R_{\text {rod }}$ y la longitud entre barra (pitch) es $l_{p}$.

Las propiedades del plomo han sido descritas en la sección 3.4, ahí se encuentra las correlaciones para la densidad, calor específico, conductividad y entalpía. Además de los siguientes números adimensionales de transferencia de calor por barra (Tabla 5.3). 

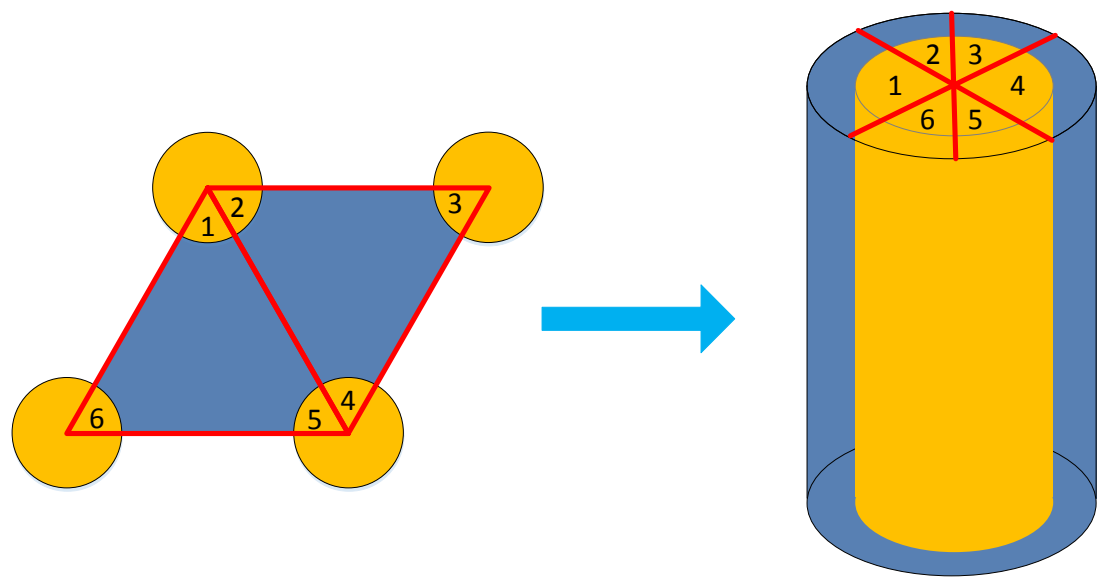

Figura 5.5. Canal promedio para el análisis termofluido.

Tabla 5.2. Parámetros termofluido por canal promedio.

\begin{tabular}{ccc}
\hline Parámetro & Ecuación & Unidades \\
\hline$A_{f}$ & $\frac{\sqrt{3}}{2} l_{p}^{2}-\pi R_{r o d}^{2}$ & $m^{2}$ \\
\hline$D_{h}$ & $\frac{4}{\pi D_{\text {rod }}} A_{f}$ & $m$ \\
\hline$P_{m}$ & $D_{\text {rod }}(\pi-4)+4 l_{p}$ & $m$ \\
\hline
\end{tabular}

Tabla 5.3. Números adimensionales para la transferencia de calor al refrigerante.

\begin{tabular}{cc}
\hline Número & Correlación \\
\hline $\operatorname{Re}$ & $\frac{G D_{f}}{\mu_{\text {lead }}}$ \\
\hline $\mathrm{Nu}$ & $4+0.025(\mathrm{Pr} \mathrm{Re})^{0.8}$ \\
\hline $\mathrm{Pr}$ & $\frac{C p_{\text {lead }} \mu_{\text {lead }}}{k_{\text {lead }}}$
\end{tabular}

\subsubsection{Método de solución}

Para la ecuación 5.85, se desarrolla en aproximación finita para el término espacial alrededor de $s w+1$, resulta la ecuación 


$$
\left.\frac{\partial T}{\partial t}\right|_{s w+1}=\frac{P_{m} h_{\text {lead }}\left(T_{\text {clad }}-T_{s w}\right)}{A_{f} \rho_{l e a d} C p_{l e a d}}-\frac{G_{s w}}{\rho_{l e a d}} \frac{\left(T_{s w+1}-T_{s w}\right)}{\Delta z}
$$

Resolviendo de forma analítica con la dependencia temporal para $s w=1,2,3, \ldots, 24$, resulta

$$
T_{s w+1}=\left(\frac{\omega T_{\text {clad }}+\frac{G}{\rho} T_{s w}}{\omega+\frac{G}{\rho}}\right)\left(1-e^{-\left(\omega+\frac{G}{\rho}\right) t}\right)+T_{o} e^{-\left(\omega+\frac{G}{\rho}\right) t}
$$

donde,

$$
\omega=\frac{h_{\text {lead }} P_{m} \Delta z}{A_{f} \rho_{\text {lead }} C p_{\text {lead }}}
$$

Para la solución numérica de la ecuación 5.75 se emplea una discretización explicita, quedando así para $s w=1,2,3, \ldots, 24$,

$$
\frac{G_{s w}^{t+\Delta t}-G_{s w}^{t}}{\Delta t}=-\frac{\xi_{f r}}{2}\left(\frac{G_{s w}^{2}}{\rho_{\text {lead }} \Delta z}\right)-\frac{\left.\left(G^{2} / \rho_{\text {lead }}\right)\right|_{s w+1} ^{t}-\left.\left(G^{2} / \rho_{\text {lead }}\right)\right|_{s w} ^{t}}{\Delta z}-\rho_{\text {lead }} g
$$

Por lo que la caída de presión total es,

$$
\Delta P_{T}=\sum_{s w=1}^{23}\left(-\frac{\xi_{f r}}{2}\left(\frac{G_{\text {sw }}^{2}}{\rho_{\text {lead }}}\right)-\left[\left.\left(G^{2} / \rho_{\text {lead }}\right)\right|_{\text {sw+1 }} ^{t}-\left.\left(G^{2} / \rho_{\text {lead }}\right)\right|_{s w} ^{t}\right]-\rho_{\text {lead }} g \Delta z\right)
$$

Para la solución numérica de la ecuación 5.65 se aplica el método de Euler,

$$
G_{s w+1}=G_{s w}-\Delta z \alpha \rho_{l e a d}\left(\frac{\partial T_{l e a d}}{\partial t}\right)_{s w}
$$

Con los modelos desarrollados es las secciones 5.1, 5.2, 5.3 y aplicando los métodos de solución se continúa con la siguiente etapa de la metodología, la cual es el acoplamiento de los procesos, la cual se presenta en la siguiente sección. 


\section{Acoplamiento e Implementación}

El modelo matemático del reactor nuclear enfriado con plomo está formado principalmente por el modelo de la potencia neutrónica, modelo de transferencia de calor en el combustible y el termofluido del plomo líquido. Una respuesta en la potencia del reactor se traduce en cambios en las temperaturas del combustible y del plomo, y éstos en la potencia, generando un proceso de retroalimentación. Dada la naturaleza de los procesos neutrónicos, transferencia de calor en el combustible y el termofluido se trata de un acoplamiento multi-físico. Establecer en forma precisa las variables de interacción entre los diferentes procesos es fundamental para codificarlo e implementarlo en una computadora.

\subsection{Acoplamiento}

El acoplamiento multi-físico de los procesos neutrónicos, de transferencia de calor en el combustible y el termofluido es de gran importancia para establecer un análisis a mayor escala del núcleo del reactor nuclear.

En la Figura 6.1 se muestra de manera sencilla el acoplamiento multi-físico. Los procesos neutrónicos y los procesos de transferencia de calor tienen una retroalimentación bidireccional, es decir, la neutrónica genera variables que, en cierta forma, determinan la distribución de temperaturas dentro de la barra de combustible, pero a su vez, estas temperaturas son necesarias para el desarrollo de los procesos neutrónicos. Esta retroalimentación también ocurre entre el combustible y el termofluido, ambos generan y requieren variables del otro proceso. En el caso del acoplamiento entre el termofluido y la neutrónica, solo el primer proceso genera información necesaria para los procesos neutrónicos.

En la Figura 6.2 se muestran las variables que interrelacionan cada fenómeno. Dentro de los procesos neutrónicos se obtienen la potencia neutrónica $[P(t)]$ pero para obtener esta variable se requiere como variables dinámicas las temperaturas del combustible, del encamisado y del refrigerante, las dos primeras son calculadas dentro de los procesos de transferencia de calor. 


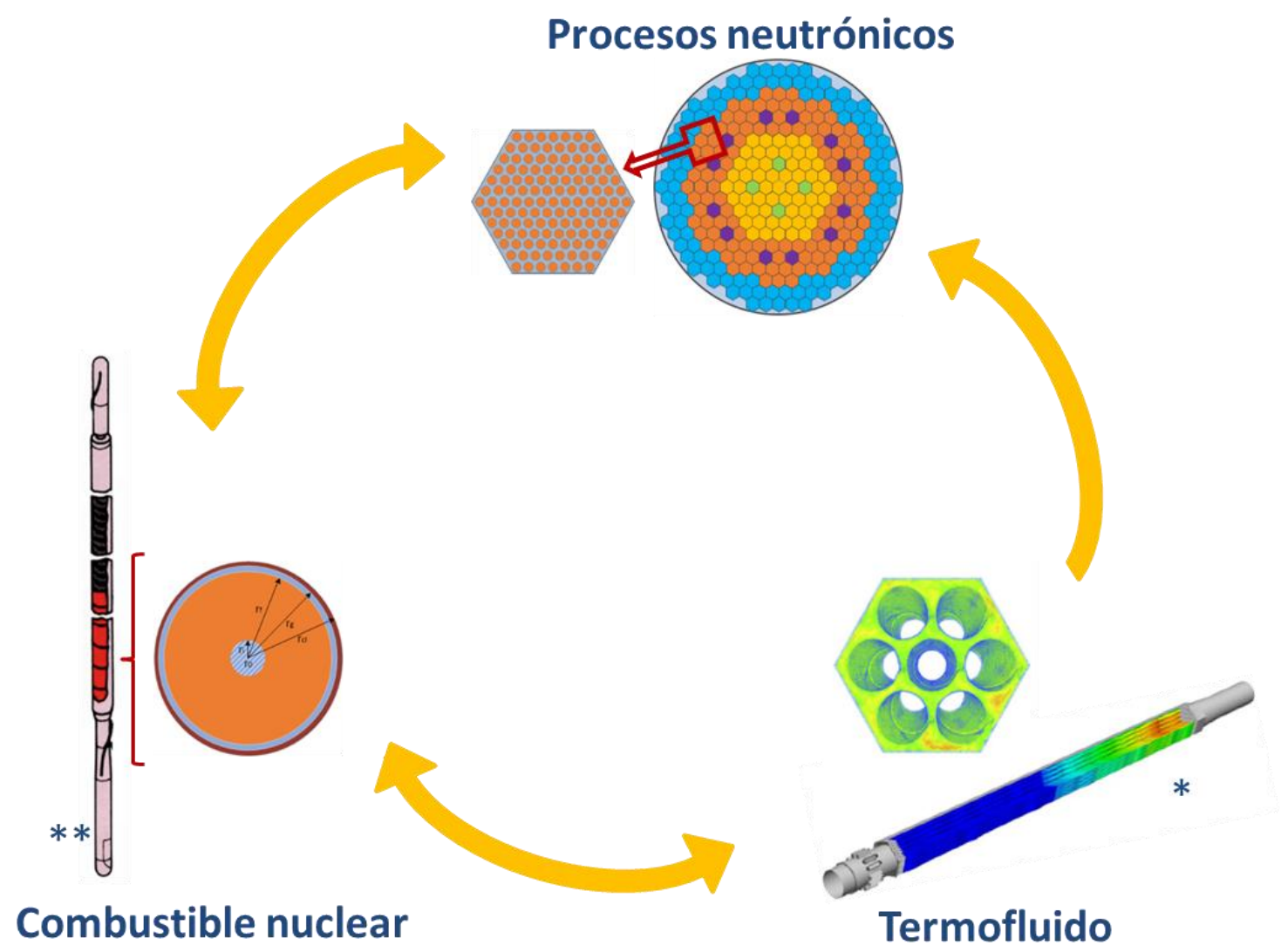

Figura. 6.1. Acoplamiento Multi-físico [* (Roelofs, 2016), ** (Courcelle, 2016)].

La potencia neutrónica está directamente relacionada con la fuente calor $\left[q^{\prime \prime \prime}(t)\right]$, este valor es un parámetro de entrada en el proceso de transferencia de calor dentro de la barra de combustible así como la temperatura del plomo, y como variables de salida en este proceso se tienen las temperaturas del combustible, del gap (holgura) y del encamisado.

En la etapa de procesos del termofluido se necesitan como variables de entrada la temperatura del encamisado (pared), para calcular el calor removido por el refrigerante, también requiere de la temperatura y flujo por unidad de área de la masa a la entrada del núcleo, como resultado se obtiene la temperatura del refrigerante, la cual es requerida para el análisis de los procesos neutrónicos, así como las temperaturas promedio del combustible para el cálculo de la reactividad. 


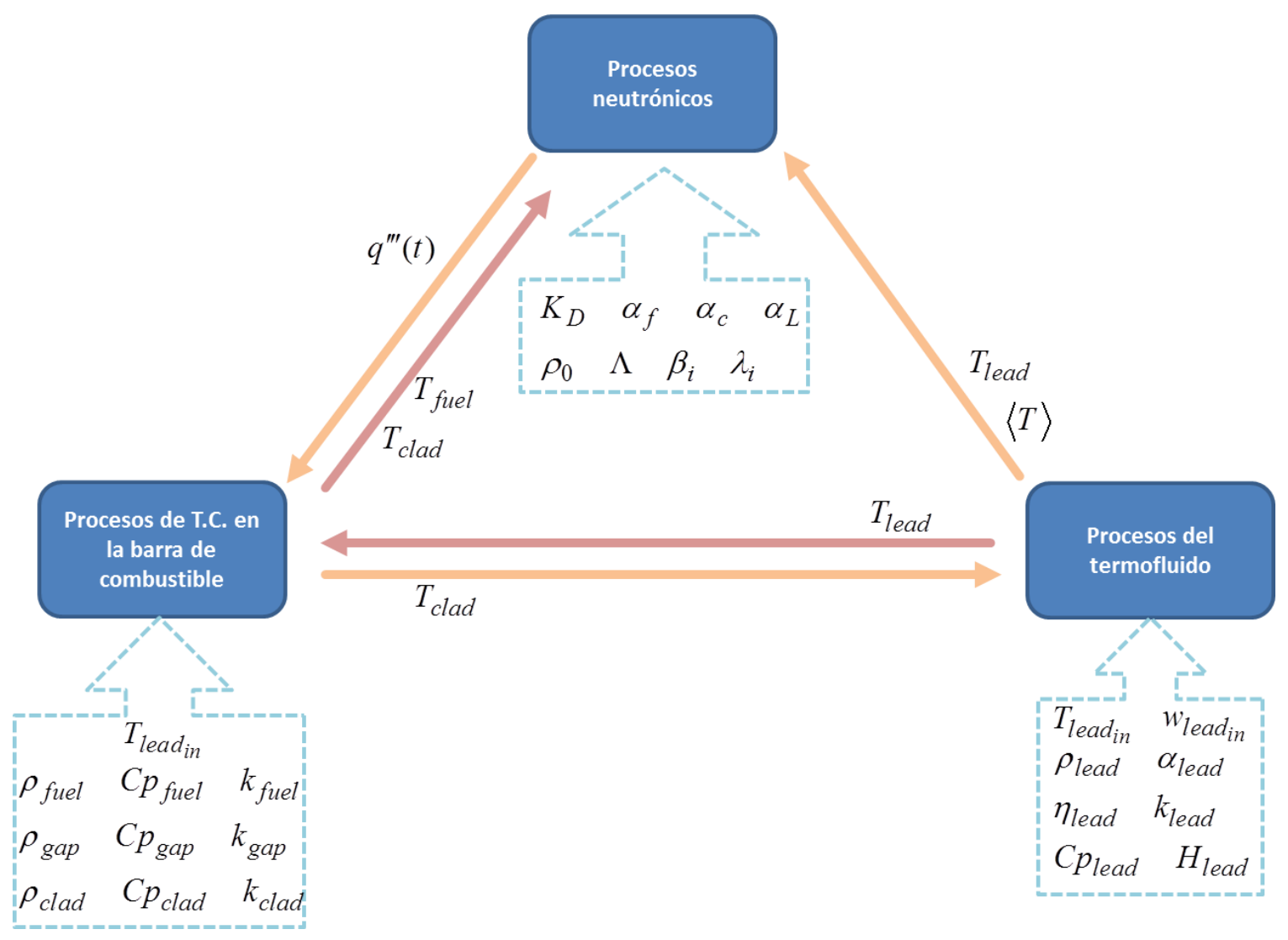

Figura 6.2. Diagrama de variables para el acoplamiento multi-físico.

Esta forma natural de interacción entre procesos multi-físicos y multi-escala, permitió establecer una metodología de acoplamiento para su implementación y pruebas en computadora.

\subsection{Implementación}

En esta sección se describe la implementación de los modelos descritos anteriormente y del acoplamiento dentro del código desarrollado. El código está escrito en Digital Visual FORTRAN.

El programa principal se llama PLOMO_REACTOR y cuenta con 8 rutinas. El programa permite leer los datos de condiciones iniciales, condiciones de frontera de archivos externos .dat, además de poder almacenar la información generada.

Las principales rutinas se describen a continuación:

- PLOMO_REACTOR. Programa principal del código, permite generar resultados a partir de la lectura de información inicial y guardarlos en archivos que contienen la información resultante en un instante. 
- KINETI. Contiene el modelo puntual de la cinética neutrónica así como los datos iniciales para el modelo.

- THLEADR. Rutina que acopla la transferencia de calor en el combustible y el termofluido.

- PROPLEADR. Calcula las propiedades del fluido a partir de ecuaciones constitutivas y la temperatura.

- RODFUEL. Calcula el calor generado en el combustible.

- ENERGIA. Calcula el comportamiento de la temperatura en el termofluido.

- MOMENTO. Solución de la ecuación de cantidad de movimiento y de masa del refrigerante.

- DITUS. Contiene los números adimensionales $\mathrm{Pr}, \mathrm{Re}, \mathrm{Nu}$.

- DPOTKDT. Contiene el método numérico de integración de Euler.

En la Figura 6.3 se presenta la estructura del código por llamadas de las rutinas.

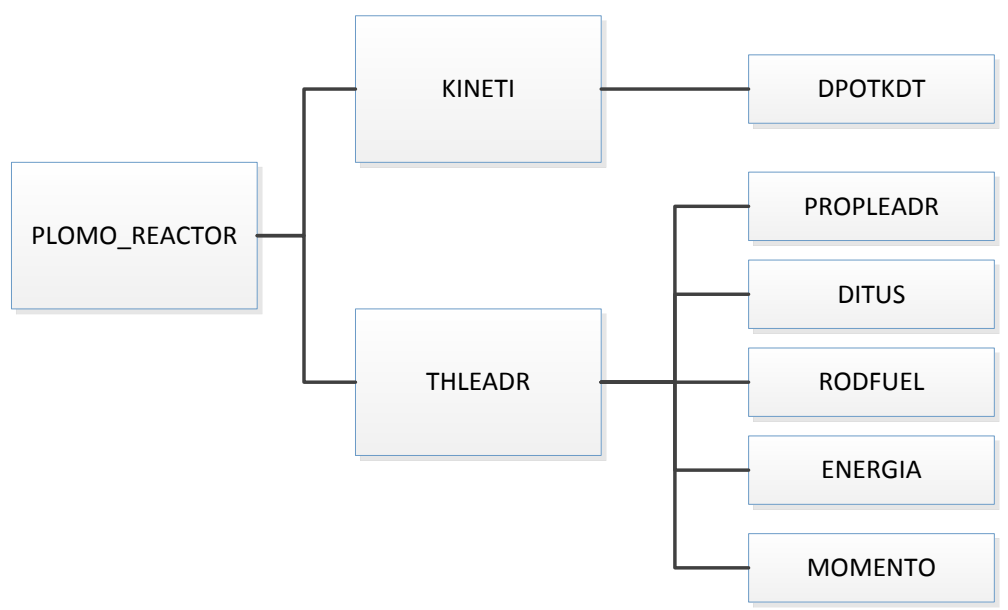

Figura 6.3. Diagrama del código PLOMO_REACTOR por llamadas. 


\section{Experimentos numéricos}

En los Capítulos 5 y 6 se realizó el análisis de los fenómenos que se modelan en este trabajo, así como la etapa de acoplamiento que permite comunicar los procesos y realizar experimentos numéricos en estado estacionario y en estado transitorio del funcionamiento del reactor rápido enfriado con plomo.

En el estudio de reactores nucleares, el análisis de las respuestas que tiene un reactor en estado estacionario y con alguna perturbación es importante desde el punto de vista de seguridad, ya que permite determinar los límites y características de los materiales, así como identificar posibles sistemas de control (por ejemplo, flujo y temperatura del reactor y barras de control).

Al estudiar estos estados de perturbación, también conocidos como transitorios, es necesario conocer: a) si después de la variación producida el reactor alcanza otro estado estacionario, lo que significa que el sistema es estable y, b) si es estable, cómo se comporta el reactor a lo largo de la transición entre los estados estacionarios inicial y final (Glassone y Sesonske, 1990).

En la primera parte de este capítulo se presentan los resultados en estado estacionario, al 100\%, 75\%, $50 \%$ y $25 \%$ de potencia de operación. Posteriormente se presentarán estados transitorios de temperatura de entrada del refrigerante, flujo del refrigerante y reactividad por barras de control.

\subsection{Estado estacionario}

En el estado estacionario se puede observar cuales son las características de operación nominales del reactor, es decir, sin ninguna perturbación.

En la Figura 7.1 se muestra el perfil radial a diferentes alturas de una barra de combustible al $100 \%$ de potencia, la primera sección (FUEL) corresponde al combustible, la siguiente es el gap y la tercera es el encamisado (CLAD). En el inicio de la barra $(z=0)$ se tiene la menor temperatura en todas las secciones, en la parte intermedia $(z=0.36 \mathrm{~m})$ se tiene la mayor temperatura axial, y radialmente es la más alta en la zona del fuel y del gap, sin embargo en la sección del clad es menor que la temperatura en la zona más alta de la barra. Este comportamiento se debe a que la distribución axial de potencia del reactor no es uniforme. 


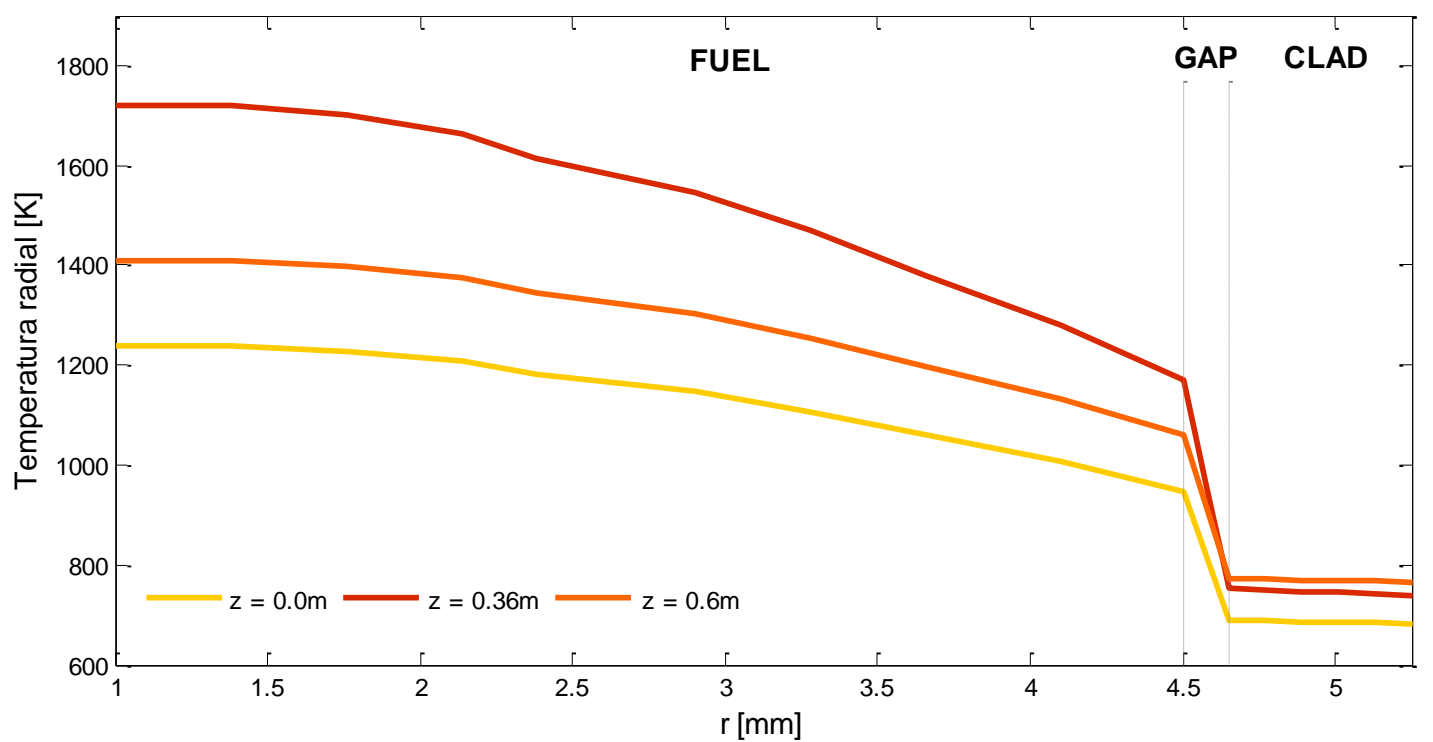

Figura 7.1. Temperatura del combustible (fuel), gap y encamisado (clad) en estado estacionario.

En la Figura 7.2 se presenta el perfil axial de temperaturas en la barra de combustible y del refrigerante, en esta gráfica se puede observar que el perfil del combustible sigue la distribución de potencia axial mostrada en la sección 5.1.

La temperatura del refrigerante va aumentando gracias al flujo de calor absorbido, entra al núcleo a una temperatura de $673.15 \mathrm{~K}$ y sale a $753.15 \mathrm{~K}$.

La Figura 7.3 presenta el campo de temperaturas en la barra de combustible también a 100\% de potencia. Como se puede observar el foco de mayor temperatura se encuentra en el centro en la coordenada axial y al inicio de la coordenada radial. La temperatura más alta es de $1720.06 \mathrm{~K}$ y la más baja de $682.35 \mathrm{~K}$, la cual se ubica en el inicio de la barra en el encamisado.

La variación de la potencia genera una variación de los perfiles de temperatura, debido a la relación que existe entre el proceso neutrónico y el de transferencia de calor. A continuación se presentan los perfiles de temperatura en estado estacionario a $25 \%, 50 \%$ y $75 \%$ de potencia y en la posición axial con mayor temperatura $(z=0.36 m)$. 


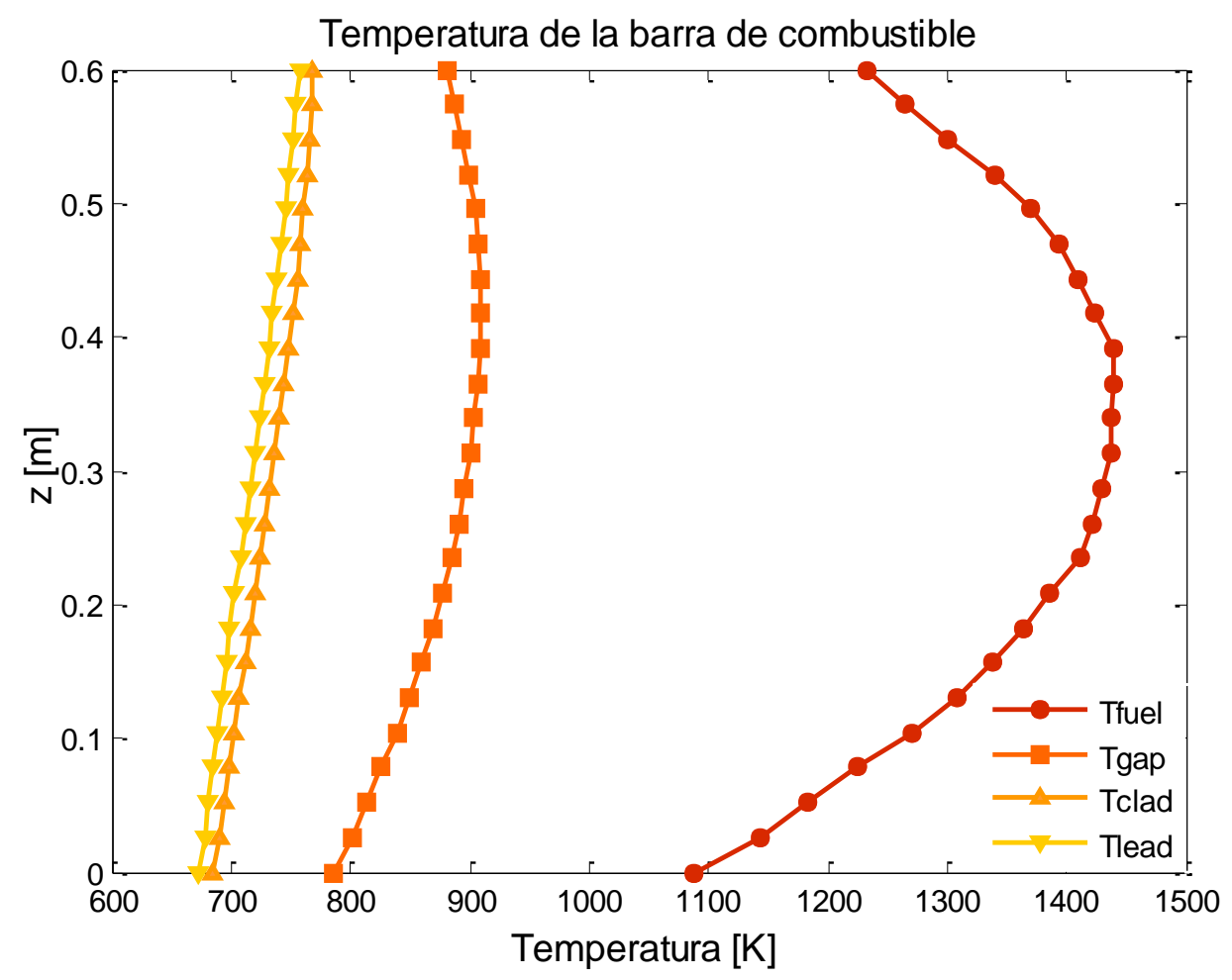

Figura 7.2. Perfil axial de temperatura del combustible (fuel), gap encamisado (clad) y refrigerante (lead) en estado estacionario a $100 \%$ de potencia.

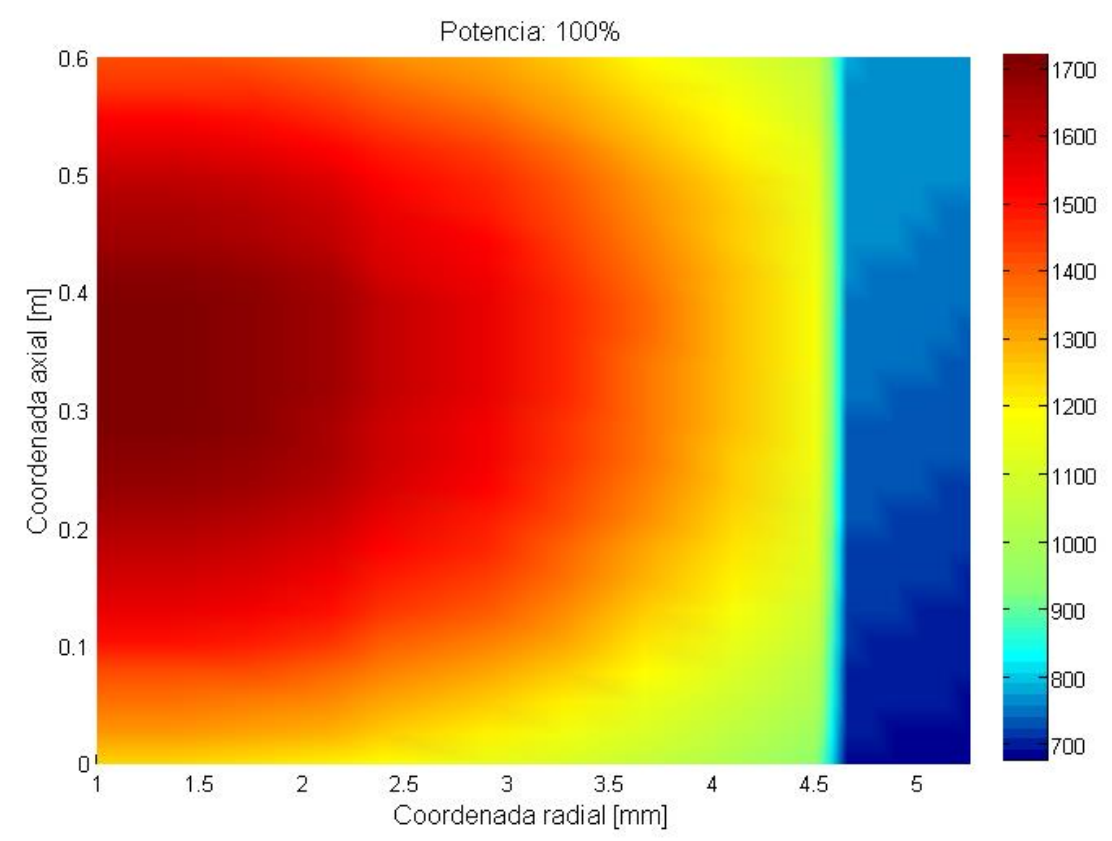

Figura 7.3. Distribución de temperatura en la coordenada radial y axial de la barra de combustible a 100\% de potencia. 
La Figura 7.4 muestra los perfiles de temperatura de la barra de combustible a las potencias antes mencionadas, en este caso a menor potencia, la temperatura radial disminuye. En el caso del 100\% la diferencia entre la temperatura inicial y final de cada sección es mayor que en potencias menores. En la Tabla 7.1 se muestran estas diferencias de temperatura entre cada sección y a diferentes potencias.

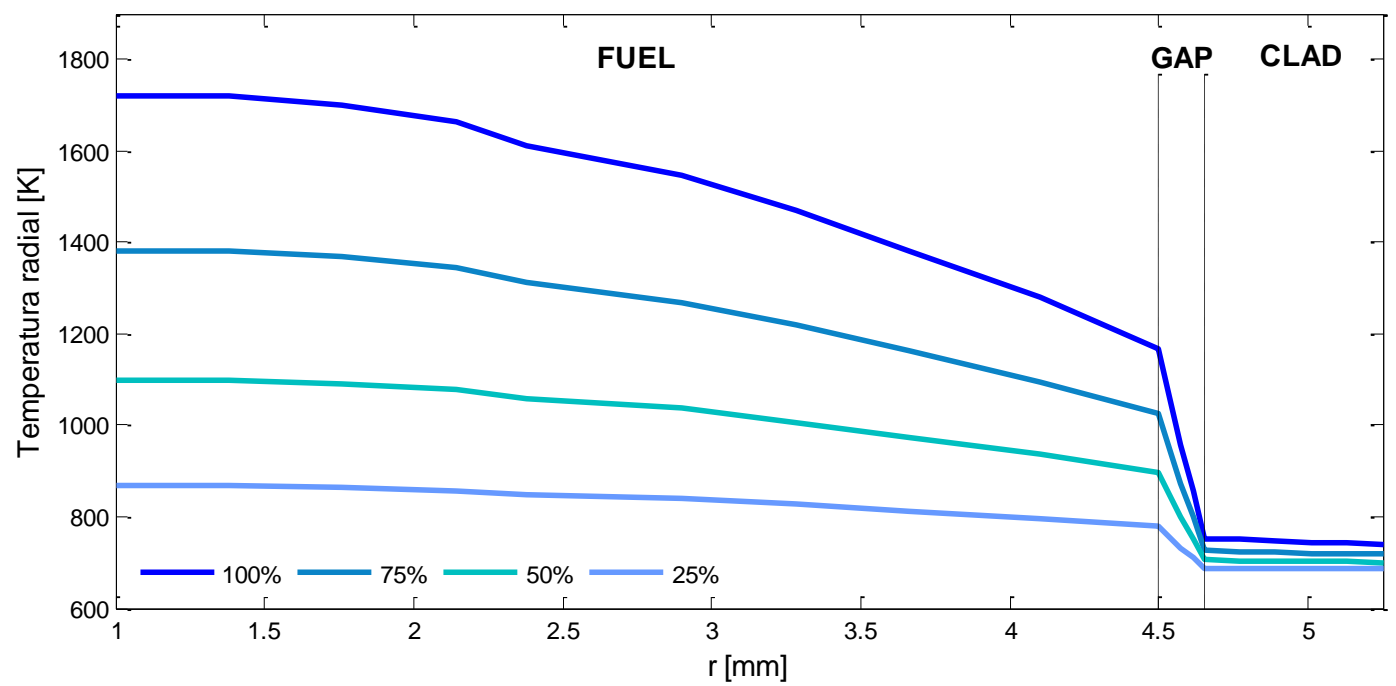

Figura 7.4. Distribución radial de temperatura en la barra de combustible a diferentes potencias de operación a $0.36 \mathrm{~m}$.

Tabla 7.1. Diferencia de temperatura radial inicial y final de las secciones de la barra.

\begin{tabular}{ccccc}
\hline Potencia & $\Delta$ T- Fuel & $\Delta$ T- Gap & $\Delta$ T- Clad & $\Delta$ T -Total \\
\hline $100 \%$ & 551.18 & 417.01 & 11.65 & 979.84 \\
\hline $75 \%$ & 355.36 & 298.34 & 8.14 & 661.84 \\
\hline $50 \%$ & 203.77 & 189.74 & 5.07 & 398.58 \\
\hline $25 \%$ & 87.91 & 90.69 & 2.38 & 180.98 \\
\hline
\end{tabular}

En la Figura 7.5 se muestran los perfiles de temperatura de cada una de las secciones de la barra de combustible a diferentes potencias de operación, la gráfica a) muestra la temperatura del combustible, b) la temperatura en el gap, c) temperatura en el encamisado y d) temperatura en el refrigerante.

En el caso del combustible, el gap y el encamisado, la temperatura al inicio de la barra es diferente para cada potencia, esto se debe a que la distribución de temperatura depende del calor generado por la fuente. Entonces, a menor potencia se tendrá menor calor generado y por lo tanto una temperatura menor. Para el refrigerante no ocurre lo mismo, ya que la temperatura de entrada de éste al núcleo es un valor establecido, $480^{\circ} \mathrm{C}(753.15 \mathrm{~K})$ y la temperatura de salida varía de acuerdo al calor absorbido. Para $100 \%$ 
de potencia, la temperatura de salida es de $753.6 \mathrm{~K}$, para $75 \%$ es de $729.15 \mathrm{~K}$ y para $50 \%$ y $25 \%$ es $707.02 \mathrm{~K}$ y $688.66 \mathrm{~K}$ respectivamente.
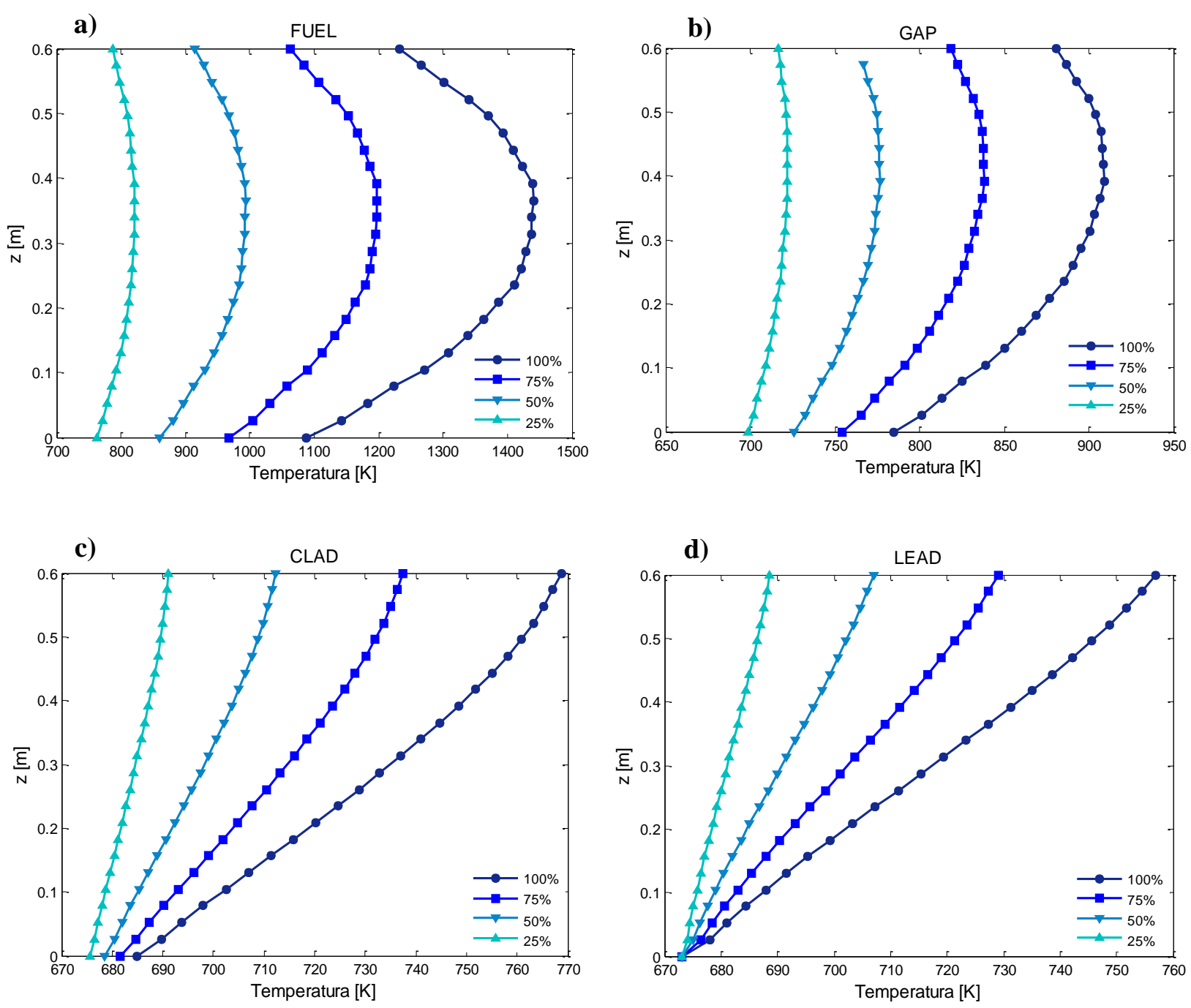

Figura 7.5. Perfil axial de temperatura a 100\%, 75\%, 50\% y 25\% de potencia de la sección a) combustible, b) gap, c) clad y d) refrigerante.

En la Figura 7.6 se muestra la distribución radial y axial de temperatura de la barra de combustible para $25 \%, 50 \%, 75 \%$ y $100 \%$ de potencia, las cuales se obtuvieron con la posición de las barras de control. Como se puede observar en esta Figura, a una potencia de 25\%, el campo de temperaturas es la menor magnitud de los cuatro casos estudiados, para esta potencia la temperatura más alta es de $866.49 \mathrm{~K}$ y la menor es de $675.11 \mathrm{~K}$, en el caso de $50 \%$, la más alta es de $1098.78 \mathrm{~K}$ y la menor de $677.35 \mathrm{~K}$, y para $75 \%$ de potencia se tienen $1379.77 \mathrm{~K}$ y $679.76 \mathrm{~K}$ mayor y menor, respectivamente. 

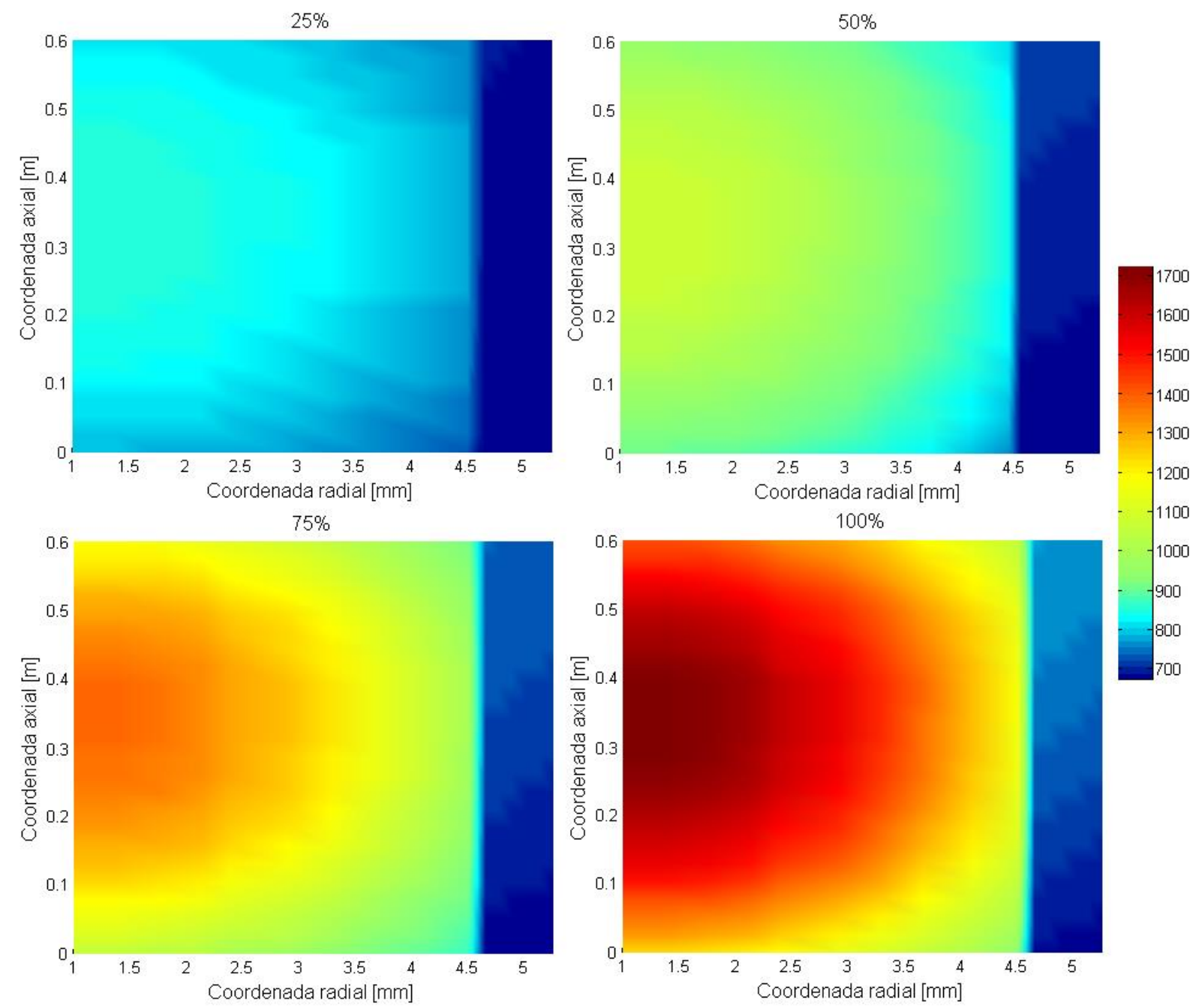

Figura 7.6. Campo de temperaturas de temperatura en una barra de combustible a $25 \%, 50 \%, 75 \%$ y $100 \%$ de potencia.

\subsection{Estado transitorio}

Un transitorio se define como un evento en una planta nuclear, el cual procede de un estado normal a un estado anormal (Moshkbar-Bakhshayesh y Ghofrani, 2013). Este evento es producido cuando existe una perturbación que genere un cambio en los parámetros de operación.

Estos cambios pueden ser en la potencia, reactividad, presión, en el sistema primario o secundario de enfriamiento (United States Nuclear Regulatory Commission, 2016). El estudio de estados transitorios sirve para estudiar el tiempo de respuesta del reactor y su estabilidad, así como las posibles aplicaciones de control. 
En este trabajo se realizó la experimentación numérica con tres perturbaciones al reactor: (1) cambio de la temperatura del plomo refrigerante a la entrada del núcleo del reactor, (2) cambio de la velocidad de entrada del refrigerante y (3) cambio de reactividad. El procedimiento es sencillo y directo, i.e., a los 5 segundos del tiempo transcurrido de simulación se introduce la perturbación simulada.

\subsubsection{Variación de la temperatura}

Este transitorio corresponde a la variación de la temperatura del refrigerante a la entrada del núcleo, el diseño del reactor establece que la temperatura del reactor es de $400^{\circ} \mathrm{C}(673.15 \mathrm{~K})$, para efectos de este trabajo se realizaron las simulaciones cambiando la temperatura a $653 \mathrm{~K}, 663 \mathrm{~K}, 683 \mathrm{~K}$ y $693 \mathrm{~K}$.
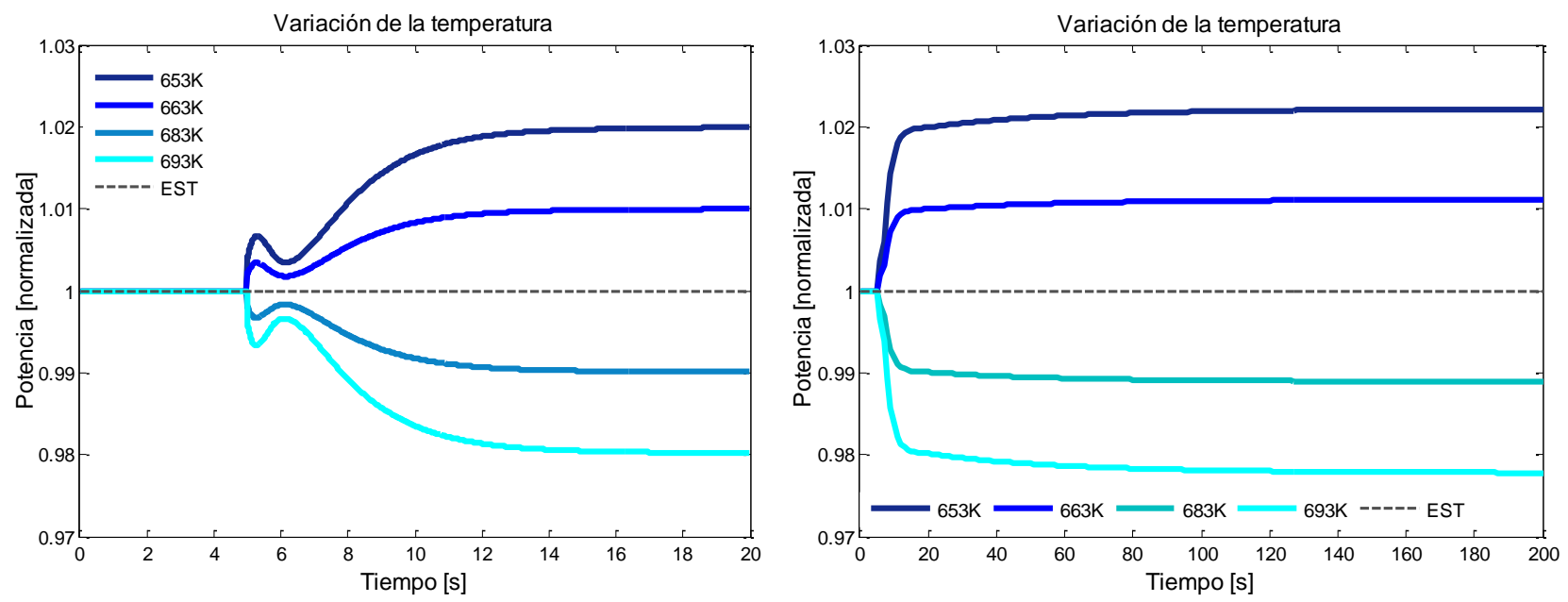

Figura 7.7. Potencia normalizada durante una perturbación en la temperatura del refrigerante.

En la Figura 7.7 se presenta el efecto del cambio de temperatura en la potencia (normalizada) del reactor. La gráfica del lado izquierdo de esta Figura corresponde a los primeros 20 segundos de simulación (tiempos cortos), y en la de la derecha corresponde a tiempos largos de simulación donde se puede observar que se alcanza un nuevo estado estacionario.

Se observa que los primeros cuatro segundos el reactor está en estado estacionario y en el segundo 5 inicia el transitorio. De acuerdo con estos resultados, para temperaturas mayores de entrada al núcleo respecto a las condiciones iniciales $(683 \mathrm{~K}$ y $693 \mathrm{~K})$, la potencia disminuye. Para temperaturas menores $(653$ y $663 \mathrm{~K})$ el comportamiento es inverso, i.e., la potencia aumenta. Esto se debe al efecto de reactividad ya que cuando se genera un cambio de temperatura del refrigerante las temperaturas promedio del combustible, gap y encamisado cambian y la reactividad por efecto Doppler, de expansión del combustible, del gap y del encamisado también sufren un cambio, generando así una nueva reactividad total (véase ecuación 5.25). 
En la Figura 7.8 se muestra el cambio de reactividad total en los primeros 20 segundos de simulación, así como la contribución de cada reactividad para una temperatura de entrada del refrigerante. A partir de los 5 segundos, todas las reactividades, excepto la de barras de control, sufren un cambio. Para $653 \mathrm{~K}$ la reactividad por efecto Doppler se hace más negativa, en cambio cuando la temperatura es de $693 \mathrm{~K}$ el efecto Doppler es menos negativo. El efecto y significado físico de este comportamiento se debe a que a menores temperaturas el efecto Doppler es menor y permite mayores reacciones de fisión y por lo tanto favorece el aumento de potencia, y a mayores temperaturas las reacciones de fisión disminuyen por este efecto.
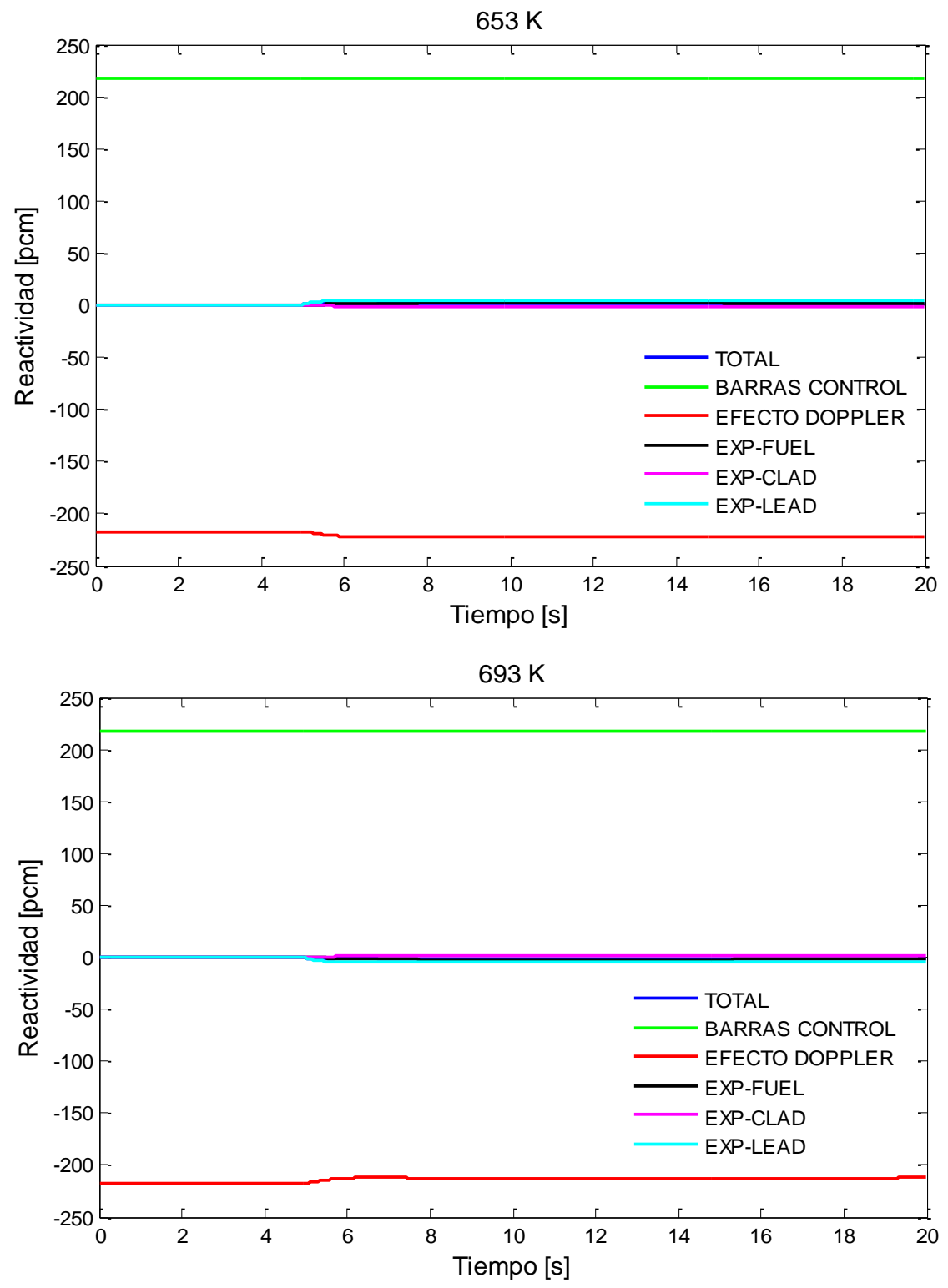

Figura 7.8. Reactividades durante la perturbación de la temperatura. 
En la Figura 7.9 se presenta un acercamiento de las gráficas mostradas anteriormente, con la finalidad de analizar las reactividades. Como se puede observar, para $653 \mathrm{~K}$ la reactividad por expansión del refrigerante aumenta, al igual que la de expansión del combustible y la de expansión del encamisado disminuye, en este caso la contribución mayor es la de la expansión del refrigerante, lo que hace que la reactividad total sea positiva, y por lo tanto favorezca el aumento de potencia por este fenómeno.

En el caso de 693 K, la contribución mayor es también la de expansión del refrigerante, pero en este caso es negativa, por lo que, la reactividad total es negativa, y propicia una disminución de potencia.
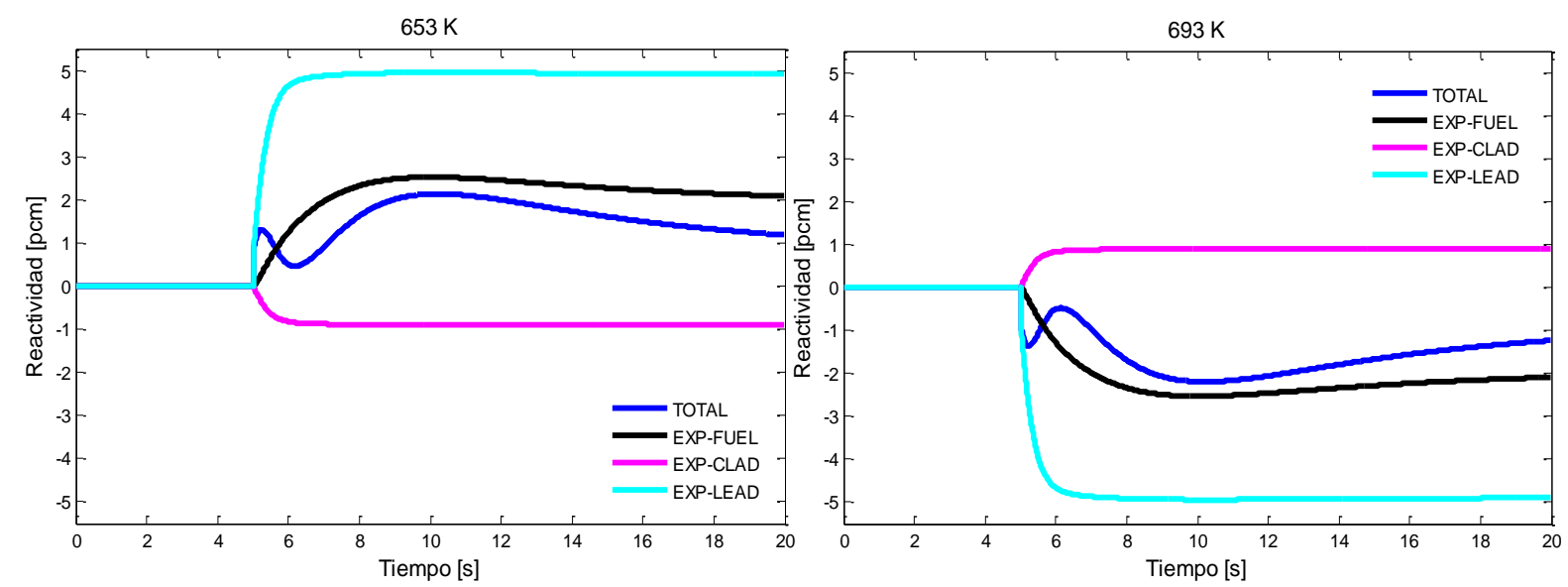

Figura 7.9. Acercamiento del comportamiento de reactividades en una perturbación de temperatura.

Entonces, si la temperatura de entrada del refrigerante disminuye, la reactividad total aumenta y al tener una reactividad positiva la potencia del reactor aumenta hasta estabilizarse nuevamente y si la temperatura aumenta, el efecto es inverso.

En la Figura 7.10 se puede observar el cambio de reactividad total para las cuatro temperaturas analizadas, en donde, como se mencionó anteriormente, a mayor temperatura del plomo la reactividad disminuye y a menor temperatura, aumenta. A partir de que entra el transitorio la reactividad empieza a cambiar y buscando la estabilidad del reactor llega a cero en aproximadamente 130s.

De la misma forma que ocurre con la potencia, la concentración de precursores sufre el mismo cambio, como se observa en la Figura 7.11. 

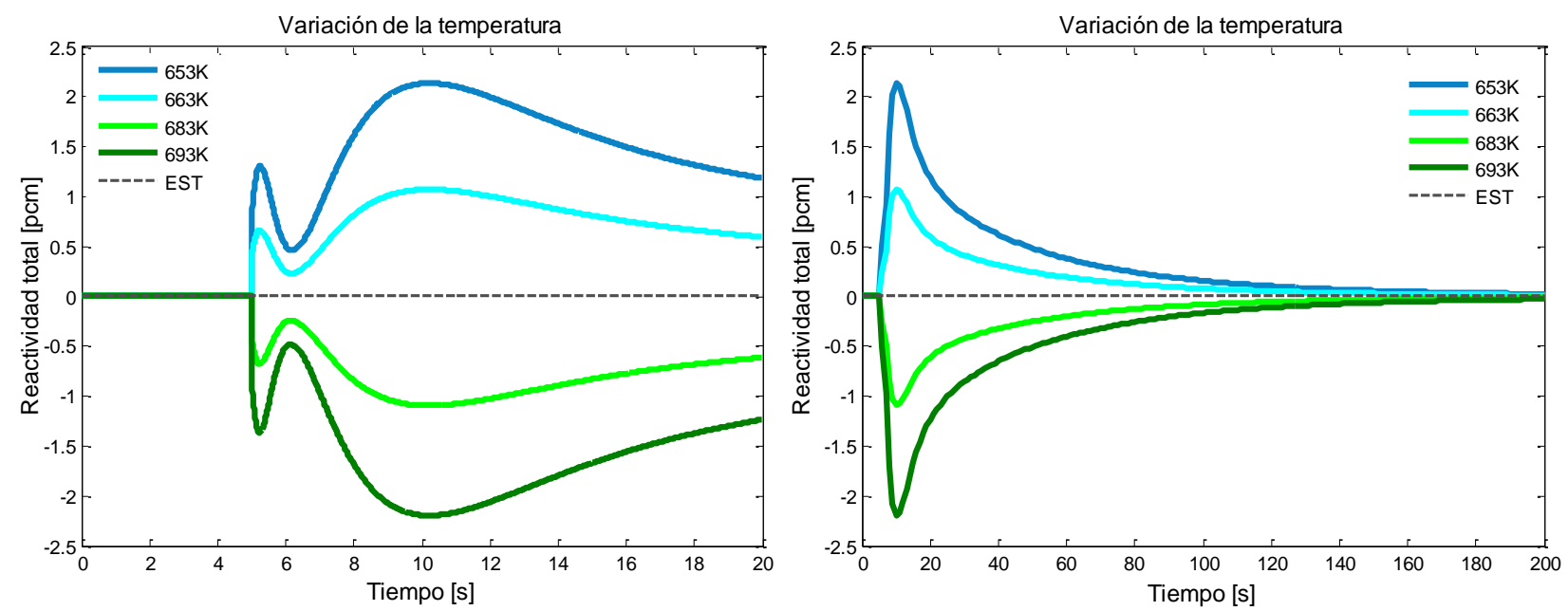

Figura 7.10. Reactividad total durante una perturbación en la temperatura del refrigerante.
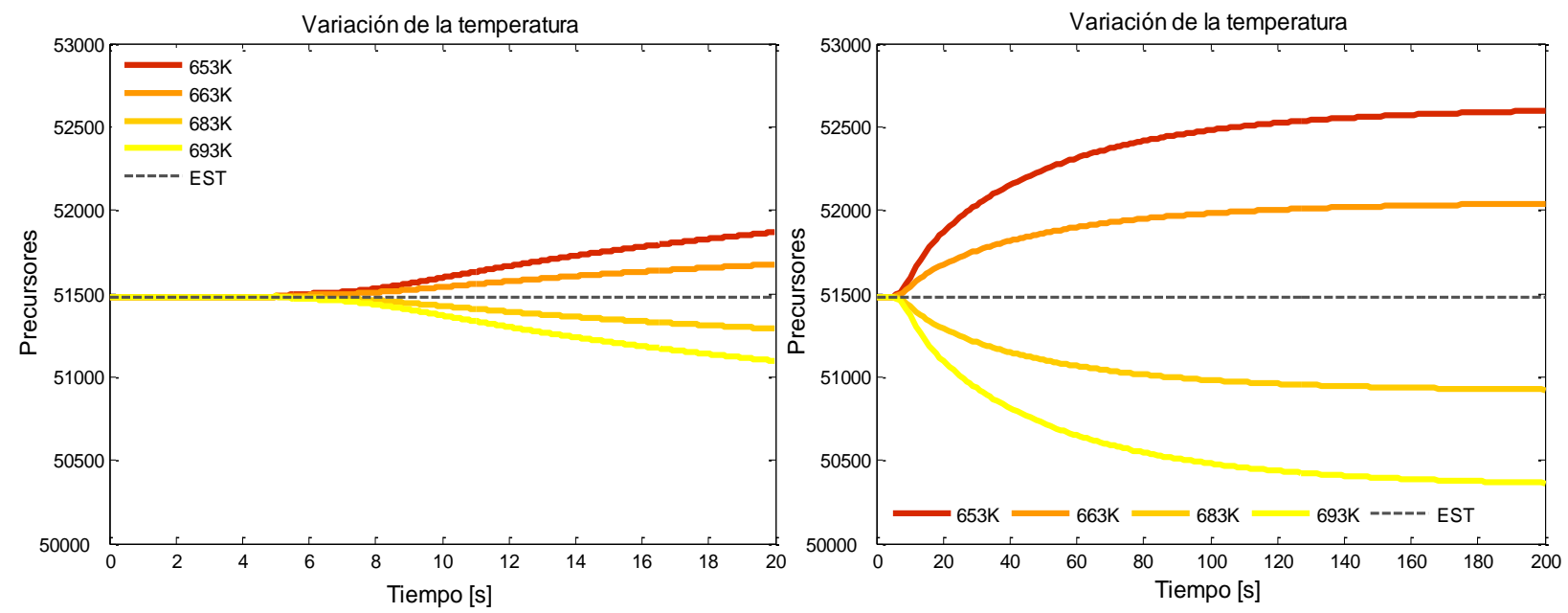

Figura 7.11. Generación de percusores durante una perturbación en la temperatura del refrigerante.

Como se puede observar, la temperatura a la entrada del núcleo del reactor rápido enfriado con plomo es un parámetro importante para el control de potencia.

\subsubsection{Variación de la velocidad}

El segundo transitorio generado fue el cambio de velocidad del refrigerante a la entrada del núcleo. La velocidad nominal es de $2 \mathrm{~m} / \mathrm{s}$, las variaciones propuestas son $1 \mathrm{~m} / \mathrm{s}, 1.5 \mathrm{~m} / \mathrm{s}, 2.5 \mathrm{~m} / \mathrm{s} \mathrm{y} 3 \mathrm{~m} / \mathrm{s}$.

En este caso, el efecto del cambio de velocidad es más representativo en las velocidades menores a la nominal (Figura 7.12), esto debido al cambio de reactividad. Si la velocidad disminuye, la potencia 
disminuye; debido a que la temperatura del refrigerante aumenta, así como el combustible, gap y encamisado, generando así una disminución de la reactividad.
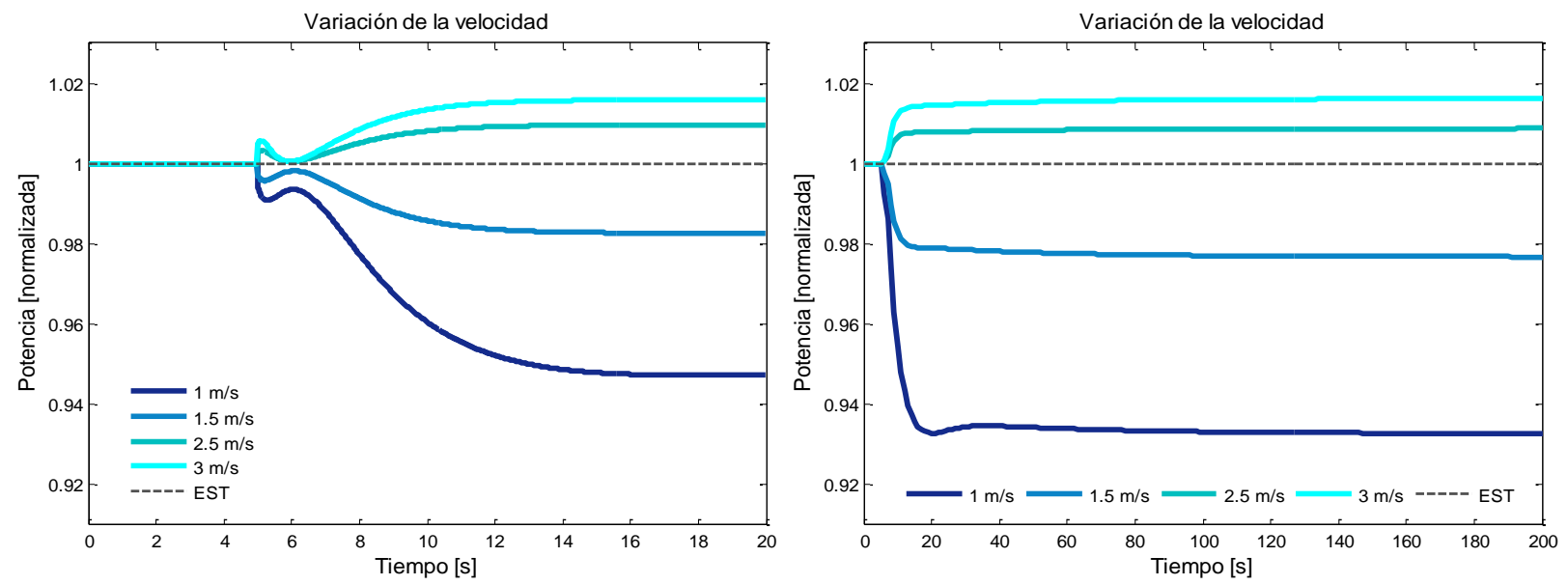

Figura 7.12. Potencia (normalizada) durante una perturbación en la velocidad del refrigerante.

En la Figura 7.13 se puede observar el efecto de este transitorio en la reactividad. Al igual que la potencia, el efecto es mayor cuando la velocidad disminuye. Durante los primeros 20s se tiene un gran cambio en la reactividad, y después la reactividad va disminuyendo para llegar nuevamente al estado estacionario.

En el caso contrario, la reactividad aumenta y de igual forma la potencia, aunque el efecto es menor. En la Figura 7.14 se observa la contribución de cada una de las reactividades, en este caso, la reactividad por efecto Doppler es mayor para velocidades menores a la nominal.
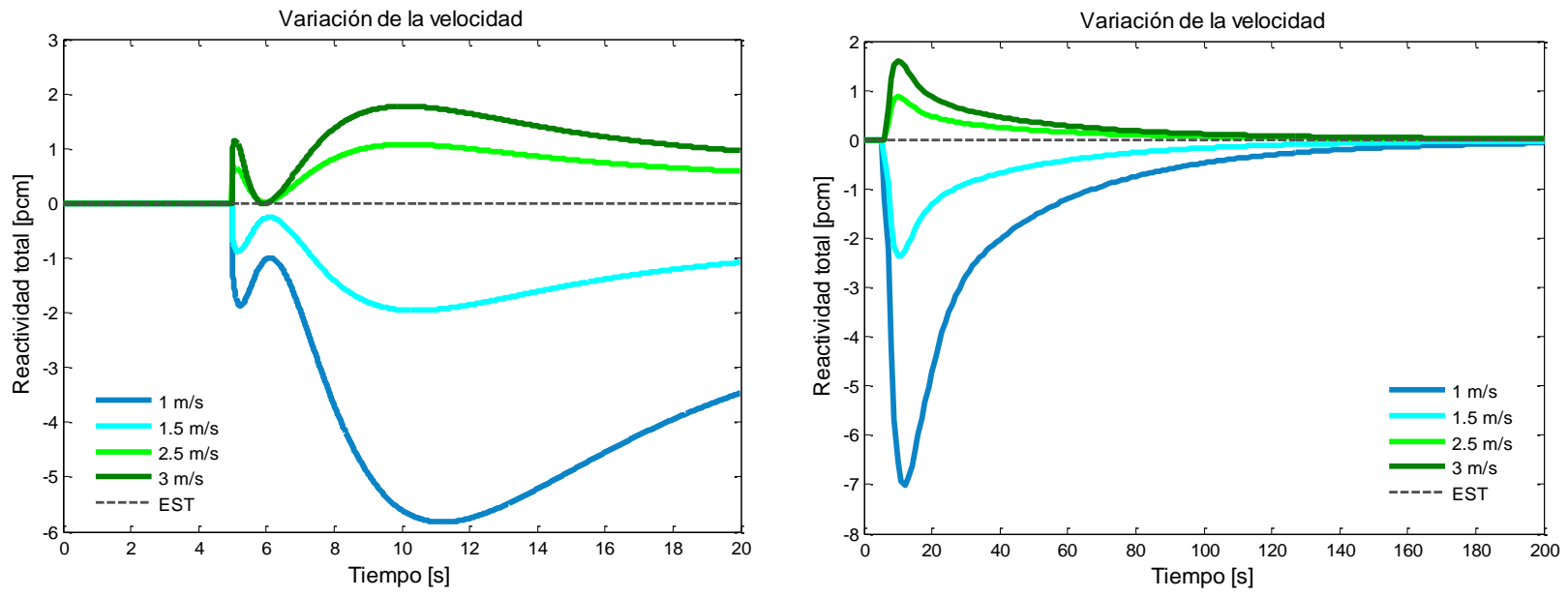

Figura 7.13. Reactividad total durante una perturbación velocidad del refrigerante. 

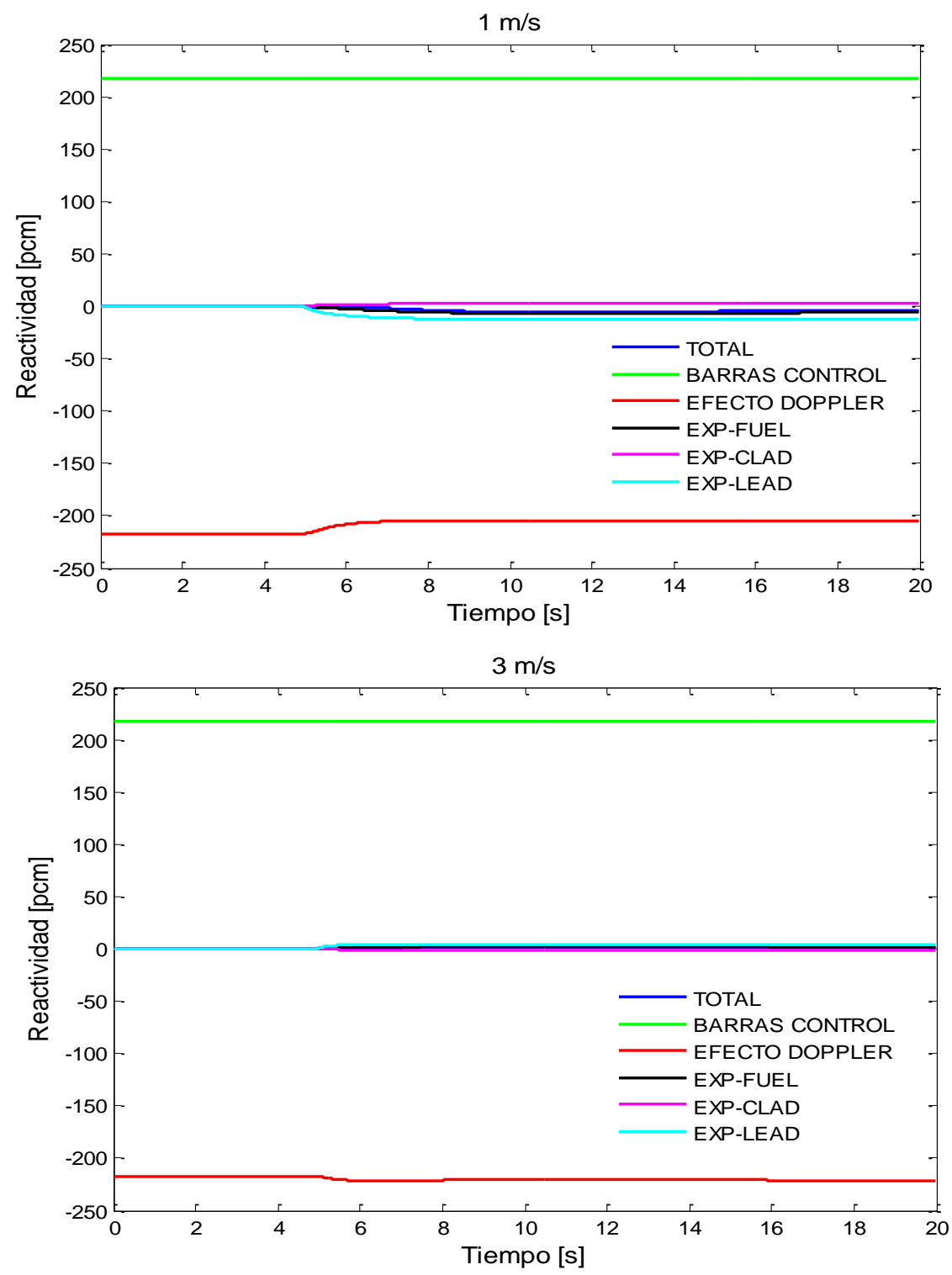

Figura 7.14. Reactividades en una perturbación de velocidad de refrigerante.

Para este transitorio, la reactividad por efecto de expansión del refrigerante es la que más aportación tiene, seguida por la expansión del combustible y al final la expansión del encamisado (Figura 7.15). Al iniciar el transitorio, la reactividad total va cambiando por efecto del cambio de las reactividades $\mathrm{y}$ aproximadamente en el segundo 12 comienza con una tendencia a ser cero.

En el caso de la concentración de precursores (Figura 7.16), hay una disminución cuando la velocidad disminuye, y aumenta cuando la velocidad aumenta, sin embargo, el efecto es, al igual que en el caso de la potencia, más grande a menores velocidades de refrigerante. 

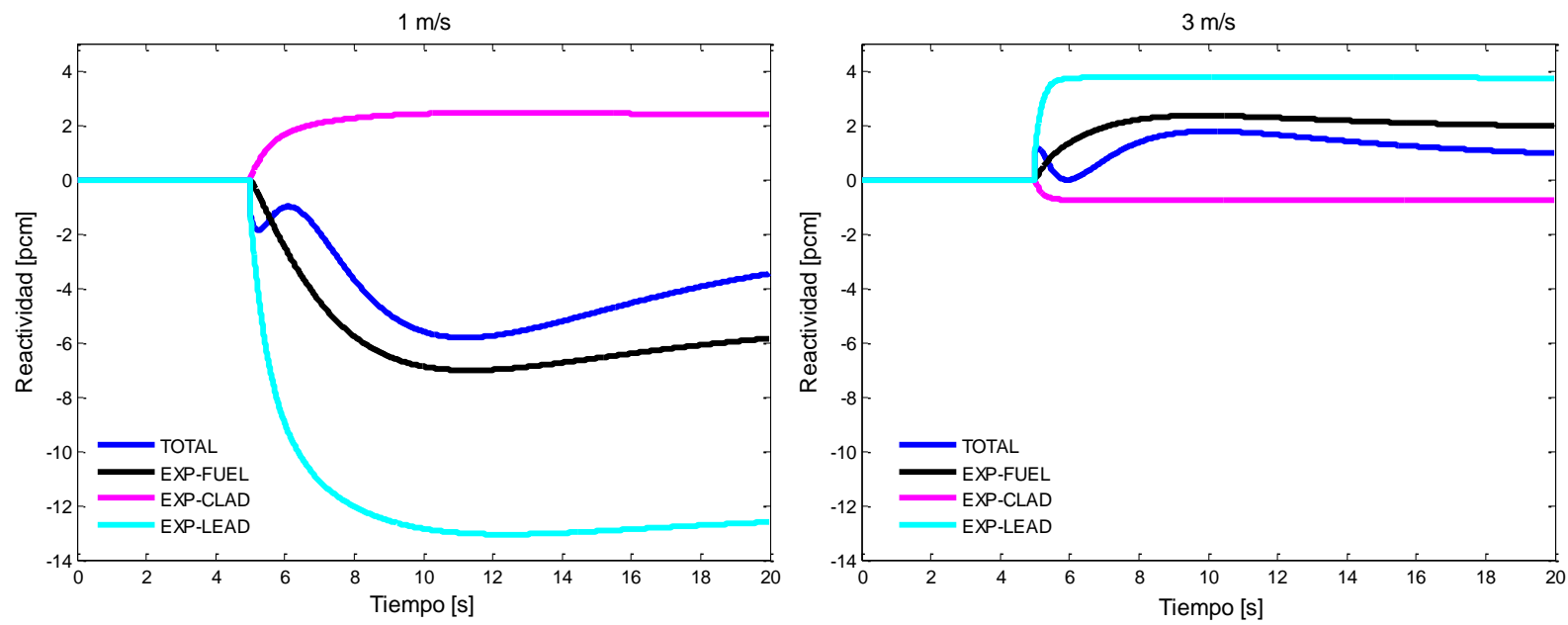

Figura 7.15. Acercamiento del comportamiento de reactividades en una perturbación de velocidad del refrigerante.
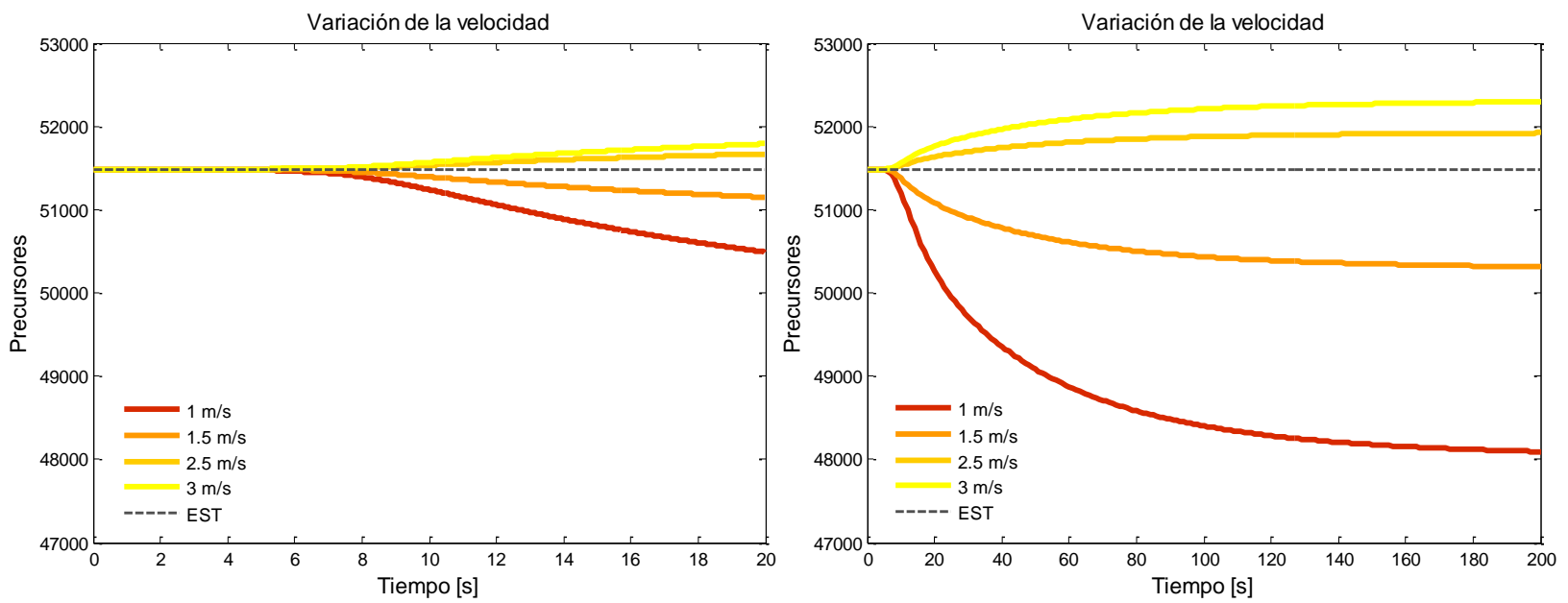

Figura 7.16.Concentración de precursores durante una perturbación en la velocidad del refrigerante

\subsubsection{Variación de la reactividad}

La variación de la reactividad es otro de los transitorios estudiados en este trabajo, esta perturbación puede ser generada por inserción o extracción de la barra de control, se consideró que la reactividad cambia de $+20 \mathrm{pcm},-20 \mathrm{pcm},+10 \mathrm{pcm}$ y $-10 \mathrm{pcm}$, se consideran estos cambios y que se simula un Unprotected Transient of Over Power . Al igual que los transitorios anteriores, el cambio de reactividad ocurre en los primeros 5 segundos.

En la Figura 7.17 se muestra el efecto del cambio de reactividad en la potencia, para cambios positivos de reactividad, la potencia aumenta y para cambios negativos, esta disminuye. 

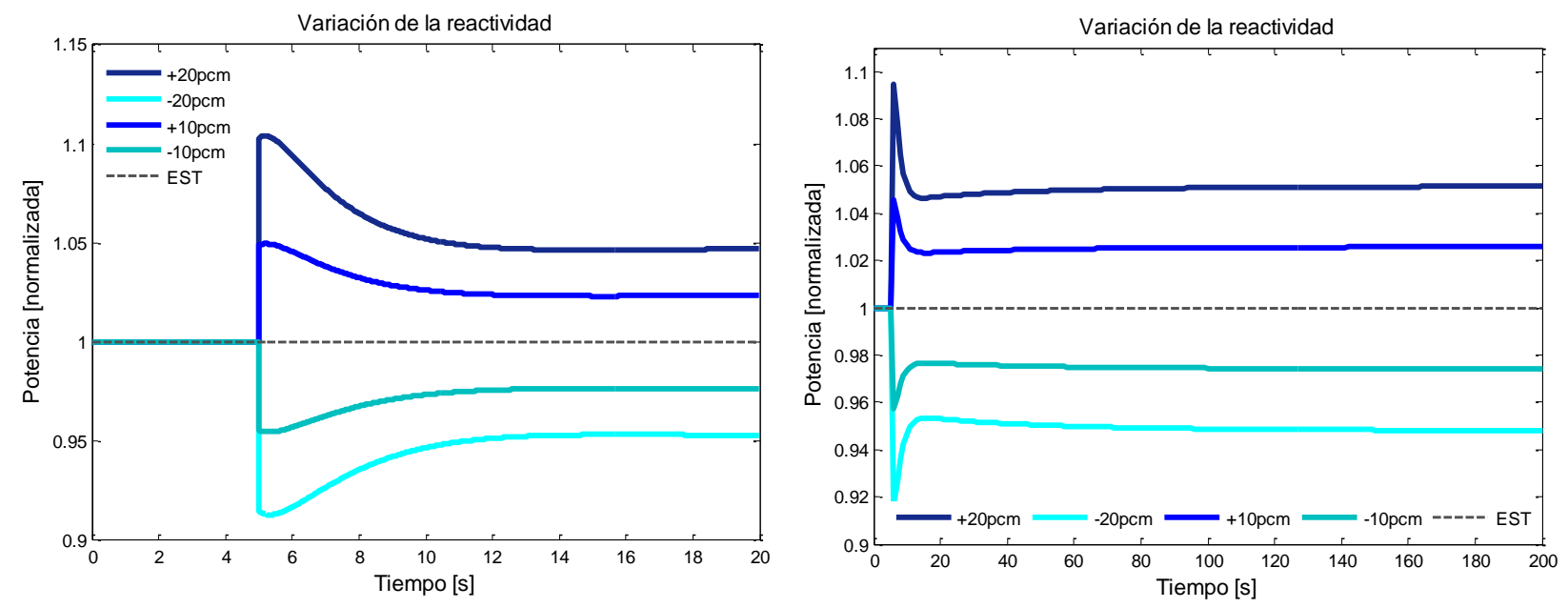

Figura 7.17. Potencia (normalizada) durante la perturbación de reactividad.
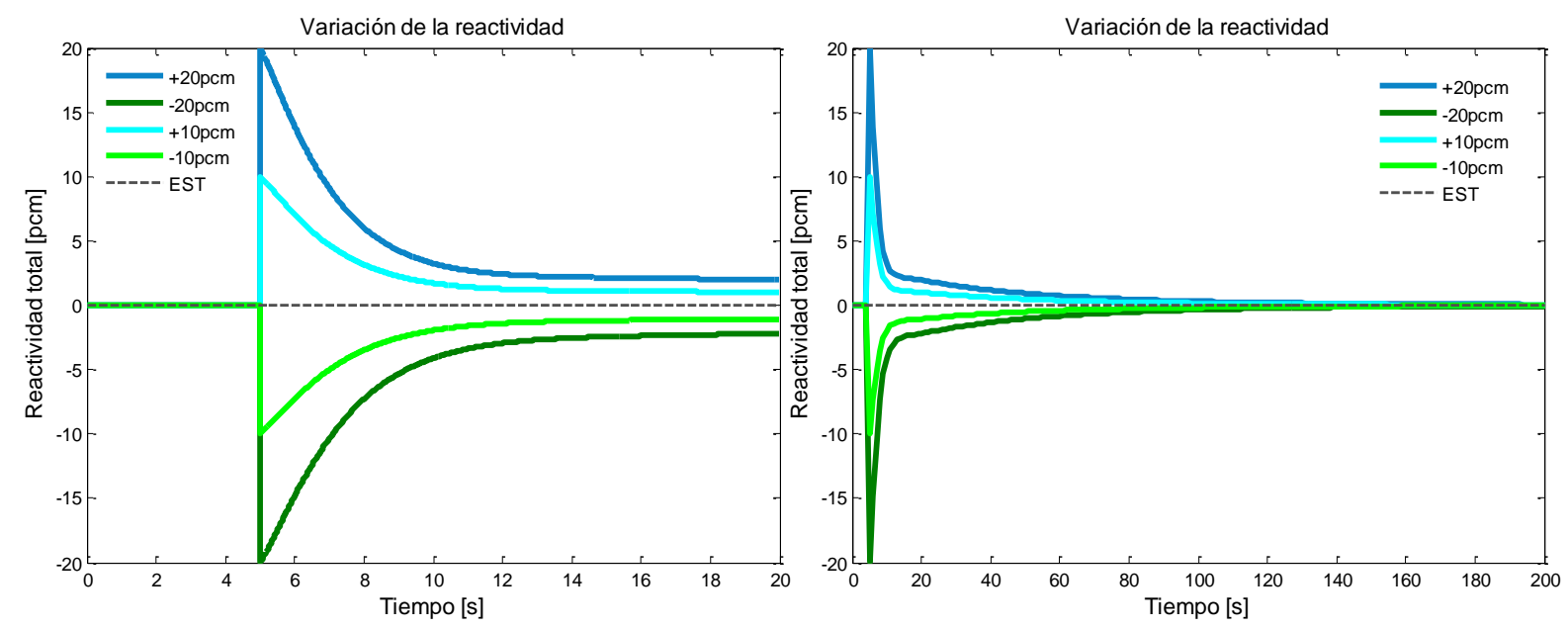

Figura 7.18. Reactividad total durante la perturbación de la reactividad.

En la Figura 7.18 se observa la reactividad total, a los 5 segundos comienza el transitorio y se observa que el máximo o mínimo de la gráfica toma el valor de la perturbación, para después volver a un estado estacionario (reactividad total igual a cero) en aproximadamente $120 \mathrm{~s}$.

La contribución de cada una de las reactividades se puede observar en la Figura 7.19. En este caso, a diferencia de los transitorios anteriores, la reactividad por barras de control también cambia, ya que es aquí en donde se modifica el valor de la reactividad, la reactividad por efecto Doppler disminuye al aumento del valor de barras de control. 

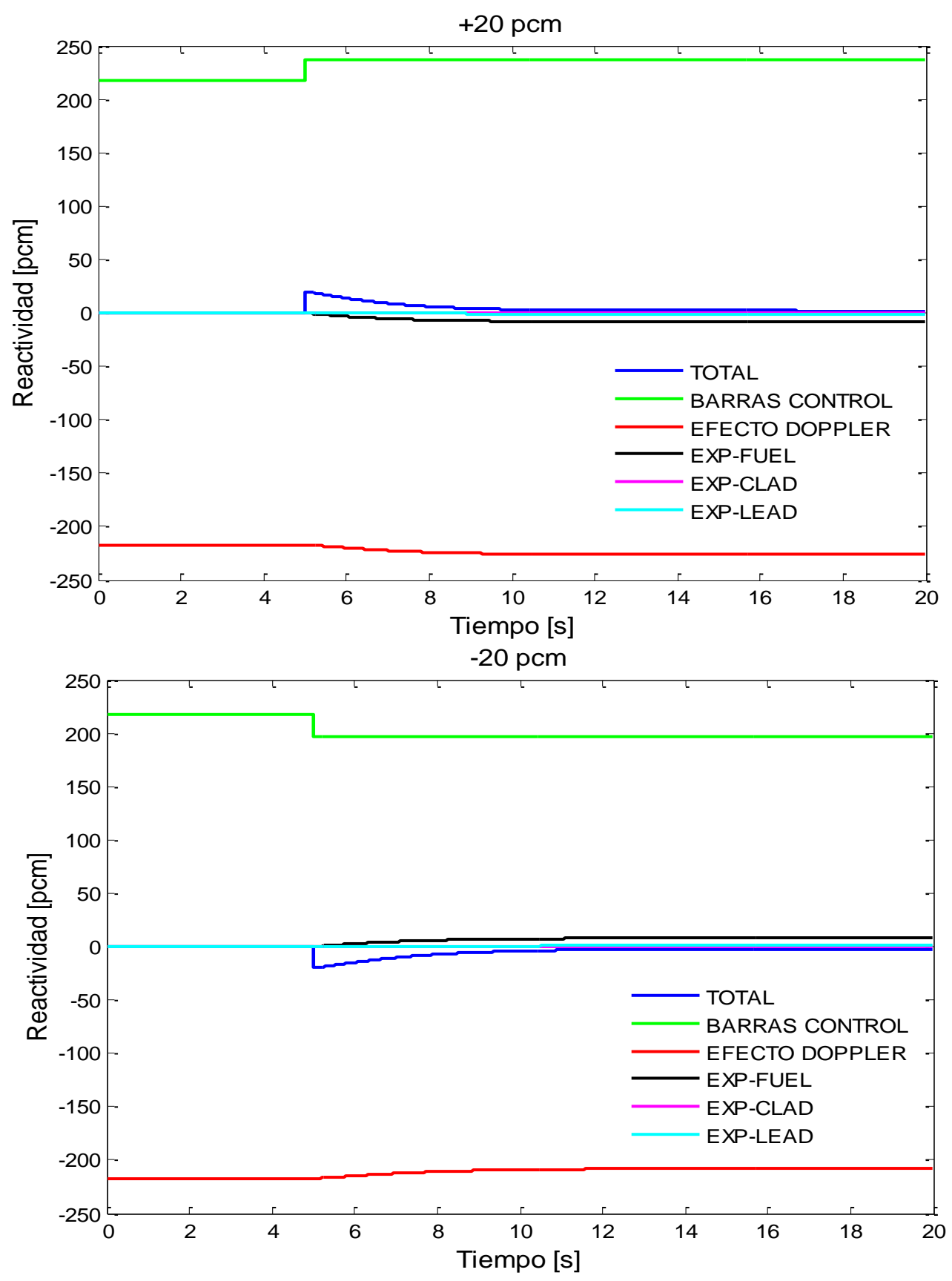

Figura 7.19. Acercamiento del comportamiento de reactividades en una perturbación de reactividad.

En la Figura 7.20 se observa como la reactividad total toma el valor de la perturbación en el segundo 5, para el caso de +20 pcm la reactividad por expansión del combustible disminuye al igual que por efecto Doppler, al contrario que los transitorios anteriores, por expansión del encamisado aumenta un poco y la de expansión del refrigerante disminuye en menor magnitud. Para el caso de -20 pcm los efectos son inversos. 

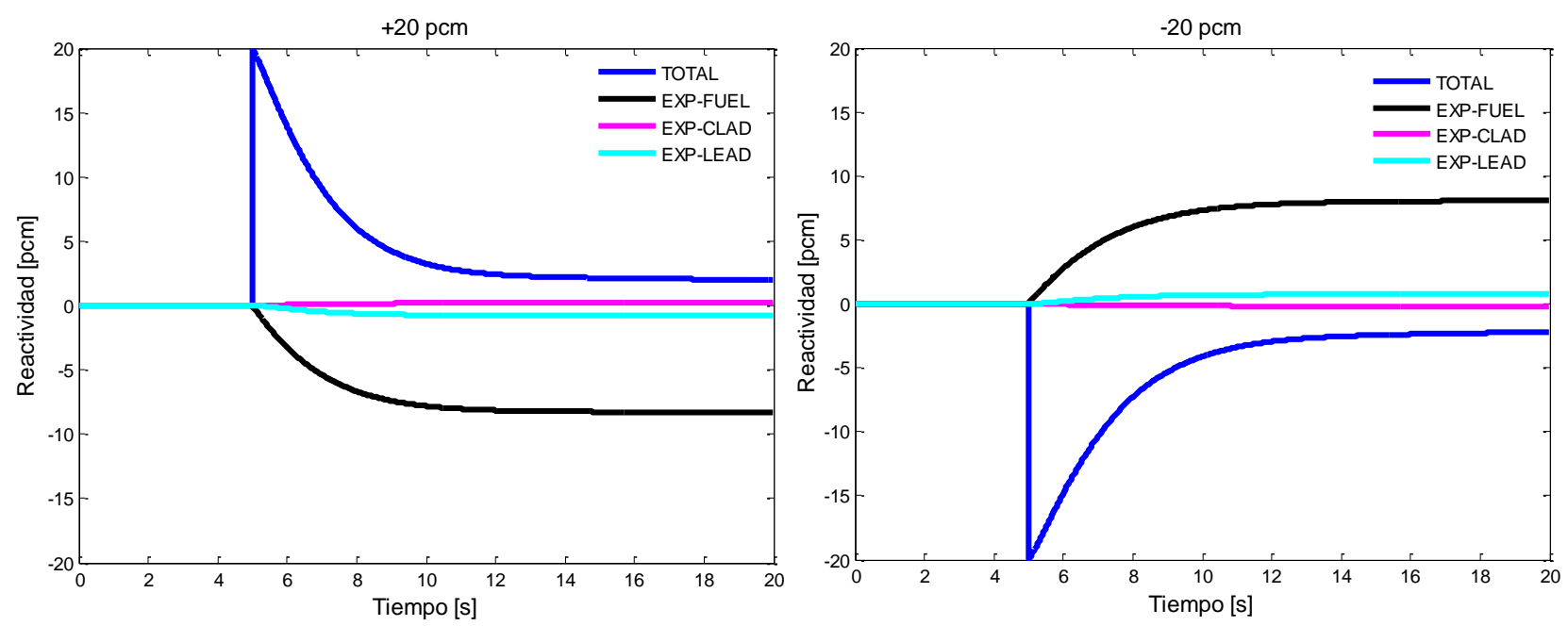

Figura 7.20. Reactividades en una perturbación de reactividad

Es interesante observar que en este transitorio, la reactividad por expansión del refrigerante no tiene un impacto tan grande como en los casos anteriores, debido a que en esos casos la perturbación fue directa en el plomo y en este caso la perturbación afecta inicialmente a los procesos neutrónicos.
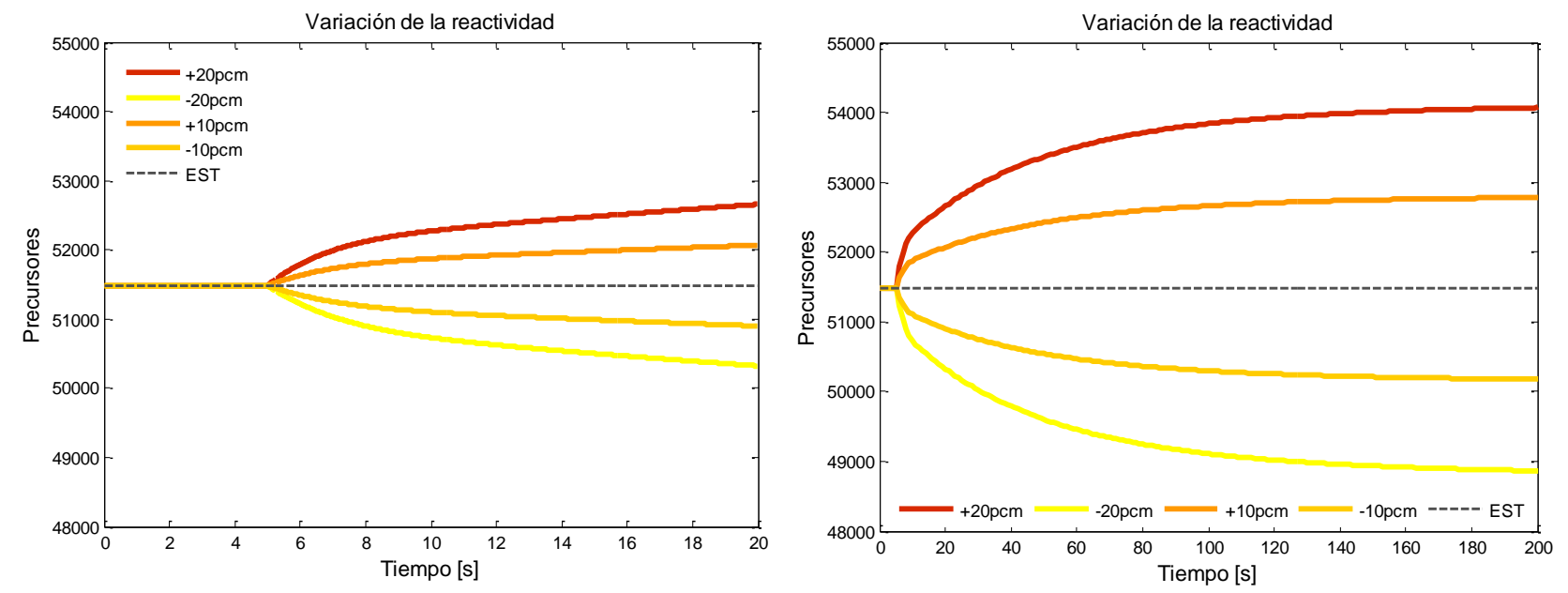

Figura 7.21. Concentración de precursores durante la perturbación de la reactividad.

En el caso de los precursores de neutrones, ocurre lo mismo que con la potencia, si aumenta la reactividad, éstos aumentan y en caso contrario, disminuyen. La concentración de precursores es importante porque contribuyen a la dinámica del reactor (Figura 7.21). 


\section{Análisis de sensibilidad e incertidumbre}

Un análisis de sensibilidad se relaciona con la cuantificación de los efectos en la solución óptima de los cambios en los parámetros del modelo, es decir, estimar la sensibilidad de los parámetros de salida del modelo al momento de modificar algún valor de entrada (Farías, 2000; ITESM, 2015).

El objetivo es cuantificar el efecto del cambio del flujo y temperatura de entrada del refrigerante en la potencia, la reactividad y la temperatura de salida del refrigerante.

Esta sección se divide en 4 partes principales

- Demostrar el estado estacionario

- Metodología de aplicación del Método Monte Carlo

- Aplicación de la metodología

- Resultados

\subsection{Estado estacionario}

La metodología aplicada para el análisis de sensibilidad solo es válida para condiciones de estado estacionario, entonces, para poder aplicar este método es necesario determinar el tiempo en que el modelo pasa de un estado transitorio a un estado estacionario. Para realizar lo anterior retomamos los resultados de la Sección 7.2, en donde se aplicó una perturbación al modelo para simular transitorios.

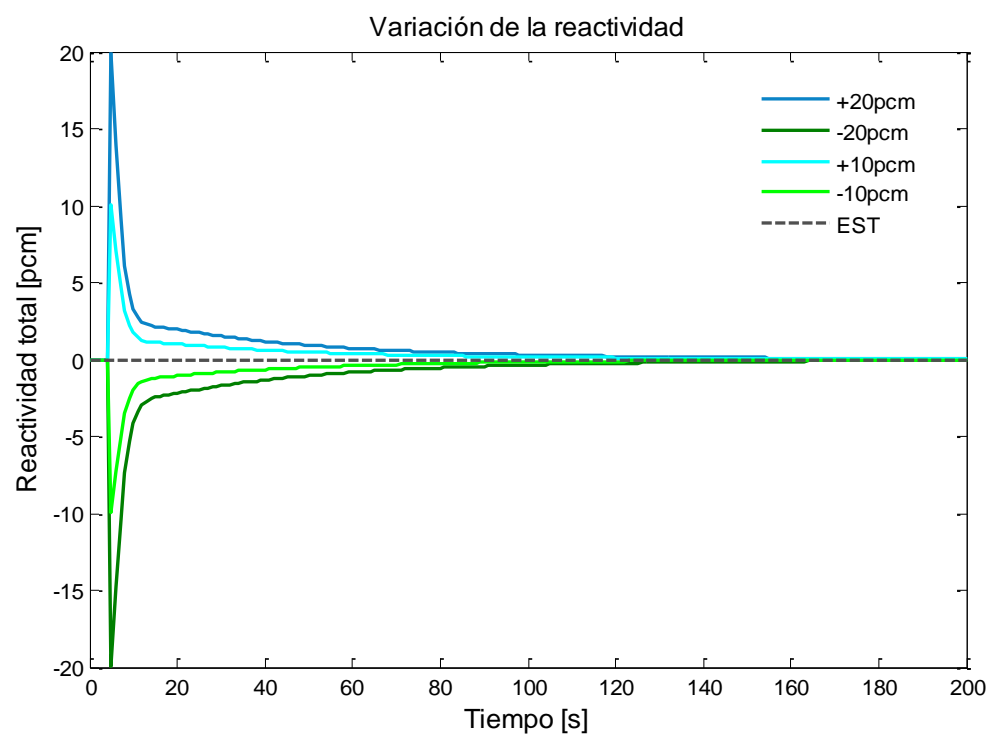

Figura 8.1.Variación de la reactividad para determinar el tiempo de estabilidad. 
En la Figura 8.1 se muestra la variación de la reactividad, la perturbación inicia a los 5 segundos, posteriormente el modelo regresa a la estabilidad. Al analizar Figura 8.1 se considera que es suficiente con 150 segundos para garantizar que el modelo dinámico alcanza un estado estacionario. Por lo tanto, para efectos de la aplicación del método Monte Carlo se tomarán $150 \mathrm{~s}$ para las simulaciones con la variación del parámetro de entrada.

\subsection{Metodología}

La metodología utilizada para el análisis de sensibilidad se describe a continuación (Quezada et al., 2016):

1. Generar los números pseudoaleatorios con una distribución uniforme $U(0,1)$

2. Conversión de los números aleatorios generados con distribución uniforme $U(0,1)$ a números aleatorios con distribución normal $N(0,1)$.

3. Selección de un valor de entrada y generar una muestra aleatoria de este valor a partir de la aplicación de los números de la distribución normal $N(0,1)$

4. Implementación de la muestra aleatoria del parámetro de entrada en el modelo numérico del reactor nuclear, con la finalidad de construir una distribución de los parámetros de salida seleccionados.

5. Realizar un análisis de invariabilidad utilizando la desviación estándar relativa (DER), para la determinación del tamaño de la muestra aleatoria para el análisis Monte Carlo.

6. Realizar el análisis de sensibilidad.

\subsection{Aplicación de la metodología}

\section{Generación de los números pseudoaleatorios}

El método Monte Carlo se basa en la utilización de números aleatorios, para la generación de estos números se deben buscar procedimientos algorítmicos computacionales que los generen de forma muy rápida y los puedan almacenar sin utilizar mucha capacidad de memoria (ITSTB, 2002), por lo tanto, en este trabajo se utilizaran números pseudoaleatorios.

Un número pseudoaleatorio es un número $U(0,1)$ producido por un algoritmo matemático. Las propiedades de los números pseudoaleatorios son (Triviño, 2016): 
- Uniformemente distribuidos, es decir, que los valores que se encuentran en el intervalo $(0,1)$ no se encuentre solo en una región, si no que se distribuyan por todo el espacio y no existe una recurrencia de aparición.

- Estadísticamente independientes, i.e., cuando el comportamiento estadístico de un número aleatorio no se ve afectado por los valores que toma otro número y no tengan una correlación serial.

- Reproducibles, es decir, cuando el método para generar los números comienza con la misma semilla, debe dar la misma secuencia de números pseudoaleatorios.

Para la generación de los números se utiliza el Método de Congruencia, el cual está dado por (Abramowitz \& Stegun, 1964):

$$
X_{n+1}=\left(a X_{n}+b\right)(\bmod T)
$$

La secuencia de números pseudoaleatorios se denota por $\left\{X_{n}\right\}$, con $n=1,2,3 \ldots, a, b$ y $T$ números primos y $\bmod T$ representa el módulo de $T$.

Para iniciar con la generación de los números pseudoaleatorios, es necesario tener una semilla $X_{0}$, el cual es el primer número aleatorio de la secuencia, no debe de ser cero y no debe de ser un número par.

Ya que se tiene la secuencia de números pseudoaleatorios es necesario que se distribuyan uniformemente en el espacio $U(0,1)$ para lo cual se utiliza la siguiente expresión:

$$
R_{n}=\frac{X_{n}}{T+1}
$$

Donde $R_{n}$ es el número pseudoaleatorio en el espacio $U(0,1)$ y $X_{n}$ es el número pseudoaleatorio generado previamente. Los valores empleados para la generación de números aleatorios son: $T=2^{31}-1$, $X_{0}=123457, a=7^{5}$ y $b=0$. Por lo tanto, el periodo $p$ es $2^{31}-2$.

Se generaron 2000 números pseudoaleatorios, en la Figura 8.2 se puede observar que la muestra se encuentra uniformemente distribuida en el espacio. 


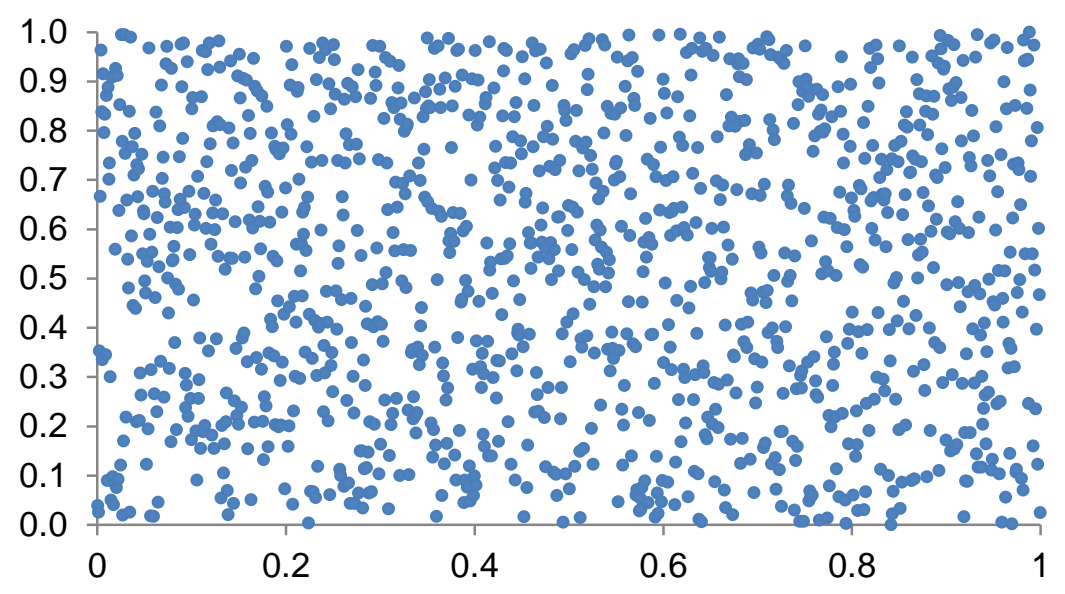

Figura 8.2. Números pseudoaleatorios distribuidos uniformemente en el espacio U(0,1).

\section{Conversión de los números aleatorios}

El siguiente paso es convertir los números de una distribución uniforme a una distribución normal $N(0,1)$. Para este paso se utiliza el método de Box-Muller (Kroese, 2011), el cual está basado en una transformación polar a partir de las ecuaciones:

$$
\begin{aligned}
& X_{1}=\sqrt{-2 \log \left(U_{1}\right)} \cos \left(2 \pi U_{2}\right) \\
& X_{2}=\sqrt{-2 \log \left(U_{1}\right)} \sin \left(2 \pi U_{2}\right)
\end{aligned}
$$

Con la aplicación de este método, se obtienen los valores $X_{1}$ y $X_{2}$, los cuales se distribuye de forma independiente en $N(0,1)$ (Gentle, 2003)

En la Figura 8.3 se muestra la distribución de los números pseudoaleatorios con una distribución normal, es decir, la mayor cantidad de los números se distribuyen cerca de cero, y conforme se alejan de cero la cantidad es menor.

En la Figura 8.4 se muestra la frecuencia de distribución de los números pseudoaleatorios, como se puede observar se tiene una distribución en la cual la mayoría de los números se encuentran en el centro y conforme los valores se alejan del centro la frecuencia disminuye. 


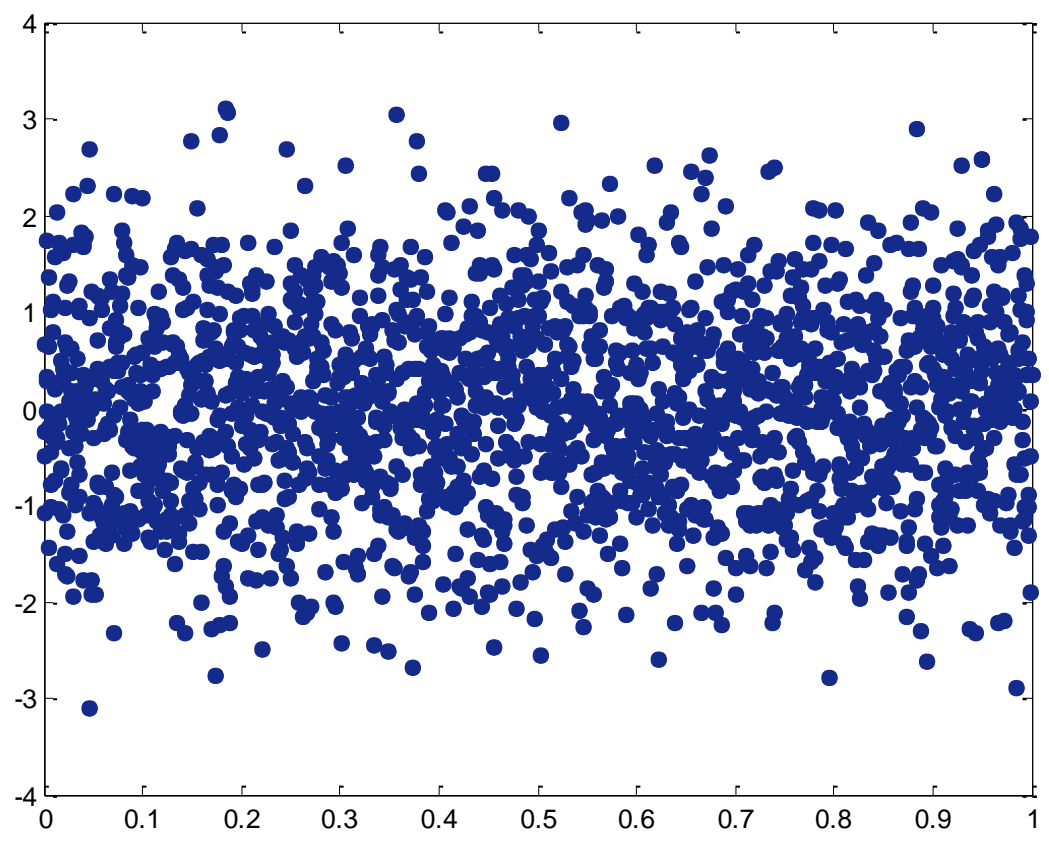

Figura 8.3. Números pseudoaleatorios con distribución normal $(M N)$.

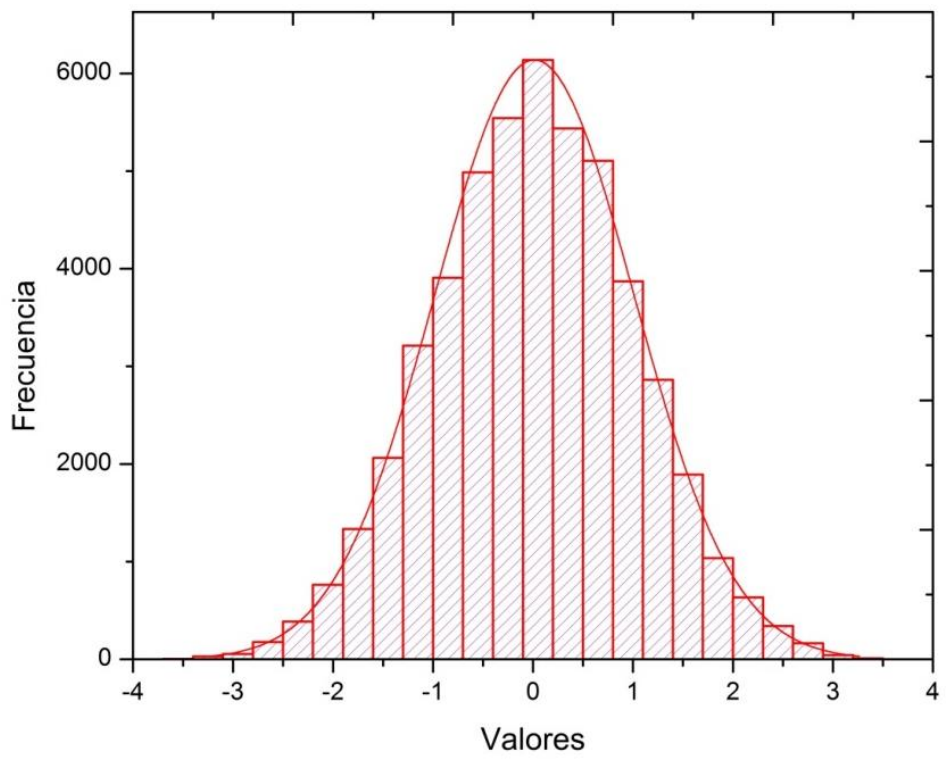

Figura 8.4. Frecuencia de los números pseudoaleatorios en una distribución normal. 


\section{Selección de un valor de entrada y aplicación de los números aleatorios}

Dentro del modelo del reactor rápido enfriado con plomo se tienen diferentes variables de entrada que pueden ser utilizadas dentro del método Montecarlo, en este caso se decidió utilizar el flujo de entrada del refrigerante $W_{i n}$ y la temperatura de entrada del refrigerante $T_{\text {leadin }}$, ya que estos parámetros son determinantes en el funcionamiento del reactor. A partir de la siguiente expresión se aplicará la muestra de números pseudoaleatorios al valor de flujo de entrada (Quezada et al., 2016)

$$
\gamma_{n}=\gamma_{0}\left(1+b M N_{n}\right)
$$

donde $\gamma_{0}$ es el valor inicial del parámetro de entrada, $b$ es la tasa de cambio que se desea aplicar, $M N_{n}$ es el número pseudoaleatorio y $\gamma_{n}$ es el parámetro de entrada generado. Para el flujo de entrada la ecuación 8.5 es de la forma

$$
W_{i n, n}=38430.0182\left(1+0.1 M N_{n}\right)
$$

Y para la temperatura de entrada de refrigerante es

$$
T_{l_{e a d_{i n}, n}}=673.15\left(1+0.025 M N_{n}\right)
$$

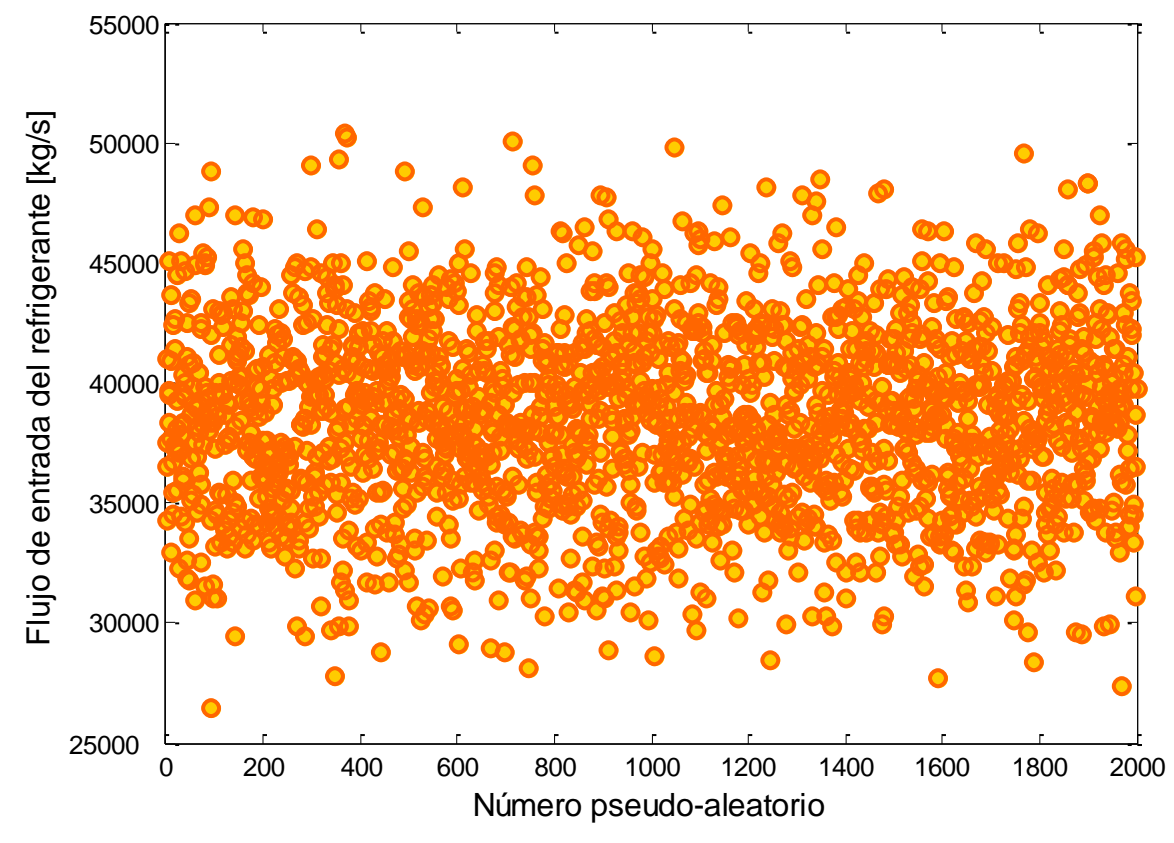

Figura 8.5. Distribución de los valores de flujo generados. 


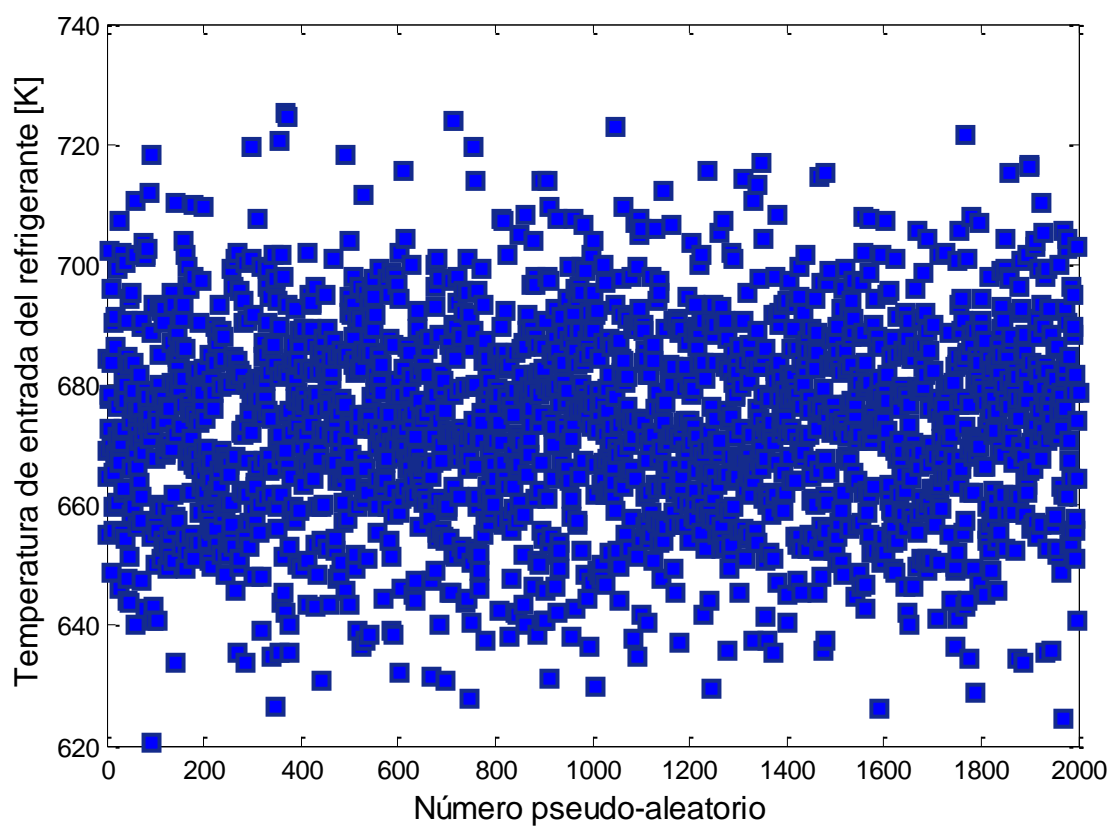

Figura 8.6. Distribución de los valores de temperatura generados.

La tasa de cambio aplicada al flujo es del $10 \%$ y de temperatura es de $2.5 \%$, esta última se seleccionó por las restricciones de temperatura del plomo, ya que su punto de fusión se da en $600 \mathrm{~K}$.

En la Figura 8.5 se muestra la distribución de los valores de flujo, estos valores van desde 30,169.42 kg/s a 46,300.18 kg/s, y en el caso de la temperatura de entrada del plomo los valores van desde $620.6 \mathrm{~K}$ a 725.2 K (Figura 8.6).

\section{Implementación}

La implementación del método Monte Carlo dentro del código consiste en dos bucles o lazos. El primero permite avanzar en los números aleatorios generados, i.e. hasta 2000, leyendo cada número y aplicándolo como variable de entrada y el segundo lazo cuenta el tiempo, en este caso 150 s.

Aplicando las ecuaciones 8.6 y 8.7 con cada número pseudoaleatorio generado se obtienen 2000 valores de flujo de entrada y 2000 de temperatura inicial del plomo, los cuales van a ser usados en el modelo del reactor para obtener la sensibilidad de las variables de salida. 


\section{Análisis de invariabilidad}

Determinar el tamaño de la muestra representativa es necesario para asegurar la invariabilidad de los resultados, este tamaño se determina utilizando la desviación estándar relativa (DER). Ese paso es de gran importancia para minimizar el tiempo de ejecución, ya que si se usa una muestra infinita de datos la teoría dice que la invariabilidad será nula, pero imposible de simular en cualquier computadora. Entonces con este procedimiento de la metodología establecemos con muy buena precisión el tamaño de la muestra.

El tamaño de la simulación óptimo se obtiene cuando la DER es independiente del tamaño de la muestra.

La DER está dada por (Canavos, 1988):

$$
D E R=\frac{s}{\bar{X}}(100)
$$

Donde $s$ es la desviación estándar y $\bar{X}$ es la media aritmética.

Aplicando la DER a los valores de flujo y temperatura de entrada de refrigerante se obtiene la Figura 8.7. Como se puede observar, para tamaños entre $10^{1}$ y $10^{2}$ los valores de las desviaciones son muy diferentes, es decir, hay variación en los resultados. Pero en tamaños de $10^{3}$ el valor de la DER alcanza cierta invariabilidad, para el flujo el $10 \%$ y para la temperatura el $2.5 \%$. Con este resultado podemos establecer que el tamaño de la muestra mayor a 1000 datos es suficiente para garantizar invariabilidad de la muestra y obtener resultados estadísticos confiables. En este trabajo aplicamos 2000 datos para el análisis de sensibilidad. 


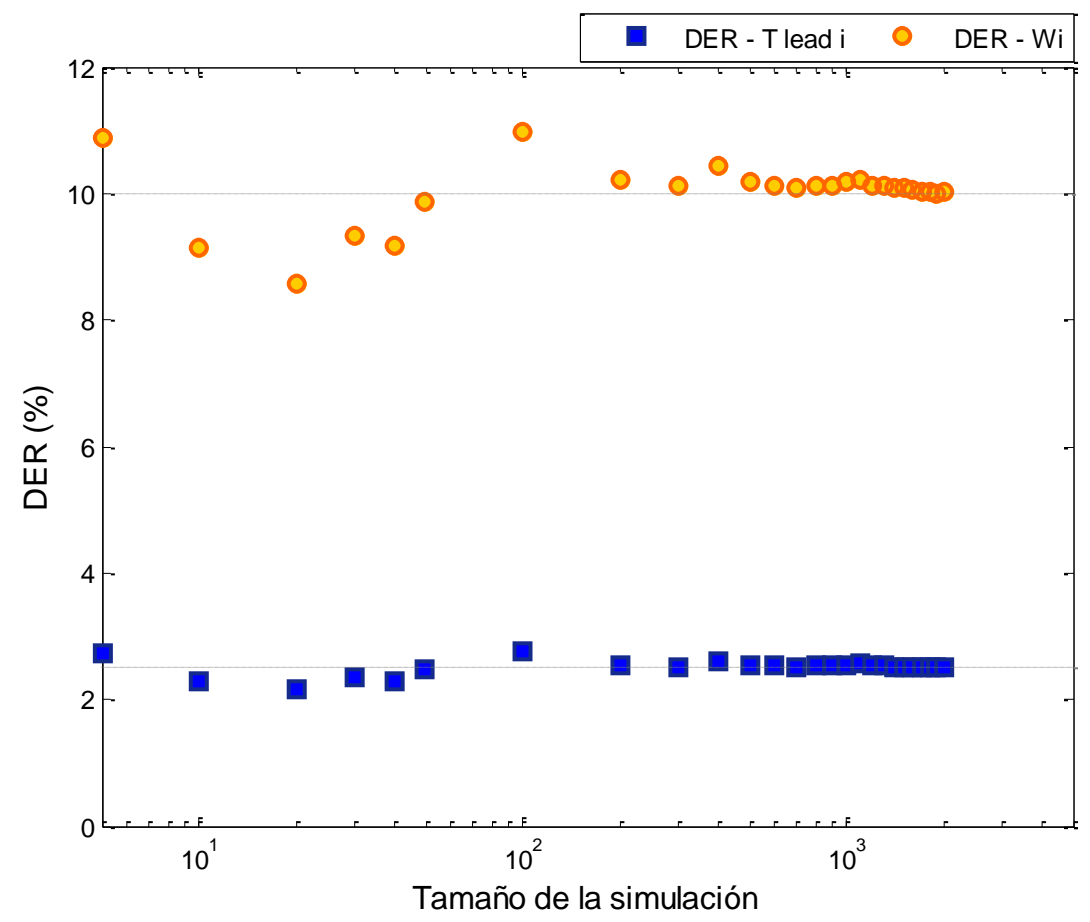

Figura 8.7. DER del flujo de entrada ( $\left.W_{\text {in }}\right)$ y de la temperatura de entrada del refrigerante $\left(T_{\text {lead }_{i n}}\right)$.

\section{Análisis de sensibilidad e incertidumbre}

Para realizar el análisis de sensibilidad es necesario realizar una simulación por cada valor de la variable generado, entonces, se tendrán 2000 simulaciones y cada simulación se correrá con 150 segundos, tiempo en que el reactor se estabiliza. Entonces como usamos un paso de integración de $10^{-4} \mathrm{~s}$, el código numérico se ejecutará $3 \times 10^{9}$ veces, es decir 3,000 millones de veces para cada variable. En total, para el flujo y la temperatura se ejecutará 6,000 millones de veces.

Los parámetros de salida monitoreados son la potencia $(P)$, la reactividad $(\rho)$, la temperatura de salida del refrigerante $\left(T_{\text {lead }}\right.$ out $)$ y la temperatura máxima de clad $\left(T_{\text {clad }_{\max }}\right)$.

A continuación, en las Figuras 8.8, 8.9 se muestran los valores de potencia, reactividad y temperatura obtenidos para los diferentes valores de flujo y temperatura de entrada respectivamente. Las líneas punteadas señalan el límite máximo y mínimo del intervalo.

Para los valores de las variables de salida obtenidas, se calculó su incertidumbre, para la cual se utilizó la ecuación de la incertidumbre estándar (Miranda, 2011; Manzur, 2010), la cual está dada por 


$$
u\left(x_{i}\right)=\sqrt{\frac{\sum_{k=1}^{n}\left(x_{i}-\bar{x}\right)^{2}}{n(n-1)}}
$$

Donde $x_{i}$ es el valor de obtenido de potencia, reactividad o temperatura, $u$ es la incertidumbre, $\bar{x}$ es el promedio aritmético de todos los valores y $n$ es el número de valores.

El intervalo generado entre el valor máximo y mínimo de los valores de la potencia, reactividad, temperatura de salida del refrigerante y temperatura máxima de pared, es la sensibilidad de la variable con respecto al parámetro de entrada al que se le aplicó el método de Monte Carlo.

La variación del 10\% en el flujo de entrada del refrigerante genera una respuesta de la potencia térmica de $11.6 \%$ con una incertidumbre de \pm 0.00042 , el cambio en la temperatura máxima del clad es de $100.78 \mathrm{~K}$ con una incertidumbre de \pm 0.24702 , la temperatura de salida del refrigerante tiene un cambio de 101.3629 $\mathrm{K}$ con una incertidumbre de \pm 0.0 .3641 y finalmente la reactividad tiene un cambio de $0.4394 \mathrm{pcm}$ con una incertidumbre de \pm 0.00142 . Estos resultados son resumidos en la Tabla 8.1.

Tabla 8.1. Valor máximo, mínimo e incertidumbre generados para la variación de flujo del refrigerante.

\begin{tabular}{cccc}
\hline Parámetro & Máximo valor generado & Mínimo valor generado & Incertidumbre \\
\hline $\begin{array}{c}\text { Potencia } \\
\text { [normalizada] } \\
\text { Reactividad } \\
\text { [pcm] }\end{array}$ & 1.0577 & 0.9418 & 0.00042 \\
$\begin{array}{c}\text { Temperatura de salida } \\
\text { del refrigerante [K] }\end{array}$ & 0.2001 & -0.2393 & 0.00142 \\
$\begin{array}{c}\text { Temperatura máxima } \\
\text { de clad [K] }\end{array}$ & 807.2531 & 705.8902 & 0.3641 \\
\hline
\end{tabular}

De forma similar, en la Tabla 8.2 se encuentran los cambios generados en las variables de salida pero con la variación de la temperatura de entrada del refrigerante. En este caso con la variación del $2.5 \%$ en el flujo de entrada del refrigerante se genera una respuesta de la potencia térmica de $4.01 \%$ con una incertidumbre de \pm 0.00013 , el cambio en la temperatura máxima del clad es de $65.23 \mathrm{~K}$ con una incertidumbre de \pm 0.36052 , la temperatura de salida del refrigerante tiene un cambio de $62.899 \mathrm{~K}$ con una incertidumbre de \pm 0.2389 y finalmente la reactividad tiene un cambio de 0.1478 pcm con una incertidumbre de \pm 0.00044 . Estos resultados son resumidos en la Tabla 8.2. 
Tabla 8.2. Valor máximo, mínimo e incertidumbre generados para la variación del temperatura de entrada del refrigerante.

\begin{tabular}{cccc}
\hline Parámetro & Máximo valor generado & Mínimo valor generado & Incertidumbre \\
\hline $\begin{array}{c}\text { Potencia } \\
\text { [normalizada] }\end{array}$ & 1.0131 & 0.9730 & 0.00013 \\
$\begin{array}{c}\text { Reactividad [pcm] } \\
\text { Temperatura de salida } \\
\text { del refrigerante[K] }\end{array}$ & 0.0669 & -0.0809 & 0.00044 \\
$\begin{array}{c}\text { Temperatura máxima } \\
\text { de pared [K] }\end{array}$ & 799.5462 & 736.6523 & 0.2389 \\
\hline
\end{tabular}
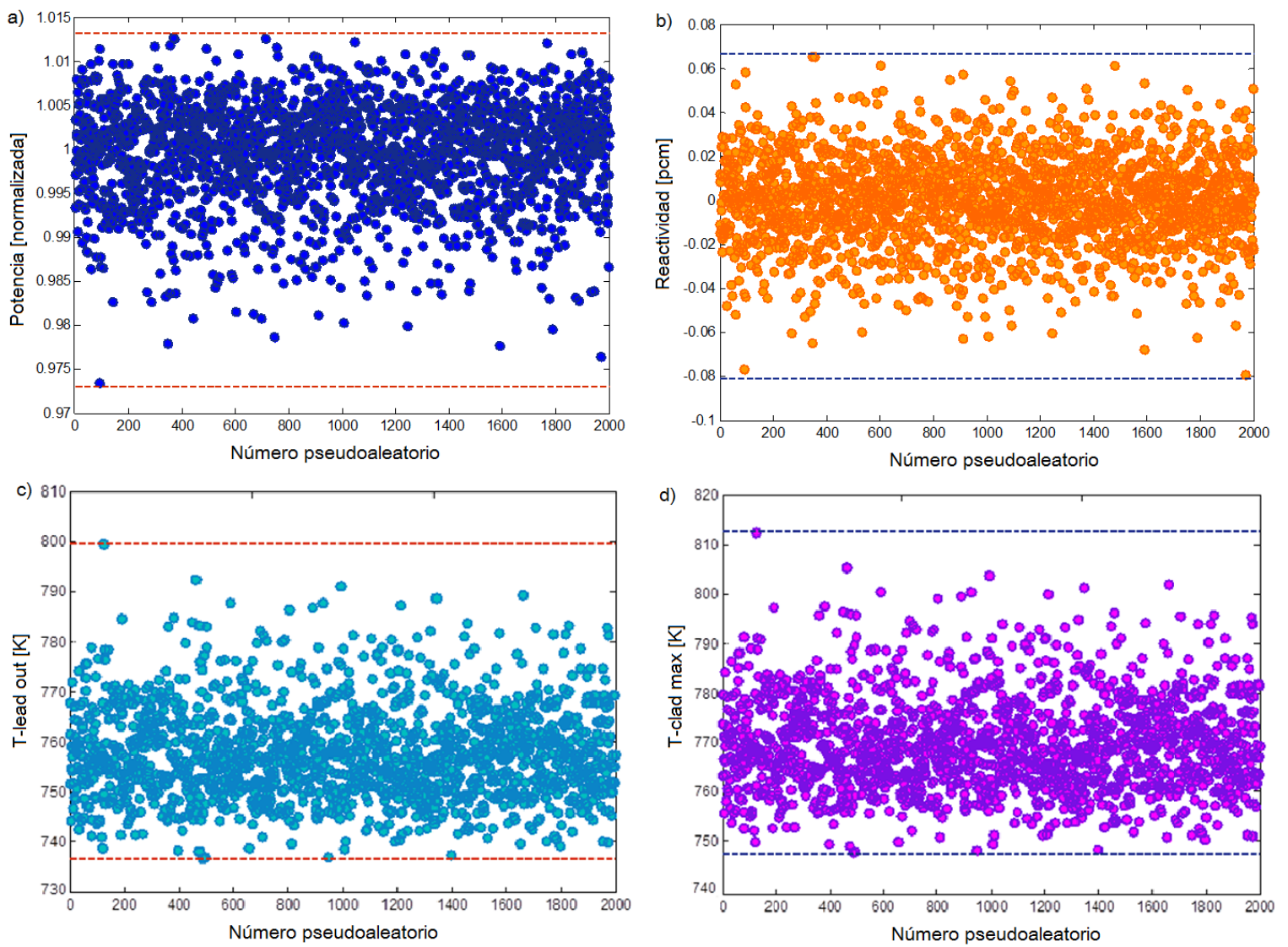

Figura 8.8. Distribución de los valores de a) potencia, b) reactividad, c) temperatura de entrada del refrigerante y d) temperatura máxima de clad generados con la variación del flujo. 

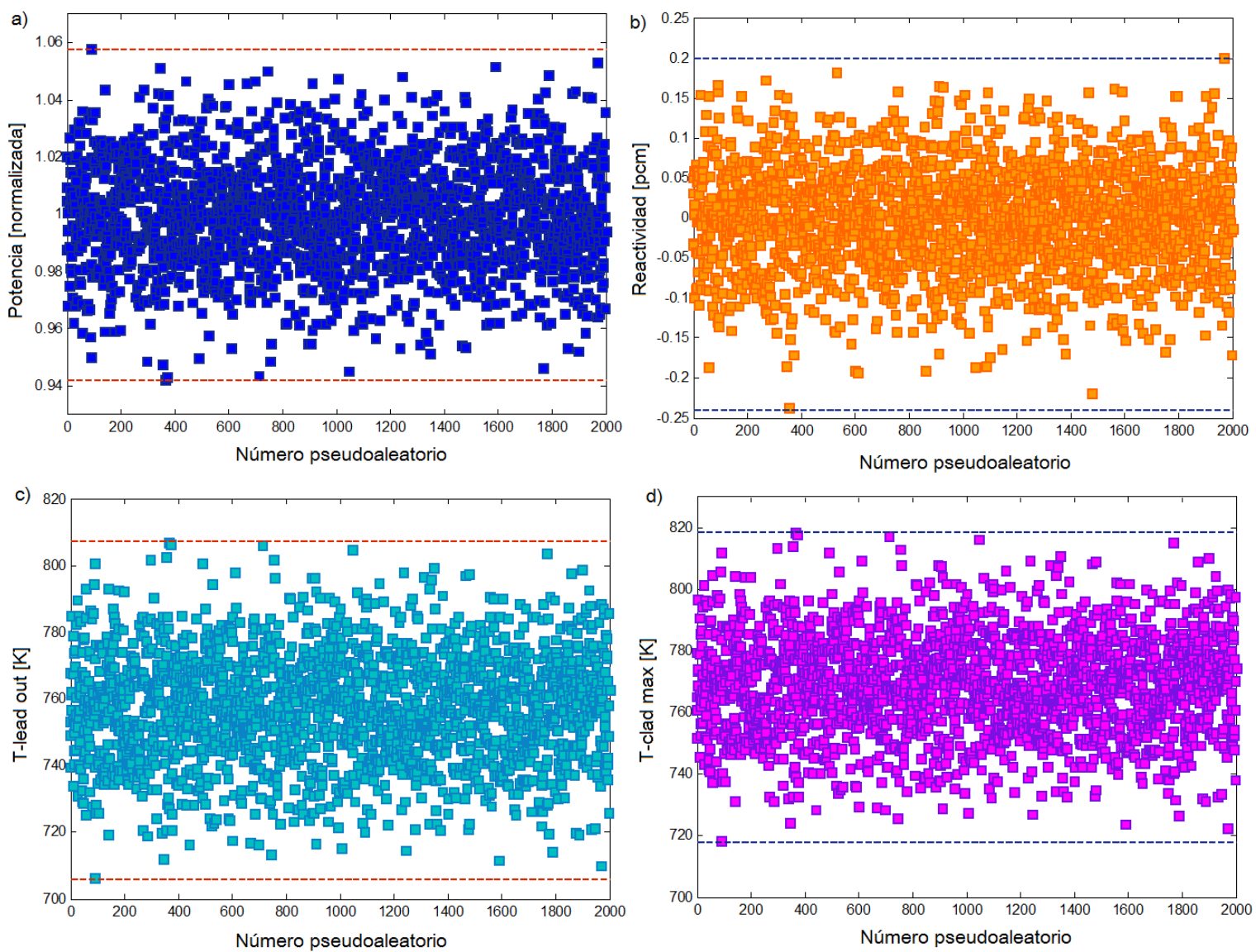

Figura 8.9. Distribución de los valores de a) potencia, b) reactividad, c) temperatura de entrada del refrigerante y d) temperatura máxima de clad generados con la variación de temperatura de entrada del refrigerante.

La incertidumbre de las variables de salida puede ser propagada mediante los resultados de la DER (Quezada et al., 2016), en las Figuras 8.10 y 8.11 se muestra la DER obtenida para la potencia, temperatura de salida del refrigerante y temperatura máxima del clad.

Para una variación del $10 \%$ en flujo de entrada de refrigerante, $P$ tuvo una variación de $0.58 \%, T_{\text {clad }}$ max de $1.90 \%$ y $_{\text {lead }_{\text {out }}}$ de $1.22 \%$.

Con una variación del $2.5 \%$ en temperatura de entrada del plomo, la $P$ tuvo un cambio de $1.87 \%$, $T_{\text {clad }_{\max }}$ de $2.10 \%$ y la variación de $T_{\text {lead }}$ out fue de $2.15 \%$. 


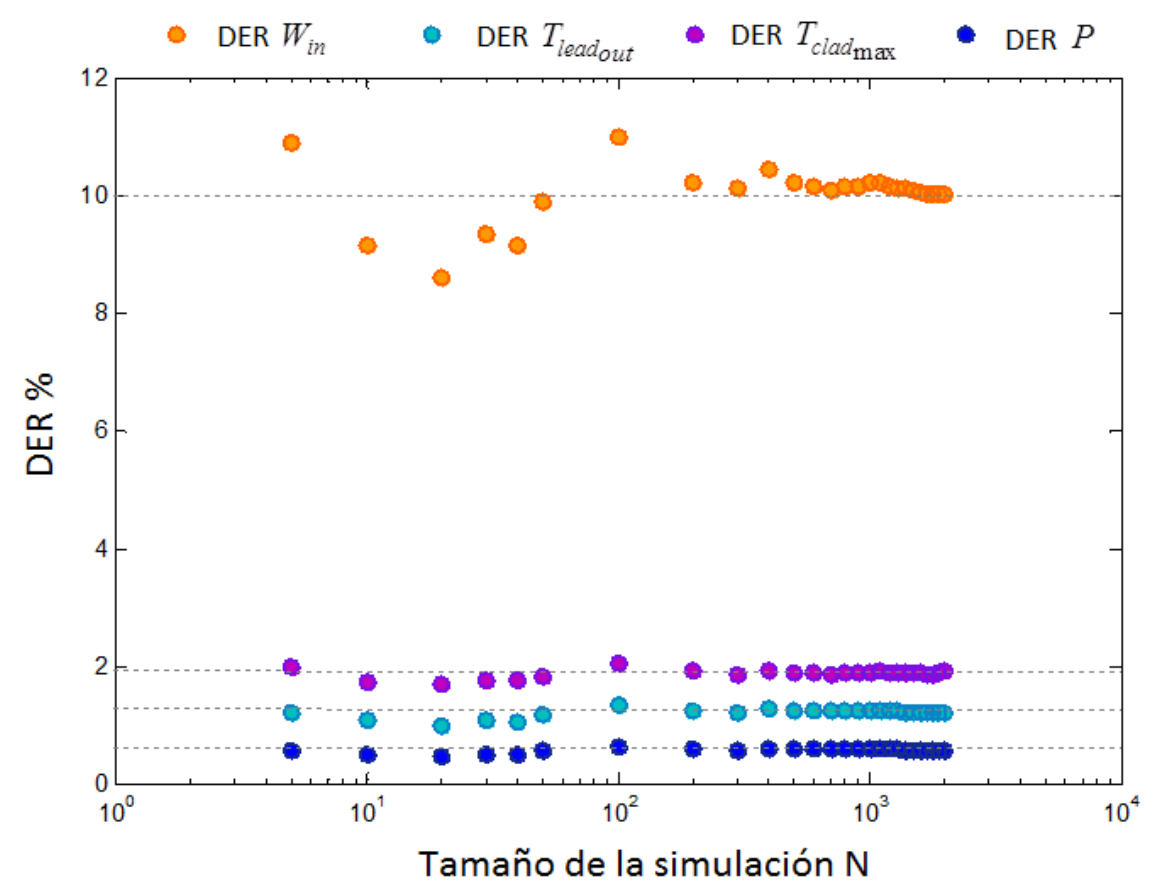

Figura 8.10. Desviación estándar relativa (DER) para $P, T_{\text {lead }_{\text {out }}}, T_{\text {clad }_{\max }}$ con variación de flujo de entrada.

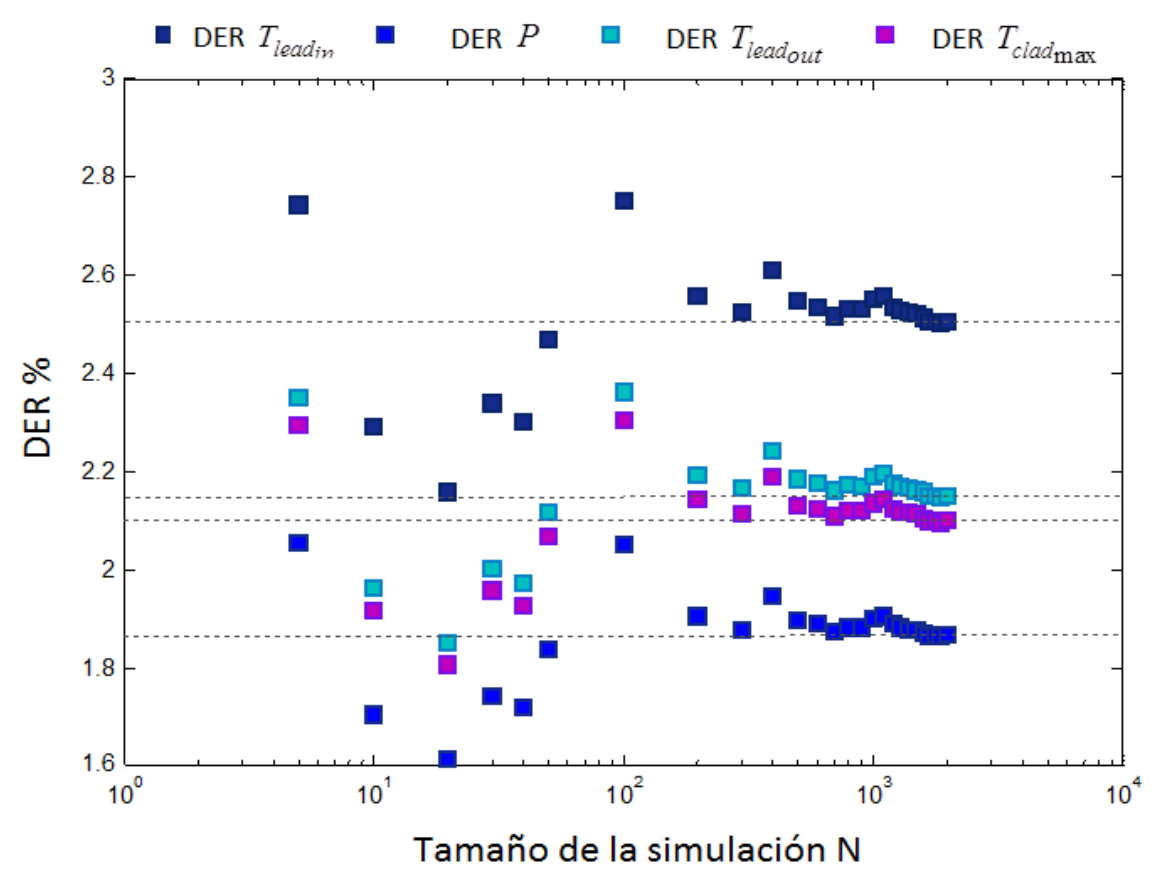

Figura 8.11. Desviación estándar relativa (DER) para $P, T_{\text {lead }_{\text {out }}}, T_{\text {clad }_{\max }}$ con variación de temperatura de entrada. 
Es importante conocer la relación entre los parámetros de entrada y de salida del sistema, esto se obtiene mediante una correlación estadística para obtener la ecuación que relaciona estas variables. Las Figuras 8.12 y 8.13 muestran la influencia del flujo de entrada y temperatura de entrada del refrigerante sobre la potencia, reactividad, temperatura de salida del plomo y temperatura máxima del clad.

Para el cambio de flujo, las mejores correlaciones son cubicas, por otro lado para la temperatura de entrada las correlaciones son lineales. En ambos casos $R^{2} \approx 1$.
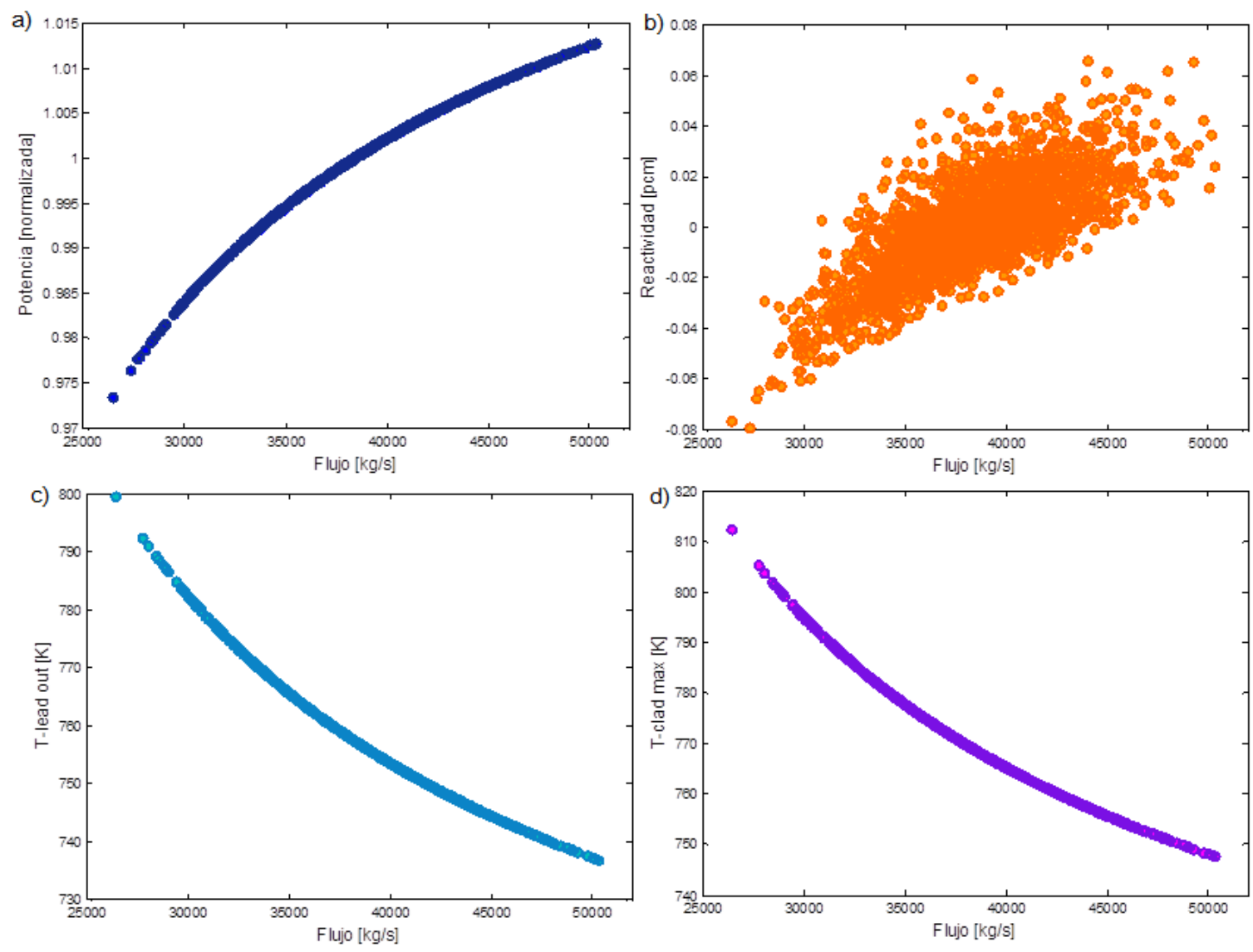

Figura 8.12. Efecto de la variación del flujo de refrigerante sobre: a) la potencia, b) la reactividad, c) temperatura de salida del refrigerante y d) temperatura máxima de clad.

En la Tabla 8.3 se muestran las correlaciones obtenidas así como los valores de $R^{2}$, el intervalo de confianza utilizado fue de $95 \%$. Con estas correlaciones es posible predecir las variables de salida del reactor modificando el flujo de entrada o la temperatura de entrada del refrigerante, lo cual puede ser aplicado en el diseño y operación de los Reactores enfriados con plomo. 

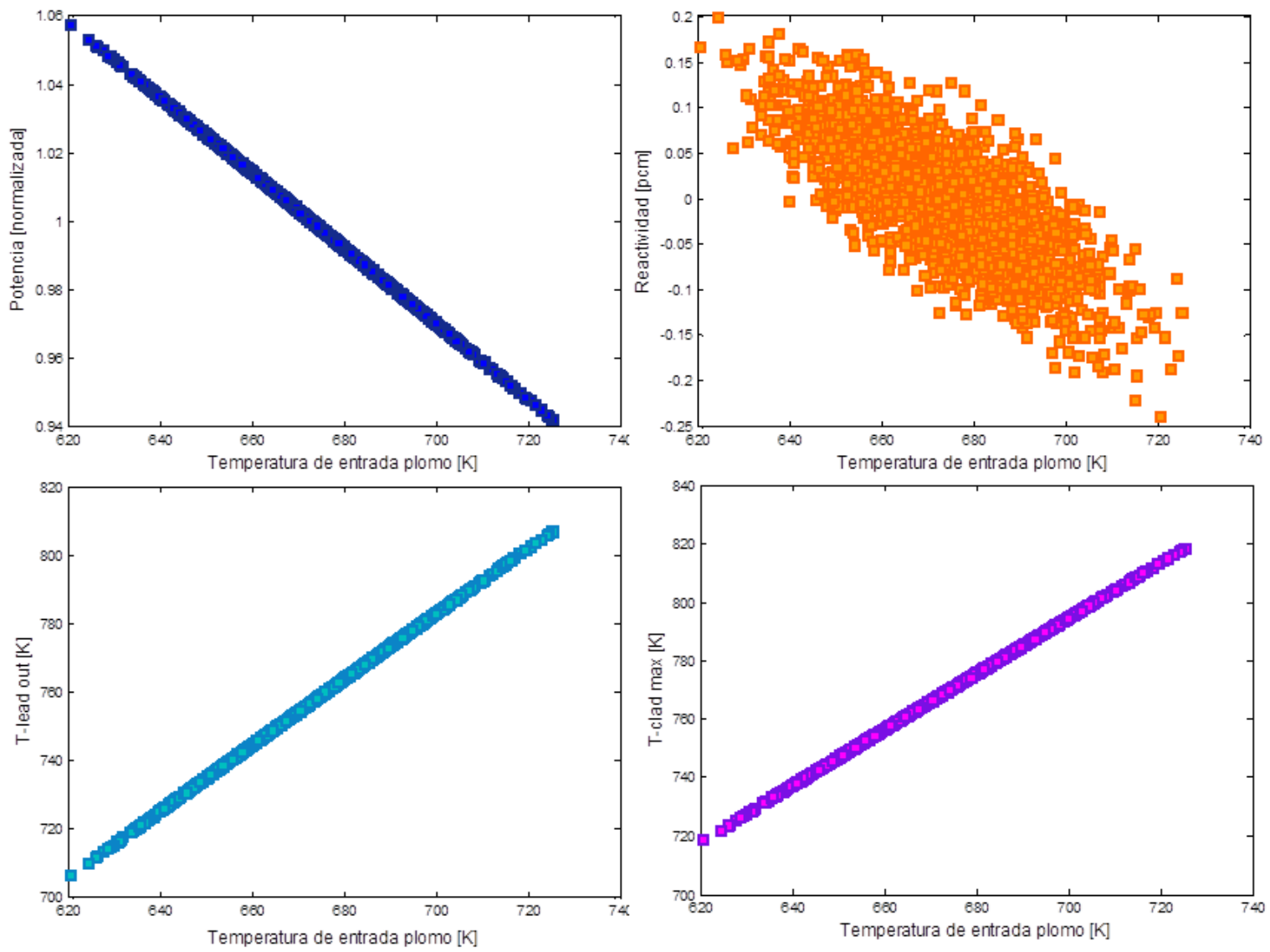

Figura 8.13. Efecto de la variación de la temperatura de entrada del refrigerante sobre: a) la potencia, b) la reactividad, c) temperatura de salida del refrigerante y d) temperatura máxima de clad. 
Tabla 8.3. Correlaciones entre las variables de entrada y las variables de salida, y valor de $R^{2}$

\begin{tabular}{|c|c|}
\hline Ecuación & $R^{2}$ \\
\hline $\begin{aligned} P= & \left(1.421 \times 10^{-15} \pm 9.112 \times 10^{-13}\right) W_{i n}{ }^{3}+\left(-2.0854 \times 10^{-10} \pm 1.0583 \times 10^{-12}\right) W_{i n}{ }^{2} \\
& +\left(1.1152 \times 10^{-5} \pm 4.0695 \times 10^{-8}\right) W_{i n}+0.7987 \pm 0.0005\end{aligned}$ & 0.999923 \\
\hline $\begin{array}{l}T_{\text {clad }_{\max }}=\left(-2.2206 \times 10^{-12} \pm 1.307 \times 10^{-14}\right) W_{i n}{ }^{3}+\left(3.2817 \times 10^{-7} \pm 1.5207 \times 10^{-9}\right) W_{i n}{ }^{2} \\
\quad+\left(-0.0177 \pm 5.8577 \times 10^{-5}\right) W_{i n}+1091.9653 \pm 0.7469\end{array}$ & 0.999956 \\
\hline $\begin{array}{l}T_{\text {lead }}{ }_{\text {out }}=\left(-2.2275 \times 10^{-12} \pm 1.364 \times 10^{-14}\right) W_{\text {in }}^{3}+\left(3.2839 \times 10^{-7} \pm 1.5318 \times 10^{-9}\right) W_{\text {in }}{ }^{2} \\
\quad+\left(-0.0176 \pm 5.9005 \times 10^{-5}\right) W_{\text {in }}+1076.5272 \pm 0.7523\end{array}$ & 0.999952 \\
\hline$P=\left(-0.0011 \pm 1.3656 \times 10^{-7}\right) T_{\text {lead }}+1.7440 \pm 9.1965 \times 10^{-5}$ & 0.999969 \\
\hline$T_{\text {lead }_{\text {out }}}=\left(0.9566 \pm 7.5253 \times 10^{-5}\right) T_{\text {lead }_{\text {in }}}+124.9653 \pm 0.0507$ & 0.999987 \\
\hline$T_{\text {lead }_{\text {out }}}=\left(0.9645 \pm 6.8388 \times 10^{-5}\right) T_{\text {lead }_{\text {in }}}+107.7904 \pm 0.0460$ & 0.999989 \\
\hline
\end{tabular}




\section{Conclusiones}

El propósito de esta tesis de maestría fue incursionar en el modelado matemático y numérico de reactores de cuarta generación. Específicamente se describieron los reactores nucleares enfriados con plomo (LFR), los cuales forman parte de los reactores enfriados por líquidos metálicos de Generación IV.

Se estudió y analizó el núcleo del reactor ALFRED de $300 \mathrm{MW}_{\text {th }}$, para ello y por estrategias de modelado matemático se dividió en tres principales procesos:

- Procesos neutrónicos de generación de potencia

- Difusión de calor en el combustible

- Procesos de flujo de fluidos y transferencia de calor en el refrigerante plomo

Cada uno de ellos se modeló independientemente y se identificaron las variables de interacción entre estos procesos (Fig. 6.2). El modelado es totalmente transitorio y unidimensional, excepto el proceso neutrónico que es puntual. Las principales conclusiones del trabajo se presentan a continuación:

1. Se realizaron experimentos numéricos en estado estacionario (Sección 7.1), obteniendo resultados de temperaturas dentro de los márgenes establecidos en el diseño de ALFRED. Estas temperaturas son importantes para la seguridad en el funcionamiento del reactor.

- La temperatura máxima del combustible fue de $1720.06 \mathrm{~K}$, la cual se mantiene debajo del límite de diseño, la cual es de $2273.15 \mathrm{~K}$.

- La temperatura máxima del encamisado obtenida es de $749.43 \mathrm{~K}$, es decir, $73.72 \mathrm{~K}$ menor al límite de diseño.

- La temperatura de salida del refrigerante fue de $753 \mathrm{~K}$, la cual es igual al límite establecido en ALFRED.

2. En estado transitorio se realizaron pruebas a $100 \%$ de potencia y con perturbaciones en la temperatura de entrada del refrigerante velocidad de entrada de refrigerante y reactividad por barras de control. (Sección 7.2).

- Las pruebas en estado transitorio muestran eventos simulados de pérdida de refrigerante, por falla de los calentadores del refrigerante y la inserción y extracción de barras de control, mostrando el comportamiento seguro de la potencia del reactor. 
- A partir del inicio del estado transitorio, el sistema regresa a un estado estacionario después de 150s (en reactores típicos es un orden de magnitud menor)

- Durante la perturbación de temperatura, la temperatura promedio máxima del combustible fue de $1264.85 \mathrm{~K}$, del encamisado $752 \mathrm{~K}$ y de salida del refrigerante de $736.85 \mathrm{~K}$, obtenidas cuando la temperatura de entrada del plomo es de $693 \mathrm{~K}$.

- Con temperatura del refrigerante de $693 \mathrm{~K}$, la potencia tuvo una disminución de $2 \%$ al llegar al estado estacionario y con un aumento de reactividad de $2.3 \mathrm{pcm}$, en donde la contribución mayor negativa fue por efecto Doppler y positiva por expansión del refrigerante.

- Para la perturbación de velocidad se obtuvieron las temperaturas más altas con $1 \mathrm{~m} / \mathrm{s}$, para el combustible es de $1290.48 \mathrm{~K}, 800.72 \mathrm{~K}$ para el encamisado y $783.85 \mathrm{~K}$ para la salida del plomo refrigerante.

- La potencia, cuando la velocidad es de $1 \mathrm{~m} / \mathrm{s}$, es de 0.93 y la reactividad es de -7 pcm, en donde la mayor contribución es negativa por expansión del refrigerante.

- En la perturbación de reactividad, con un aumento de $20 \mathrm{pcm}$, la potencia aumento 10\%. La temperatura del combustible es de $1293 \mathrm{~K}$, del clad es de $736.91 \mathrm{~K}$ y del refrigerante de $720.46 \mathrm{~K}$.

3. Los principales resultados obtenidos de aplicar el método de Monte Carlo, para realizar el análisis de sensibilidad e incertidumbre (Sección 8), en el flujo de entrada de refrigerante y la temperatura del refrigerante de entrada son:

- Con las correlaciones es posible predecir los valores de salida, y aplicarlo a operación y diseño de ALFRED.

- El tamaño de simulación óptimo $\mathrm{N}$ obtenido a partir de la desviación estándar relativa (DER) fue de 1000, para este trabajo se utilizó una muestra de 2000 números aleatorios (Fig. 8.7).

- Para calcular la incertidumbre, el caudal de entrada del refrigerante se varió un 10\% y la temperatura de entrada del refrigerante $2.5 \%$, en estos rangos se generaron los valores de entrada.

- La aplicación de los valores de entrada por Monte Carlo al modelo ALFRED genera los intervalos de sensibilidad de los parámetros de las variables de salida (potencia del reactor, temperatura máxima del revestimiento, temperatura de salida del refrigerante y reactividad total).

- Se obtuvieron correlaciones entre los parámetros de entrada (flujo y temperatura de entrada del refrigerante) y los de salida (potencia, temperatura de salida del refrigerante, temperatura máxima del clad) (Fig. 8.12-13) que pueden ser aplicadas para diseño y análisis de reactores LFR. 
Los resultados y modelos desarrollados en esta tesis permitieron someter dos artículos en revistas internacionales de prestigio en el área nuclear. Se participó en un congreso nacional organizado por la sociedad nuclear mexicana (SNM). También a través del proyecto AZTLAN platform se realizó una estancia en la escuela de verano "Frédéric Joliot \& Otto Hahn" en Aix-en-Provence, Francia, con el fin de incrementar los conocimientos sobre reactores rápidos. 


\section{Recomendaciones o Perspectivas}

Con base en los resultados obtenidos en el trabajo, se realizan las siguientes recomendaciones:

- Realizar un modelo del termofluido con más de una dimensión, es decir, trabajar en direcciones $r, z, \theta$, manteniendo la dependencia con el tiempo.

- Incluir un modelo neutrónico tridimensional.

- Realizar un análisis de 2 y de 3 canales promedio de acuerdo con la zona de enriquecimiento del núcleo.

- Realizar el análisis para describir todos los procesos y geometría de la vasija del reactor.

- Explorar los procesos de convección natural del refrigerante.

- Realizar el análisis de incertidumbre en estado transitorio. 


\section{Nomenclatura}

\section{Símbolos latinos}

$A_{f} \quad$ área de flujo $\left[\mathrm{m}^{2}\right]$

$C_{i} \quad$ densidad del grupo $i$ de precursores $\left[\mathrm{cm}^{-3}\right]$

Cp calor específico $\left[\mathrm{J} \mathrm{kg}^{-1} \mathrm{~K}^{-1}\right]$

$d_{\text {rod }}$ diámetro de barra de combustible [m]

$D_{h} \quad$ diámetro equivalente $[\mathrm{m}]$

$G \quad$ flujo másico $\left[\mathrm{kg} \mathrm{m}^{-2}\right]$

$h \quad$ coeficiente de transferencia de calor $\left[\mathrm{W} \mathrm{m}^{-2} \mathrm{~K}^{-1}\right.$ ]

$k \quad$ conductividad térmica $\left[\mathrm{W} \mathrm{m} \mathrm{m}^{-1} \mathrm{~K}^{-1}\right]$

$K_{D}$ constante Doopler [pcm]

$l_{p} \quad$ pitch de barra [m]

$M N_{n} \quad$ número Monte Carlo[-]

$n \quad$ densidad neutrónica $\left[\mathrm{cm}^{-3}\right]$

$N$ tamaño de simulación [-]

$\mathrm{Nu}$ número de Nusselt [-]

$P \quad$ potencia térmica en el sub canal [W]

$P_{o} \quad$ potencia térmica nominal [W]

$P_{m} \quad$ perímetro mojado [m]

Pr número de Prandtl [-]

$q^{\prime \prime \prime} \quad$ fuente de calor $\left[\mathrm{W} \mathrm{m}^{-3}\right]$

$r \quad$ radio $[\mathrm{m}]$

Re número de Reynolds [-]

$R_{n} \quad$ número pseudo aleatorio en $U[1,0]$ 
DER desviación estándar relativa [\%]

$s \quad$ desviación estándar [-]

$t \quad$ tiempo [s]

$T \quad$ temperatura $[\mathrm{K}]$

$\left\langle T_{\text {fuel }}\right\rangle$ temperatura promedio del combustible [K]

$\left\langle T_{f u e l}\right\rangle_{0}$ temperatura de referencia del combustible [K]

$\left\langle T_{\text {clad }}\right\rangle$ temperatura promedio del clad [K]

$\left\langle T_{\text {clad }}\right\rangle_{0}$ temperatura de referencia del clad [K]

$\left\langle T_{\text {lead }}\right\rangle$ temperatura promedio del plomo [K]

$\left\langle T_{\text {lead }}\right\rangle_{0}$ temperatura de referencia del plomo [K]

$T_{\text {lead }}$ temperatura de entrada del refrigerante $[\mathrm{K}]$

$T_{\text {lead }_{\text {out }}}$ temperatura de salida del refrigerante [K]

$V_{f} \quad$ volumen del combustible $\left[\mathrm{m}^{-3}\right]$

$W_{\text {in }} \quad$ flujo de entrada del refrigerante $\left[\mathrm{kg} \mathrm{s}^{-1}\right]$

$W_{\text {out }}$ flujo de salida del refrigerante $\left[\mathrm{kg} \mathrm{s}^{-1}\right]$

$X_{n} \quad$ número pseudo aleatorio

$\bar{X} \quad$ promedio aritmético

\section{Símbolos griegos}

$\alpha_{c} \quad$ coeficiente de reactividad por expansión del clad [pcm/K]

$\alpha_{f}$ coeficiente de reactividad por expansión del combustble [pcm/K]

$\alpha_{l} \quad$ coeficiente de reactividad por expansión del plomo $[\mathrm{pcm} / \mathrm{K}]$

$\alpha_{t h} \quad$ coeficiente de expansión térmica 
$\beta \quad$ fracción de neutrones retardados [-]

$\Lambda \quad$ tiempo de generación de neutrones [s]

$\lambda \quad$ constante de decaimiento $\left[\mathrm{s}^{-1}\right]$

$\mu_{\text {lead }} \quad$ viscosidad [Pa s]

$\xi_{f r} \quad$ coeficiente de fricción [-]

$\rho \quad$ reactividad $[\mathrm{pcm}]$

$\rho_{0} \quad$ reactividad inicial $[\mathrm{pcm}]$

$\Gamma \quad$ densidad $\left[\mathrm{k} \mathrm{gm}^{-3}\right]$

$\gamma_{n} \quad$ valor inicial generado

$\gamma_{0} \quad$ valor de la variable de salida

$\psi \quad$ distribución de potencia axial 


\section{Referencia Bibliográfica}

Alemberti, A. (2015a). ELFR Main Systems and Components. Education Trainig Seminar on Fast Reactors Science and Technology. ITESM México. 20 de junio a 02 de julio.

Alemberti, A. (2015b). ALFRED. Education Trainig Seminar on Fast Reactors Science and Technology. ITESM México. 20 de junio a 02 de julio.

Artioli, C., Grasso, G., Petrovich, C. (2010). A new paradigm for core design aimed at the sustaintaility of nuclear energy: The solution of the extended equilibrium state. Annals of Nuclear Energy, 37. 915-922.

Aufiero, M. C. (2013). A Multi-physics time-dependent model for the Lead Fast Ractor single-channel analysis. Nuclear Engineering and Design, 256, 14-27.

Avramova, M., Ivanov, K. (2007). Challenges in couplen thermal-hydraulicls and neutronics simulations for LWR safety analysis. Annals of Nuclear Energy, 34, 501-5013.

Bandini, G., Bubelis, E., Schikorr, M., Alemberti, A., Mansani, L. (2015). Analysis of Unprotected Transients in the Lead-Cooled ALFRED Reactor. Education Trainig Seminar on Fast Reactors Science and Technology. ITESM México. 20 de junio a 02 de julio.

Barragán, A. (2013). Diseño neutrónico y termohidráulico de un reactor nuclear enfriado con agua supercrítica. Tesis de doctorado. UNAM.

Bird, R., Steward, W., Lightfoot, E. (2014). Fenómenos de transporte. Reverté.

Bortot, S., Moisseytsev, A., Sieniki, J., Artioli, C. (2011). Core design investigation for a SUPERSTAR small modular lead-cooled fast reactor demonstrator. Nuclear Engineering and Desing, 30213031.

Caccia, F. (2014). Diseño conceptual de un reactor rápido. Tesis de Licenciatura. Univesidad Nacional de Cuyo.

Campos R., E. E., Osornio S., B., Espinosa P., G. (07 de 2015). Propiedades Termofísicas de metales líquidos: Plomo. Reporte final de Servicio Social. UAM-Iztapalapa.

Canavos, G. C. (1988). Probabilidad y Estadística. México: Mc Graw Hill. 
Cinotti, L., Smith, C., Sekimoto, H., Mansani, L., Reale, M., Sienicki, J.(2011). Lead-cooled System design and challenges in the frame of Generation IV International Forum. Journal of Nuclear Materials, 415, 245-253.

Carbajo, J., Yoder, G., Popov, S., Ivanov, V. (2001). A review of the thermophysical properties of MOX and UO2 fuels. Journal of Nuclear Materials, 181-198.

Courcelle, A. ( 2016). LMFR Clads and Structural Materials. The 2016 Frédéric Joliot \& Otto Hahn Summer School. Aix-en-Provence, Francia.

Espinosa-Paredes, G. and Espinosa-Martínez, E.-G. (2009). Fuel rod model based on Non-Fourier heat conduction equation. Annals of Nuclear Energy 36, 680-693.

Espinosa-Paredes, G., Vázquez, R. (2016). Física de Rectores nucleares I. Un enfoque conceptual. OmniaScience. Primera Edición.

Farías, E. B. (2000). Análisis de Sensibilidad. Ediciones del Instituto Politécnico Nacional. http://users.dcc.uchile.cl/ nbaloian/DSS-DCC/Esp/3_5.pdf. Consultado en octubre de 2016.

Gentle, J. (2003). Statistics and computing, Random number generation and Monte Carlo Methods. Springer.

Giannetti, F., Di Maio, D. V., Naviglio, A., \& Caruso, G. (2016). Thermal-hydraulic analysis of an innovative decay heat removal system for lead-cooled fast reactors. Nuclear Engineering and Design, 305, 168-178.

GIF (2002). A Technology Roadmap for Generation IV Nuclear Energy Systems. U.S. DOE Nuclear Energy Research Advisory Committe and the Generation IV International Forum.

GIF. (2009). GIF R\&D Outlook for Generation IV Nuclear Energy Systems. Generation IV International Forum.

GIF. (2016). Generation IV International Forum. https://www.gen-4.org/gif/jcms/c_9260/Public. Consultado en febrero de 2016.

Glassone, S., Sesonske, A. (1990). Ingeniería de reactores nucleares. Reverté

González-Romero, E. (2012). Reactores de Generación IV: Camino a la sostenibilidad a largo plazo de la energía nuclear. Publicaciones DYNA. Energía y Sostenibilidad. España. 
Grasso, G. (2016). State-of-the-art Reactor Phyycs Methods for LMFR Core design with Illustrations taken from ASTRID/SFR and ELFR/LFR Projects. The 2016 Frédéric JOLIOT \& Otto HAHN Summer School. Aix-en-Provence, Francia.

Grasso, G., Mikityuk, K., Petrovich, C., Mattioli, D., Manni, F., Gugiu, D. (2013). Demonstrating the effectiveness of the European LFR concept: The ALFRED core design. IAEA publicaciones.

Grasso, G., Petrovich, C., Mattioli, D., Artioli, C., Sciora, P., Gugiu, D.(2014). The core design of ALFRED, a demonstrator for the European lead-cooled reactors. Nuclear Engineering and Design, 287-301.

IAEA. (2012). Fast reactors and related fuel cycles: Challenges and opportunites (FR09). International Atomic Energy Agency, Viena.

IAEA. (2014). Examen de la Tecnología Nuclear-2014.

IEA. (2015). International Energy Agency, Monthly electricity statistics. http://www.iea.org/statistics/monthlystatistics/monthlyelectricitystatistics. Consultado en marzo de 2016.

IAEA (2016). International Atomic Energy Agency. Nuclear technology and applications/Energy. https://www.iaea.org/topics/energy. Consultado en febrero de 2016.

IAEA (2017). International Atomic Energy Agency. PRIS. Power Reactor Information System. https://www.iaea.org/pris/. Consultado en febrero de 2017.

ININ. (2014). Secretaría de Energía - Instituto Nacional de Investigaciones Nucleares. http://www.inin.gob.mx/temasdeinteres/fisionnuclear.cfm. Consultado en marzo de 2016.

ITESM. (2015). CCIR/Matemáticas. http://www.mty.itesm.mx/dmti/materias/tc3001/lecturas/tc3001-08intro-sensibilidad.pdf. Consultado el octubre de 2016.

ITSTB. (2002).http://simulacion-itstb.blogspot.mx/p/unidad-dos-numeros-aleatorios-y.html. Consultado en octubre de 2016.

Kroese, D. (2011). Handbook of Monte Carlo methods. Wiley.

Lodi, F., Grasso, G., Mattioli, D., \& Sumini, M. (2016). ANTEO+: A subchannel code for thermalhydraulic analysis of liquid metal cooled systems. Nuclear Engineering and Design, 301, 128152. 
Lopez, R. (2010). Diseño neutrónico de un reactor nuclear rápido de onda viajera. Tesis de maestría. UNAM,

Manzur, A. (2010). Universidad Autónoma Metropolitana Unidad Iztapalapa. Departamento de física. http://www.izt.uam.mx/newpage/contactos/anterior/n76ne/analisis_grafico2.pdf. Consultado en septiembre de 2016.

Miranda, J. (2011). UNAM. Facultad de Química. http://depa.fquim.unam.mx/amyd/archivero/eval_incert_11208.pdf. Consultado en septiembre de 2016.

Moshkbar-Bakhshayesh, K., B. Ghofrani, M. (2013). Transient identification in nuclear power plant: A review. Progress in Nuclear Energy, 23-32.

Patankar, S.V. (1980). Numerical Heat Transfer and Fluid Flow. McGraw-Hill.

Ponciroli, R., Bigoni, A., Cammi, A., Lorenzi, S., Luzzi, L. (2014a). Object-oriented modelling and smulation for the ALFRED dynamics. Progress in Nuclear Energy, 71, 15-29.

Ponciroli, R., Cammi, A., Lorenzi, S., Luzzi, L. (2014b). A preiminary approach to the ALFRED reactor control strategy. Progress in Nuclear Energy, 73, 113-128.

Ponciroli, R., Cammi, A., Lorenzi, S., \& Luzzi, L. (2016). Petri-net based modelling approach for ALFRED reactor operation and control system design. Progress in Nuclear Energy, 87, 54-66.

Quezada-García, S., Espinosa-Martínez, E., Espinosa-Paredes, G., Vázquez-Rodríquez, A. (2016). Powerfeedwater temperature operating domain for BWR driven by natral convection. Progress in Nuclear Energy, 86, 110-119.

Roelofs, F. (2016). Core Physics and Thermal-Hydraulics. Advanced Thermal-Hydraulics for LiquidMetal Reactors. The 2016 Frédéric JOLIOT \& Otto HAHN Summer School. Aix-en-Provence, Francia.

Smith, Craig. (2010). Lead-Cooled Fast Reactor (LFR) Design: Safety, Neutronics, Thermal Hydraulics, Structural Mechanics, Fuel, Core, and Plant Design. A compenium of Reactor Technology. Lawrencw Livermore National Laboratory. Estados Unidos de América.

Sobolev, V. Malambu, E., Aït Abderrahim, H. (2009). Design of a fuel element for a lead-cooled fast reactor. Journal of Nuclear Materials, 385, 392-399. 
Sobolev, V. (2011). Database of thermophysical properties of liquid metal coolants for GEN-IV. Sodium, lead, lead-bismut eutectic (and bismut). Scientific report of the Belgian Nuclear Research Centre.

Takahashi, M. (2015). Recent Activities and Development of GIF-LFR-SSC. The 23rd International Conference on Nuclear Engineering(ICONE 23) Japan

Todreas, N. E., Kazimi, M. (2012). Nuclear Systems, Thermal Hydraulic Fundamentals. CRC Press. Segunda edición.

Triviño, J. E. (2016). Generadores de Números aleatorios. Universidad Nacional de Colombia. http://disi.unal.edu.co/profesores/jeortizt/Sim/Archivos/03A.\%20GeneradoresDeNumerosAleator ios.pdf. Consultado en octubre de 2016.

United States Nuclear Regulatory Commission. (2016). United States Nuclear Regulatory Commission. http://www.nrc.gov/reading-rm/basic-ref/glossary/transient.html. Consultado en octubre de 2016.

Van Rooijen, W. F. G. (2016). Core Physics and Thermal-Hydraulics. Fundamentals of Fast Reactor Core and Fuel Cycle Physics. The 2016 Frédéric JOLIOT \& Otto HAHN Summer School. Aixen-Provence, Francia.

Waltar, A., Todd, D., Tsvetkov, P. (2012). Fast Spectrum Reactors. Springer.

Whitaker, S. (1983). Fundamental Principles of Heat Transfer. Krieger Publishing Company.

Eldén, L., Wittmeyer-Kock, L., Brunn, H., 2009. Introduction to Numerical Computation.Student Litterature. Sweden. 